\title{
OPTIMIZATION RESULTS FOR THE HIGHER EIGENVALUES OF THE $p$-LAPLACIAN ASSOCIATED WITH SIGN-CHANGING CAPACITARY MEASURES
}

\author{
MARCO DEGIOVANNI AND DARIO MAZZOLENI
}

\begin{abstract}
In this paper we prove the existence of an optimal set for the minimization of the $k$-th variational eigenvalue of the $p$-Laplacian among $p$-quasi open sets of fixed measure included in a box of finite measure. An analogous existence result is obtained for eigenvalues of the $p$-Laplacian associated with Schrödinger potentials. In order to deal with these nonlinear shape optimization problems, we develop a general approach which allows to treat the continuous dependence of the eigenvalues of the $p$-Laplacian associated with sign-changing capacitary measures under $\gamma$-convergence.
\end{abstract}

\section{Contents}

1. Introduction

2. Notations and preliminaries

3. Convergence of functionals and of inf-sup values $\quad 6$

$\begin{array}{lr}\text { 4. Nonlinear eigenvalue problems } & 14\end{array}$

5. Convergence of measures and of functionals $\quad 17$

$\begin{array}{ll}\text { 5.1. Convergence of capacitary measures } & 17\end{array}$

5.2. Lower estimate and asymptotic equicoercivity for a sequence of functionals 21

$\begin{array}{lr}\text { 5.3. Convergence of functionals } & 23\end{array}$

6. Towards variational eigenvalues for sign-changing capacitary measures 27

7. Semicontinuity properties of inf-sup values of measures 31

$\begin{array}{ll}\text { 7.1. Lower semicontinuity of inf-sup values of measures } & 31\end{array}$

7.2. Upper semicontinuity of inf-sup values of measures $\quad 34$

8. Existence of nonlinear eigenvectors for sign-changing capacitary measures 35

9. On the existence of optimal capacitary measures 37

$\begin{array}{lr}\text { References } & 44\end{array}$

\section{INTRODUCTION}

In the last few years, shape optimization problems for the eigenvalues of the Dirichlet-Laplacian have been a very studied topic in many fields of mathematics, see [31] for a general overview. Recently, there has been an interest in extending these results also to the case of the eigenvalues of the $p$-Laplacian for $p \neq 2$ (often called nonlinear eigenvalues). Given an open subset $\Omega$ of $\mathbb{R}^{N}$ with finite measure and $1<p<\infty$, we say that $\lambda>0$ is an eigenvalue of the $p$-Laplacian if there is a nonzero weak solution $u$, called eigenvector, of the problem

$$
\begin{cases}-\operatorname{div}\left(|\nabla u|^{p-2} \nabla u\right)=\lambda|u|^{p-2} u & \text { in } \Omega \\ u=0 & \text { on } \partial \Omega .\end{cases}
$$

2010 Mathematics Subject Classification. 49Q10, 47J10.

Key words and phrases. Shape optimization, variational methods, quasilinear elliptic equations, nonlinear eigenvalue problems.

The authors are members of the Gruppo Nazionale per l'Analisi Matematica, la Probabilità e le loro Applicazioni (GNAMPA) of the Istituto Nazionale di Alta Matematica (INdAM). They have been partially supported by the 2019 INdAM-GNAMPA project "Ottimizzazione spettrale non lineare". 
The eigenvalues can be characterized as the critical values of the functional

$$
f: W_{0}^{1, p}(\Omega) \rightarrow \mathbb{R}, \quad f(u)=\int_{\Omega}|\nabla u|^{p} d \mathcal{L}^{N},
$$

on the manifold $M=\left\{u \in W_{0}^{1, p}(\Omega): \int_{\Omega}|u|^{p} d \mathcal{L}^{N}=1\right\}$. The first eigenvalue can be proved to be a minimum, while higher eigenvalues (if $p \neq 2$ ) are less understood. More precisely, one can obtain a nondecreasing sequence of eigenvalues by the minimax procedure

$$
\lambda_{m}^{p}(\Omega)=\inf _{K \in \mathcal{K}_{m}} \sup _{u \in K} f(u) \quad \text { for all integer } m \geq 1
$$

where $\mathcal{K}_{m}$ denotes the collection of compact and symmetric subsets $K$ of $M$ such that $i(K) \geq m$ and $i$ denotes a suitable index, e.g. Krasnosel'skii genus, (see [28]). Unfortunately, it is still a major open problem to understand if all the eigenvalues of the $p$-Laplacian are of this form. In the present paper we focus on the "variational" eigenvalues arising from the minimax procedure described above.

A first shape optimization result for these eigenvalues was recently obtained by Fusco, Mukherjee and Zhang in [27, Theorem 1.2].

Theorem 1.1 (Fusco-Mukherjee-Zhang). Let $1<p<\infty, \Omega$ be a bounded and open subset of $\mathbb{R}^{N}$, $\left.c \in] 0, \mathcal{L}^{N}(\Omega)\right]$ and $F: \mathbb{R}^{2} \rightarrow \mathbb{R}$ be a function nondecreasing in each variable and lower semicontinuous.

Then the problem

$$
\min \left\{F\left(\lambda_{1}^{p}(A), \lambda_{2}^{p}(A)\right): A \text { is a p-quasi open subset of } \Omega \text { with } \mathcal{L}^{N}(A)=c\right\}
$$

admits a solution.

We note that, also when $A$ is only a $p$-quasi open set, it is possible to define the space $W_{0}^{1, p}(A)$ and then the variational eigenvalues $\lambda_{m}^{p}(A)$ again by (1.1)

The main aim of this paper is to extend this existence result also to higher nonlinear variational eigenvalues and to nonlinear eigenvalues associated with Schrödinger potentials. Actually, we follow a unified approach based on the concept of $p$-capacitary measure. The reason for which the above existence result was proved only for the first two eigenvalues is that a lower semicontinuity result for nonlinear eigenvalues with respect to an appropriate convergence was not known, as the typical arguments, relating convergence of quasi open sets and linear operators (see e.g. [9, Chapter 6]), cannot be adapted to the case $p \neq 2$. Let us collect the key results (see [27, Corollary 4.5 and Proposition 4.6]) involved in the proof of Theorem 1.1 .

Theorem 1.2 (Fusco-Mukherjee-Zhang). Let $\Omega$ be a bounded and open subset of $\mathbb{R}^{N}$ and $\left(A_{n}\right)$ be a sequence of p-quasi open sets $\gamma$-converging to a p-quasi open set $A$ in $\Omega$.

Then we have

$$
\lambda_{m}^{p}(A) \leq \liminf _{n \rightarrow \infty} \lambda_{m}^{p}\left(A_{n}\right) \quad \text { for } m=1,2 .
$$

In particular, the technique used in [27] in order to show the desired lower semicontinuity for $m=2$ is based on a mountain pass characterization of the second eigenvalue of the $p$-Laplacian on $p$-quasi open sets, and extending this approach to the case $m \geq 3$ seems out of reach. Thus, a key issue is to understand the continuity properties of the higher nonlinear variational eigenvalues with respect to the $\gamma$-convergence of $p$-quasi open sets.

In this paper we investigate in depth this question, developing the more general framework of $p$ capacitary measures, in which a lower semicontinuity result for nonlinear eigenvalues under $\gamma$-convergence is proved, see Proposition 9.1. Then, in Theorems 9.2 and 9.3, we provide some related optimization results, still in the setting of $p$-capacitary measures.

In the special case of $p$-quasi open sets, we deduce that Theorem 1.2 holds for all $m \geq 1$, see Corollary 9.5. As a consequence of Theorem 9.3, we can prove the following extension of Theorem 1.1 to higher nonlinear variational eigenvalues. This result provides also a theoretical setting for performing numerical simulations, as recently done in [3].

Theorem 1.3. Let $k \geq 1,1<p<\infty, \Omega$ be an open subset of $\mathbb{R}^{N}$ with finite measure, $\left.\left.c \in\right] 0, \mathcal{L}^{N}(\Omega)\right]$ and $F: \mathbb{R}^{k} \rightarrow \mathbb{R}$ be a function nondecreasing in each variable and lower semicontinuous. 
Then the problem

$$
\min \left\{F\left(\lambda_{1}^{p}(A), \ldots, \lambda_{k}^{p}(A)\right): A \text { is a p-quasi open subset of } \Omega \text { with } \mathcal{L}^{N}(A)=c\right\}
$$

admits a solution.

We now briefly describe the motivations for working in a class wider than $p$-quasi open sets and the other new results that we obtain. First of all, the works from the 1980s and 1990s of Buttazzo, Dal Maso, Mosco, Murat [11, 17, 19, 20] suggest that the natural setting for spectral problems in the line of (1.3) is the space of $p$-capacitary measures, i.e. Borel measures in $\Omega$ that vanish on sets of zero $p$-capacity. One can consider $\lambda$ to be an eigenvalue associated with the $p$-capacitary measure $\mu$ if there is a nonzero solution $u$ of the problem

$$
\begin{cases}-\Delta_{p} u+|u|^{p-2} u \mu=\lambda|u|^{p-2} u & \text { in } \Omega, \\ u=0 & \text { on } \partial \Omega,\end{cases}
$$

where the formal writing above should be read through the variational formulation described in [20]. On the other hand, also on the right hand side of the eigenvalue equation (1.4) things can become more complicated and more interesting. In particular, the study of eigenvalues with an $L^{\infty}$ sign-changing weight on the right hand side arises naturally in many problems from population dynamics (see [13] for an overview) and the existence of eigenvalues of the $p$-Laplacian was studied in [39] in the sign-changing case. We generalize also this sign-changing weight on the right hand side to be the difference of two non-negative $p$-capacitary measures and we set the problem in the whole $\mathbb{R}^{N}$ (with some additional assumptions on the measures). Summing all up, given three (non-negative) $p$-capacitary measures $\mu, \nu_{1}, \nu_{2}$, we study the variational eigenvalues $\lambda_{m}^{p}\left(\mu, \nu_{1}, \nu_{2}\right)$ of the problem

$$
\left\{\begin{array}{l}
-\Delta_{p} u+|u|^{p-2} u \mu=\lambda|u|^{p-2} u\left(\nu_{1}-\nu_{2}\right) \quad \text { in } \mathbb{R}^{N}, \\
\int|u|^{p} d \nu_{2}<\int|u|^{p} d \nu_{1},
\end{array}\right.
$$

with a homogeneous Dirichlet-type condition at infinity, noting that, in order to set the problem in a bounded and open subset $\Omega$ of $\mathbb{R}^{N}$, it is enough to replace $\mu$ with $\infty_{\mathbb{R}^{N} \backslash \Omega}+\mu$. The motivation for considering the case of $\mathbb{R}^{N}$ as ambient space is in view of a possible extension of the existence Theorem 1.3 for nonlinear spectral functionals to the case $\Omega=\mathbb{R}^{N}$, which is a difficult open problem that we plan to investigate in the future and that has been only recently solved in the case $p=2$ (see $[8,10,36]$ ).

Thanks to the general theory developed, we can also prove an extension to nonlinear eigenvalues of [12, Theorem 4.1] which deals, in the case $p=2$, with the optimization of Schrödinger potentials, that is, of capacitary measures absolutely continuous with respect to the Lebesgue measure in $\mathbb{R}^{N}$.

Theorem 1.4. Let $k \geq 1,1<p<\infty, \Omega$ be an open subset of $\mathbb{R}^{N}$ with finite measure, $\nu$ be a p-capacitary measure in $\Omega, \Psi:[0,+\infty] \rightarrow[0,+\infty]$ be a strictly decreasing and continuous function such that there exists $\alpha>1$ with $s \mapsto \Psi^{-1}\left(s^{\alpha}\right)$ convex on $\left\{s \geq 0: s^{\alpha} \in \Psi([0,+\infty])\right\}$,

$$
0<c \leq \Psi(0) \mathcal{L}^{N}(\Omega)
$$

and $F: \mathbb{R}^{k} \rightarrow \mathbb{R}$ be a function nondecreasing in each variable and lower semicontinuous. Denote by $\mathcal{V}$ the set of $\mathcal{L}^{N}$-measurable functions $V: \Omega \rightarrow[0,+\infty]$ such that

$$
\int_{\Omega} \Psi(V) d \mathcal{L}^{N} \leq c
$$

and such that there exists $u \in W_{0}^{1, p}(\Omega)$ satisfying

$$
\int|u|^{p} V d \mathcal{L}^{N}<+\infty, \quad \int|u|^{p} d \nu<\int|u|^{p} d \mathcal{L}^{N} .
$$

If $\mathcal{V} \neq \emptyset$, then there exists a minimizer $V$ for the problem

$$
\min \left\{F\left(\lambda_{1}^{p}(V), \ldots, \lambda_{k}^{p}(V)\right): V \in \mathcal{V}\right\}
$$

satisfying

$$
\int_{\Omega} \Psi(V) d \mathcal{L}^{N}=c
$$


where $\lambda_{m}^{p}(V)$ is associated with

$$
\begin{cases}-\Delta_{p} u+V|u|^{p-2} u=\lambda|u|^{p-2} u-\lambda|u|^{p-2} u \nu & \text { in } \Omega, \\ u=0 & \text { on } \partial \Omega, \\ \int_{\Omega}|u|^{p} d \nu<\int_{\Omega}|u|^{p} d \mathcal{L}^{N}, & \end{cases}
$$

according to Section 8 .

The most interesting examples of the function $\Psi$ for which the assumptions of the above theorem hold are $\Psi(s)=e^{-\beta s}$ for all $\beta>0$ and $\Psi(s)=s^{-\beta}$ for all $\beta>0$.

The abstract theory developed in this paper allows us also to prove an upper semicontinuity result for nonlinear eigenvalues of $p$-capacitary measures, under very mild assumptions, Theorem 7.4. Though this is not needed for the shape optimization problem that was our motivation, we believe it is a very important property and it involves an interesting reduction to finite dimensional spaces in the inf-sup procedure. Moreover, it could be useful when dealing with spectral problems with non-monotone functional.

The paper is organized as follows. After the Introduction and Section 2, where we recall the main notions of $p$-capacity, $p$-quasi open set, $p$-fine topology and $\Gamma$-convergence, the paper is divided into an abstract and an applied part.

The abstract part is developed in Sections 3 and 4, where first we study the behavior of sup functionals and of inf-sup values in a topological vector space and we prove suitable lower and upper semicontinuity results under $\Gamma$-convergence, then we study nonlinear eigenvalue problems involving a sign-changing weight in a reflexive Banach space.

The applied part of the paper is organized as follows. Section 5 is devoted to the study first of convergence properties of $p$-capacitary measures and then of convergence of related functionals in $L_{l o c}^{p}\left(\mathbb{R}^{N}\right)$, in the line of [19]. In Section 6 we define, in the $L_{l o c}^{p}\left(\mathbb{R}^{N}\right)$ setting, the variational eigenvalues involving sign-changing $p$-capacitary measures, we provide general conditions for existence of a sequence of (finite) variational eigenvalues and we provide an inf-sup characterization by means of suitable finite dimensional spaces. Section 7 is devoted to the study, still in the $L_{l o c}^{p}\left(\mathbb{R}^{N}\right)$ setting, of lower and upper semicontinuity properties of the variational eigenvalues defined in Section 6. So far, the variational eigenvalues are just inf-sup values. In Section 8 we prove that they can be also defined with respect to a suitable reflexive Banach space where the results of Section 4 apply. In particular, each inf-sup value is an eigenvalue with a corresponding eigenvector. Finally, in Section 9, we apply the theory developed in the previous sections to the case of $p$-quasi open sets and of Schrödinger potentials, thus proving the main results of the paper, Theorems 1.3 and 1.4.

\section{Notations AND PRELIMINARIES}

Throughout the paper, we fix an integer $N \geq 1$ and $1<p<\infty$. We denote by $\mathcal{L}^{N}$ the $N$-dimensional Lebesgue measure and, if $p<N$, by $p^{*}=\frac{N p}{N-p}$ the critical Sobolev exponent. We will usually write $\int$ instead of $\int_{\mathbb{R}^{N}}$. For every real number $s$, we denote by $s^{ \pm}:=\max \{ \pm s, 0\}$ its positive and negative parts. If $(X, d)$ is a metric space, we set $B_{r}(x):=\{y \in X: d(y, x)<r\}$ and we denote by $\mathcal{B}(X)$ the family of Borel subsets of $X$.

Capacity, quasi open sets and fine topology. We need to introduce the notion of $p$-capacity; we refer to [30, Chapter 2], to [29] and to [33] for more details.

Definition 2.1. For every subset $E$ of $\mathbb{R}^{N}$, the $p$-capacity of $E$ in $\mathbb{R}^{N}$ is defined as

$$
\begin{aligned}
& \operatorname{cap}_{p}(E):=\inf \left\{\int\left(|\nabla u|^{p}+|u|^{p}\right) d \mathcal{L}^{N}: u \in W^{1, p}\left(\mathbb{R}^{N}\right)\right. \\
& \left.\qquad 0 \leq u \leq 1 \mathcal{L}^{N} \text {-a.e. on } \mathbb{R}^{N}, u=1 \mathcal{L}^{N} \text {-a.e. on an open set containing } E\right\}
\end{aligned}
$$

where we agree that $\inf \emptyset=+\infty$. If $E \subseteq \mathbb{R}^{N}$, we say that a property $\mathcal{P}(x)$ holds cap -quasi everywhere in $E$, if it holds for all $x \in E$ except at most a set of zero $p$-capacity. We will write q.e. in $E$ instead of cap $_{p}$-quasi everywhere in $E$, for the sake of simplicity. 
Definition 2.2. A subset $A$ of $\mathbb{R}^{N}$ is said to be $p$-quasi open if, for every $\varepsilon>0$, there exists an open subset $\omega_{\varepsilon}$ of $\mathbb{R}^{N}$ such that $\operatorname{cap}_{p}\left(\omega_{\varepsilon}\right)<\varepsilon$ and $A \cup \omega_{\varepsilon}$ is open in $\mathbb{R}^{N}$.

Remark 2.3. First of all, we note that the open sets $\omega_{\varepsilon}$ in the above definition can be chosen to be nondecreasing, i.e. if $\varepsilon_{1} \leq \varepsilon_{2}$, then $\omega_{\varepsilon_{1}} \subseteq \omega_{\varepsilon_{2}}$. Then, for every $p$-quasi open subset $A$ of $\mathbb{R}^{N}$, it is possible to check from the definition that there exist two Borel and $p$-quasi open sets $G_{1}, G_{2}$ and two sets of zero p-capacity $E_{1}, E_{2}$ such that $A=G_{1} \cup E_{1}=G_{2} \backslash E_{2}$. For example, with $\omega_{\varepsilon}$ as in Definition 2.2, one can take $G_{1}=A \backslash\left(\bigcap_{j \in \mathbb{N}} \omega_{1 / j}\right)=\bigcup_{j \in \mathbb{N}}\left(\left(A \cup \omega_{1 / j}\right) \backslash \omega_{1 / j}\right), E_{1}=A \cap\left(\bigcap_{j \in \mathbb{N}} \omega_{1 / j}\right), G_{2}=\cap_{j \in \mathbb{N}}\left(A \cup \omega_{1 / j}\right)$ and $E_{2}=G_{2} \backslash A$.

Definition 2.4. A function $u: \mathbb{R}^{N} \rightarrow \overline{\mathbb{R}}$ is said to be $p$-quasi continuous ( -quasi lower semicontinuous, p-quasi upper semicontinuous, resp.) if for every $\varepsilon>0$ there exists an open subset $\omega_{\varepsilon}$ of $\mathbb{R}^{N}$ with $\operatorname{cap}_{p}\left(\omega_{\varepsilon}\right)<\varepsilon$ such that $\left.u\right|_{\mathbb{R}^{N} \backslash \omega_{\varepsilon}}$ is continuous (lower semicontinuous, upper semicontinuous, resp.).

Remark 2.5. It can be proved (see [5, Proposition IV.2 (d)] for the case $p=2$ ) that a function $u$ : $\mathbb{R}^{N} \rightarrow \overline{\mathbb{R}}$ is $p$-quasi lower (resp. $p$-quasi upper) semicontinuous if and only if the sets $\left\{x \in \mathbb{R}^{N}: u(x)>t\right\}$ (resp. $\left\{x \in \mathbb{R}^{N}: u(x)<t\right\}$ ) are $p$-quasi open for every $t \in \mathbb{R}$.

For every $u \in W_{l o c}^{1, p}\left(\mathbb{R}^{N}\right)$, there exists a Borel and $p$-quasi continuous representative $\tilde{u}: \mathbb{R}^{N} \rightarrow \mathbb{R}$ of $u$ and, if $\tilde{u}$ and $\hat{u}$ are two $p$-quasi continuous representatives of the same $u$, then we have $\tilde{u}=\hat{u}$ q.e. in $\mathbb{R}^{N}$. In the following, for every $u \in W_{l o c}^{1, p}\left(\mathbb{R}^{N}\right)$, we will consider only its Borel and $p$-quasi continuous representatives.

Definition 2.6. If $A$ is a $p$-quasi open subset of $\mathbb{R}^{N}$, we set

$$
W_{0}^{1, p}(A):=\left\{u \in W^{1, p}\left(\mathbb{R}^{N}\right): u=0 \text { q.e. in } \mathbb{R}^{N} \backslash A\right\} .
$$

It turns out that the above definition is naturally equivalent to the usual one, if $A$ is an open subset of $\mathbb{R}^{N}$. In the following, we also denote by $W_{c}^{1, p}\left(\mathbb{R}^{N}\right)$ the set of $u$ 's in $W^{1, p}\left(\mathbb{R}^{N}\right)$ vanishing q.e. outside some compact subset of $\mathbb{R}^{N}$.

From now on in this paragraph, we restrict ourselves to the case $p \leq N$, since if $p>N$ every point $x \in \mathbb{R}^{N}$ has positive $p$-capacity, thus $p$-quasi open sets coincide with Euclidean open sets.

Although $p$-quasi open subsets do not form a topology on $\mathbb{R}^{N}$ (because an uncountable union of $p$-quasi open subsets is not always $p$-quasi open), it is possible to define the $p$-fine topology, which turns out to be a useful tool from nonlinear potential theory. In the present work we recall only the basic notions and properties that we need, and refer to $[29,30]$ and the references therein for more details.

Definition 2.7. A subset $W$ of $\mathbb{R}^{N}$ is said to be $p$-finely open if for every $x \in W$ we have

$$
\int_{0}^{1}\left(\frac{\operatorname{cap}_{p}\left(B_{r}(x) \backslash W\right)}{r^{N-p}}\right)^{\frac{1}{p-1}} \frac{d r}{r}<+\infty .
$$

The $p$-finely open subsets form a topology called the $p$-fine topology, which can be equivalently defined as the coarsest topology making all $p$-superharmonic functions continuous.

We recall now the properties of the $p$-fine topology we need. In particular, we refer to [29, Theorem 2.3] for the quasi-Lindelöf property.

Proposition 2.8. The p-fine topology has the quasi-Lindelöf property, that is: for each family $\mathcal{W}$ of p-finely open sets, there is a countable subfamily $\mathcal{W}^{\prime}$ such that

$$
\operatorname{cap}_{p}\left(\bigcup_{W \in \mathcal{W}} W \backslash \bigcup_{W \in \mathcal{W}^{\prime}} W\right)=0 .
$$

Moreover, for every subset $A$ of $\mathbb{R}^{N}$, the following are equivalent:

(a) $A$ is Borel and p-quasi open;

(b) $A=W \cup E$, with $W, E$ Borel, $W$ p-finely open and $\operatorname{cap}_{p}(E)=0$;

(c) there exists $u \in W_{\text {loc }}^{1, p}\left(\mathbb{R}^{N}\right)$ such that $A=\{u>0\}$.

Remark 2.9. From Remark 2.3 and Proposition 2.8 we infer that, for every $p$-quasi open subset $A$ of $\mathbb{R}^{N}$, there exist a Borel and $p$-finely open set $W$ and a set of zero $p$-capacity $E$ such that $A=W \cup E$. 
Basic definitions about $\Gamma$-convergence. Before stating the definition of $\Gamma$-convergence, we recall that, given a topological space $\mathcal{X}$ and a function $f: \mathcal{X} \rightarrow \overline{\mathbb{R}}$, the lower semicontinuous envelope of $f$ is defined as

$$
\mathrm{sc}^{-} f:=\sup \{g: g: \mathcal{X} \rightarrow \overline{\mathbb{R}} \text { is lower semicontinuous and } g \leq f\} .
$$

We start with the topological definition of $\Gamma$-convergence (see [18]).

Definition 2.10. Let $\mathcal{X}$ be a topological space and $\mathcal{N}(u)$ the family of all open neighborhoods of a point $u \in \mathcal{X}$. Given a sequence of functions $f_{n}: \mathcal{X} \rightarrow \overline{\mathbb{R}}$ with $n \in \mathbb{N}$, we define

$$
\begin{array}{lll}
\left(\Gamma-\liminf _{n \rightarrow \infty} f_{n}\right)(u):=\sup _{U \in \mathcal{N}(u)} \liminf _{n \rightarrow \infty} \inf _{v \in U} f_{n}(v) & \text { for all } u \in \mathcal{X}, \\
\left(\Gamma-\limsup _{n \rightarrow \infty} f_{n}\right)(u):=\sup _{U \in \mathcal{N}(u)} \limsup _{n \rightarrow \infty} \inf _{v \in U} f_{n}(v) & \text { for all } u \in \mathcal{X} .
\end{array}
$$

At each $u \in \mathcal{X}$ satisfying

$$
\left(\Gamma-\liminf _{n \rightarrow \infty} f_{n}\right)(u)=\left(\Gamma-\limsup _{n \rightarrow \infty} f_{n}\right)(u),
$$

we denote by $\left(\Gamma-\lim _{n \rightarrow \infty} f_{n}\right)(u)$ the common value of $\left(\Gamma-\liminf _{n \rightarrow \infty} f_{n}\right)(u)$ and $\left(\Gamma-\limsup _{n \rightarrow \infty} f_{n}\right)(u)$.

Given $f: \mathcal{X} \rightarrow \overline{\mathbb{R}}$, we say that $\left(f_{n}\right)$ is $\Gamma$-convergent to $f$ in $\mathcal{X}$, if

$$
f(u)=\left(\Gamma-\lim _{n \rightarrow \infty} f_{n}\right)(u) \quad \text { for all } u \in \mathcal{X} .
$$

If $\mathcal{X}$ is metrizable, then the following properties hold:

- for every $u_{n} \rightarrow u$, we have

$$
\left(\Gamma-\liminf _{n \rightarrow \infty} f_{n}\right)(u) \leq \liminf _{n \rightarrow \infty} f_{n}\left(u_{n}\right) ;
$$

- for every $u \in \mathcal{X}$ there exists a recovery sequence $u_{n} \rightarrow u$ such that

$$
\left(\Gamma-\liminf _{n \rightarrow \infty} f_{n}\right)(u)=\liminf _{n \rightarrow \infty} f_{n}\left(u_{n}\right)
$$

- for every $u_{n} \rightarrow u$, we have

$$
\left(\Gamma-\limsup _{n \rightarrow \infty} f_{n}\right)(u) \leq \limsup _{n \rightarrow \infty} f_{n}\left(u_{n}\right)
$$

- for every $u \in \mathcal{X}$ there exists a recovery sequence $u_{n} \rightarrow u$ such that

$$
\left(\Gamma-\limsup _{n \rightarrow \infty} f_{n}\right)(u)=\limsup _{n \rightarrow \infty} f_{n}\left(u_{n}\right) .
$$

\section{Convergence OF FUnCtionals AND OF INF-SUP VAlueS}

In this section we develop some results of [22]. We consider an index $i$ with the following properties:

(a) $\quad i(K)$ is an integer greater or equal than 1 and is defined whenever $K$ is a nonempty, compact and symmetric subset of a metrizable topological vector space $\mathcal{X}$ such that $0 \notin K$;

(b) if $K \subseteq \mathcal{X} \backslash\{0\}$ is nonempty, compact and symmetric, then there exists an open subset $U$ of $\mathcal{X} \backslash\{0\}$ such that $K \subseteq U$ and

$$
i(\widehat{K}) \leq i(K) \quad \text { for all nonempty, compact and symmetric } \widehat{K} \subseteq U ;
$$

(c) if $K_{1}, K_{2} \subseteq \mathcal{X} \backslash\{0\}$ are nonempty, compact and symmetric, then

$$
i\left(K_{1} \cup K_{2}\right) \leq i\left(K_{1}\right)+i\left(K_{2}\right) ;
$$

(d) if $\mathcal{Y}$ also is a metrizable topological vector space, $K \subseteq \mathcal{X} \backslash\{0\}$ is nonempty, compact and symmetric and $\pi: K \rightarrow \mathcal{Y} \backslash\{0\}$ is continuous and odd, then we have $i(\pi(K)) \geq i(K)$;

(e) if $\mathcal{X}$ is a real normed space with $1 \leq \operatorname{dim} \mathcal{X}<\infty$, then we have

$$
i(\{u \in \mathcal{X}:\|u\|=1\})=\operatorname{dim} \mathcal{X} .
$$


Well known examples are the Krasnosel'skii genus (see e.g. [34, 37]) and the $\mathbb{Z}_{2}$-cohomological index (see $[25,26])$. More general examples are contained in [4].

Throughout this section, $\mathcal{X}$ will denote a metrizable and locally convex topological vector space. We also denote by $\mathcal{K}$ the family of nonempty and compact subsets of $\mathcal{X} \backslash\{0\}$ endowed with the metrizable topology of the Hausdorff convergence (see e.g. [2, Definition 4.4.9]). Finally, for every integer $m \geq 1$, we denote by $\mathcal{K}_{m}$ the family of nonempty, compact and symmetric subsets $K$ of $\mathcal{X} \backslash\{0\}$ such that $i(K) \geq m$.

Assume we also have the even functionals

$$
R^{(n)}, R: \mathcal{X} \backslash\{0\} \rightarrow[0,+\infty], \quad \text { where } n \in \mathbb{N},
$$

and define $\mathcal{R}_{m}^{(n)}: \mathcal{K} \rightarrow[0,+\infty]$ by

$$
\mathcal{R}_{m}^{(n)}(K)= \begin{cases}\sup _{u \in K} R^{(n)}(u) & \text { if } K \in \mathcal{K}_{m} \\ +\infty & \text { otherwise }\end{cases}
$$

and $\mathcal{R}_{m}: \mathcal{K} \rightarrow[0,+\infty]$ in the analogous way with $R$ instead of $R^{(n)}$.

The next result is a simple adaptation of [22, Theorem 4.2 and Corollary 4.3]. We provide the proof for reader's convenience. Let us point out that [22] extended by a general abstract approach previous results of [14]. However, the main result of this last paper, namely [14, Theorem 3.3], requires an upper estimate (assumption (A2)) which is not compatible with the case of moving (quasi-)open subsets of a given open set $\Omega$.

Theorem 3.1. If we have

$$
R(u) \leq\left(\Gamma-\liminf _{n \rightarrow \infty} R^{(n)}\right)(u) \quad \text { for all } u \in \mathcal{X} \backslash\{0\}
$$

then it is

$$
\mathcal{R}_{m}(K) \leq\left(\Gamma-\liminf _{n \rightarrow \infty} \mathcal{R}_{m}^{(n)}\right)(K) \quad \text { for all } m \geq 1 \text { and } K \in \mathcal{K}
$$

If we also have that:

- for every strictly increasing sequence $\left(n_{k}\right)$ in $\mathbb{N}$ and every sequence $\left(u^{(k)}\right)$ in $\mathcal{X} \backslash\{0\}$ with

$$
\sup _{k} R^{\left(n_{k}\right)}\left(u^{(k)}\right)<+\infty,
$$

there exists a subsequence $\left(u^{\left(k_{j}\right)}\right)$ converging to some $u \neq 0$,

then it is also

$$
\begin{aligned}
& \inf _{K \in \mathcal{K}} \mathcal{R}_{m}(K) \quad \leq \liminf _{n \rightarrow \infty}\left(\inf _{K \in \mathcal{K}} \mathcal{R}_{m}^{(n)}(K)\right) \\
& \inf _{K \in \mathcal{K}_{m}} \sup _{u \in K} R(u) \leq \liminf _{n \rightarrow \infty}\left(\inf _{K \in \mathcal{K}_{m}} \sup _{u \in K} R^{(n)}(u)\right)
\end{aligned}
$$

for all $m \geq 1$, where we agree that $\inf \emptyset=+\infty$.

Proof. Let $m \geq 1$, let $K \in \mathcal{K}$ and let $\left(K^{(n)}\right)$ be a sequence Hausdorff converging to $K$ such that

$$
\liminf _{n \rightarrow \infty} \mathcal{R}_{m}^{(n)}\left(K^{(n)}\right)=\left(\Gamma-\liminf _{n \rightarrow \infty} \mathcal{R}_{m}^{(n)}\right)(K) .
$$

Without loss of generality, we may assume that this value is not $+\infty$. Let $\lambda \in \mathbb{R}$ with

$$
\lambda>\liminf _{n \rightarrow \infty} \mathcal{R}_{m}^{(n)}\left(K^{(n)}\right) .
$$

Then there exists a subsequence $\left(K^{\left(n_{k}\right)}\right)$ such that

$$
\sup _{k \in \mathbb{N}} \sup _{u \in K^{\left(n_{k}\right)}} R^{\left(n_{k}\right)}(u)=\sup _{k \in \mathbb{N}} \mathcal{R}_{m}^{\left(n_{k}\right)}\left(K^{\left(n_{k}\right)}\right)<\lambda .
$$

In particular, $K^{\left(n_{k}\right)} \in \mathcal{K}_{m}^{\left(n_{k}\right)}$ so that $K$ also is symmetric.

On the other hand, for every $u \in K$, there exists $u^{(n)} \in K^{(n)}$ with $u^{(n)} \rightarrow u$, whence

$$
R(u) \leq \liminf _{n \rightarrow \infty} R^{(n)}\left(u^{(n)}\right) \leq \liminf _{k \rightarrow \infty} R^{\left(n_{k}\right)}\left(u^{\left(n_{k}\right)}\right) \leq \lambda,
$$


which implies that

$$
\sup _{u \in K} R(u) \leq \lambda
$$

Let $U$ be an open subset of $\mathcal{X} \backslash\{0\}$ such that $K \subseteq U$ and

$$
i(\widehat{K}) \leq i(K)
$$

for all nonempty, compact and symmetric subset $\widehat{K}$ of $U$. Since $K^{\left(n_{k}\right)} \subseteq U$ eventually as $k \rightarrow \infty$, we have $i\left(K^{\left(n_{k}\right)}\right) \leq i(K)$ eventually as $k \rightarrow \infty$, whence $i(K) \geq m$. Therefore, it is $K \in \mathcal{K}_{m}$ and

$$
\mathcal{R}_{m}(K)=\sup _{u \in K} R(u) \leq \lambda \text {. }
$$

By the arbitrariness of $\lambda$, it follows that

$$
\mathcal{R}_{m}(K) \leq\left(\Gamma-\liminf _{n \rightarrow \infty} \mathcal{R}_{m}^{(n)}\right)(K) .
$$

Assume now that, for every strictly increasing sequence $\left(n_{k}\right)$ in $\mathbb{N}$ and every sequence $\left(u^{(k)}\right)$ in $\mathcal{X} \backslash\{0\}$ with

$$
\sup _{k} R^{\left(n_{k}\right)}\left(u^{(k)}\right)<+\infty
$$

there exists a subsequence $\left(u^{\left(k_{j}\right)}\right)$ converging to some $u \neq 0$.

Then the sequence $\left(R^{(n)}\right)$ is asymptotically equicoercive in the sense of [22, Definition 2.3]. From [22, Proposition 2.5] we infer that the sequence $\left(\mathcal{R}_{m}^{(n)}\right)$ is asymptotically equicoercive with respect to the Hausdorff convergence. From [22, Proposition 2.4] we conclude that

$$
\inf _{K \in \mathcal{K}} \mathcal{R}_{m}(K) \leq \liminf _{n \rightarrow \infty}\left(\inf _{K \in \mathcal{K}} \mathcal{R}_{m}^{(n)}(K)\right)
$$

namely

$$
\inf _{K \in \mathcal{K}_{m}} \sup _{u \in K} R(u) \leq \liminf _{n \rightarrow \infty}\left(\inf _{K \in \mathcal{K}_{m}} \sup _{u \in K} R^{(n)}(u)\right)
$$

and the proof is complete.

Now we consider the particular case in which

$$
R^{(n)}(u)= \begin{cases}f^{(n)}(u) & \text { if } 1+g_{2}^{(n)}(u) \leq g_{1}^{(n)}(u)<+\infty \\ +\infty & \text { otherwise }\end{cases}
$$

where

$$
f^{(n)}, g_{1}^{(n)}, g_{2}^{(n)}: \mathcal{X} \rightarrow[0,+\infty]
$$

are even functionals, and $R$ is defined in the analogous way with respect to the even functionals

$$
f, g_{1}, g_{2}: \mathcal{X} \rightarrow[0,+\infty]
$$

For every $E \subseteq \mathcal{X}$, define also $I_{E}: \mathcal{X} \rightarrow[0,+\infty]$ by

$$
I_{E}(u)= \begin{cases}0 & \text { if } u \in E \\ +\infty & \text { otherwise }\end{cases}
$$

Corollary 3.2. Assume that:

(a) the functionals $f^{(n)}, f, g_{1}^{(n)}, g_{1}$ and $g_{2}^{(n)}, g_{2}$ are positively homogeneous of the same degree $\alpha>0$;

(b) we have

$$
\begin{array}{r}
\left(f+\lambda g_{2}+I_{\left\{g_{1}<+\infty\right\}}\right)(u) \leq\left(\Gamma-\liminf _{n \rightarrow \infty}\left(f^{(n)}+\lambda g_{2}^{(n)}+I_{\left\{g_{1}^{(n)}<+\infty\right\}}\right)\right)(u) \\
\text { for all } \lambda>0 \text { and } u \in \mathcal{X} \backslash\{0\} ;
\end{array}
$$


(c) for every strictly increasing sequence $\left(n_{k}\right)$ in $\mathbb{N}$ and every sequence $\left(u^{(k)}\right)$ in $\mathcal{X} \backslash\{0\}$ with $\sup _{k} f^{\left(n_{k}\right)}\left(u^{(k)}\right)<+\infty, \quad \sup _{k} g_{1}^{\left(n_{k}\right)}\left(u^{(k)}\right)<+\infty, \quad g_{2}^{\left(n_{k}\right)}\left(u^{(k)}\right)<g_{1}^{\left(n_{k}\right)}\left(u^{(k)}\right) \quad$ for all $k \in \mathbb{N}$,

there exists a subsequence $\left(u^{\left(k_{j}\right)}\right)$ converging in $\mathcal{X}$ to some $u$ satisfying

$$
g_{1}(u) \geq \limsup _{j \rightarrow \infty} g_{1}^{\left(n_{k_{j}}\right)}\left(u^{\left(k_{j}\right)}\right) ;
$$

(d) we have $g_{1}(0)=0$ and $f(u)>0$ for all $u \neq 0$ with $g_{2}(u) \leq g_{1}(u)<+\infty$.

Then, for every $m \geq 1$, we have

$$
\begin{aligned}
& \inf _{K \in \mathcal{K}} \mathcal{R}_{m}(K) \quad \leq \liminf _{n \rightarrow \infty}\left(\inf _{K \in \mathcal{K}} \mathcal{R}_{m}^{(n)}(K)\right) \\
& \inf _{K \in \mathcal{K}_{m}} \sup _{u \in K} R(u) \leq \liminf _{n \rightarrow \infty}\left(\inf _{K \in \mathcal{K}_{m}} \sup _{u \in K} R^{(n)}(u)\right)
\end{aligned}
$$

Proof. We aim to apply Theorem 3.1.

I) First of all we claim that, if $\left(u^{(n)}\right)$ is a sequence converging to $u$ in $\mathcal{X} \backslash\{0\}$ with

$$
\sup _{n} f^{(n)}\left(u^{(n)}\right)<+\infty, \sup _{n} g_{2}^{(n)}\left(u^{(n)}\right)<+\infty, g_{1}^{(n)}\left(u^{(n)}\right)<+\infty \text { for all } n \in \mathbb{N},
$$

then we have

$$
f(u) \leq \liminf _{n \rightarrow \infty} f^{(n)}\left(u^{(n)}\right), \quad g_{2}(u) \leq \liminf _{n \rightarrow \infty} g_{2}^{(n)}\left(u^{(n)}\right), \quad g_{1}(u)<+\infty .
$$

Actually, by assumption $(b)$ we have $g_{1}(u)<+\infty$ and, for every $\lambda>0$,

$$
\begin{aligned}
f(u) \leq f(u)+\lambda g_{2}(u) \quad \leq \liminf _{n \rightarrow \infty}\left(f^{(n)}\left(u^{(n)}\right)+\lambda g_{2}^{(n)}\left(u^{(n)}\right)\right) \\
\leq \liminf _{n \rightarrow \infty} f^{(n)}\left(u^{(n)}\right)+\lambda \limsup _{n \rightarrow \infty} g_{2}^{(n)}\left(u^{(n)}\right), \\
g_{2}(u) \leq \frac{1}{\lambda}\left(f(u)+\lambda g_{2}(u) \leq \frac{1}{\lambda} \liminf _{n \rightarrow \infty}\left(f^{(n)}\left(u^{(n)}\right)+\lambda g_{2}^{(n)}\left(u^{(n)}\right)\right)\right. \\
\leq \frac{1}{\lambda} \limsup _{n \rightarrow \infty} f^{(n)}\left(u^{(n)}\right)+\liminf _{n \rightarrow \infty} g_{2}^{(n)}\left(u^{(n)}\right) .
\end{aligned}
$$

By the arbitrariness of $\lambda$ the claim follows.

II) Assume now that $\left(n_{k}\right)$ is a strictly increasing sequence in $\mathbb{N}$ and $\left(u^{(k)}\right)$ a sequence in $\mathcal{X} \backslash\{0\}$ such that

$$
\sup _{k} R^{\left(n_{k}\right)}\left(u^{(k)}\right)<+\infty \text {. }
$$

We aim to show that there exists a subsequence $\left(u^{\left(k_{j}\right)}\right)$ converging to some $u$ in $\mathcal{X} \backslash\{0\}$.

Actually, we have $1+g_{2}^{\left(n_{k}\right)}\left(u^{(k)}\right) \leq g_{1}^{\left(n_{k}\right)}\left(u^{(k)}\right)<+\infty$ and

$$
\sup _{k} f^{\left(n_{k}\right)}\left(u^{(k)}\right)<+\infty \text {. }
$$

First we show that $\left(g_{1}^{\left(n_{k}\right)}\left(u^{(k)}\right)\right)$ is bounded. Assume for the sake of contradiction that, up to a subsequence,

$$
\lim _{k \rightarrow \infty} g_{1}^{\left(n_{k}\right)}\left(u^{(k)}\right)=+\infty
$$

so that a suitably rescaled sequence $\left(v^{(k)}\right)$ satisfies

$$
\lim _{k \rightarrow \infty} f^{\left(n_{k}\right)}\left(v^{(k)}\right)=0, \quad g_{2}^{\left(n_{k}\right)}\left(v^{(k)}\right)<g_{1}^{\left(n_{k}\right)}\left(v^{(k)}\right)=1 \quad \text { for all } k \in \mathbb{N} .
$$

By assumption $(c)$, up to a further subsequence $\left(v^{(k)}\right)$ is convergent in $\mathcal{X}$ to some $v$ satisfying $g_{1}(v) \geq 1$, whence $v \neq 0$ by assumption $(d)$. Then by step I we have

$$
f(v)=0, \quad g_{2}(v) \leq 1 \leq g_{1}(v)<+\infty
$$

and a contradiction follows again by assumption $(d)$. Therefore $\left(g_{1}^{\left(n_{k}\right)}\left(u^{(k)}\right)\right)$ is bounded. 
Again by assumption $(c)$ we infer that there exists a subsequence $\left(u^{\left(k_{j}\right)}\right)$ converging in $\mathcal{X}$ to some $u$ satisfying

$$
1 \leq \limsup _{j \rightarrow \infty} g_{1}^{\left(n_{k_{j}}\right)}\left(u^{\left(k_{j}\right)}\right) \leq g_{1}(u)
$$

whence $u \neq 0$.

III) Finally, let $u$ in $\mathcal{X} \backslash\{0\}$ and let $\left(u^{(n)}\right)$ be a sequence converging to $u$ such that

$$
\liminf _{n \rightarrow \infty} R^{(n)}\left(u^{(n)}\right)=\left(\Gamma-\liminf _{n \rightarrow \infty} R^{(n)}\right)(u) .
$$

If

$$
\liminf _{n \rightarrow \infty} R^{(n)}\left(u^{(n)}\right)<b<+\infty,
$$

namely $1+g_{2}^{(n)}\left(u^{(n)}\right) \leq g_{1}^{(n)}\left(u^{(n)}\right)<+\infty$ and

$$
\liminf _{n \rightarrow \infty} f^{(n)}\left(u^{(n)}\right)<b,
$$

up to a subsequence, we have

$$
\sup _{n} f^{(n)}\left(u^{(n)}\right)<b \text {. }
$$

Then, as in step II, we infer that $\left(g_{1}^{(n)}\left(u^{(n)}\right)\right)$ is bounded. From step I and assumption $(c)$ it follows that

$$
f(u) \leq b, \quad g_{2}(u) \leq \liminf _{n \rightarrow \infty} g_{2}^{(n)}\left(u^{(n)}\right), \quad \limsup _{n \rightarrow \infty} g_{1}^{(n)}\left(u^{(n)}\right) \leq g_{1}(u)<+\infty .
$$

Therefore $1+g_{2}(u) \leq g_{1}(u)<+\infty$ and

$$
R(u)=f(u) \leq b
$$

From the arbitrariness of $b$ we infer that

$$
R(u) \leq\left(\Gamma-\liminf _{n \rightarrow \infty} R^{(n)}\right)(u) \quad \text { for all } u \in \mathcal{X} \backslash\{0\} .
$$

Then the assertion follows by Theorem 3.1.

The next results are a variant of $\left[22\right.$, Theorem 4.1]. However, because of the presence of $g_{2}^{(n)}, g_{2}$, a more involved argument is required.

Let us introduce the subfamily $\mathcal{K}_{m}^{f i n}$ of $K^{\prime}$ s in $\mathcal{K}_{m}$ such that $K$ is included in some finite dimensional subspace of $\mathcal{X}$.

Lemma 3.3. There exists a compatible distance $d$ on $\mathcal{X}$ such that $d(-u,-v)=d(u, v)$ and such that $B_{r}(u)$ is convex for all $u, v \in \mathcal{X}$ and $r>0$.

Moreover, for every nonempty, compact and symmetric $K \subseteq \mathcal{X} \backslash\{0\}$ and every $r>0$, there exist a finite and symmetric subset $F$ of $K$ and a continuous map

such that

$$
\begin{aligned}
& F \times \mathcal{X} \quad \longrightarrow \quad[0,1] \\
& (v, u) \quad \mapsto \quad \vartheta_{v}(u)
\end{aligned}
$$

$$
\begin{gathered}
\vartheta_{v}(u)=0 \text { whenever } d(u, v) \geq r, \\
\sum_{v \in F} \vartheta_{v}(u)=1 \text { for all } u \in K, \\
\sum_{v \in F} \vartheta_{v}(u) \leq 1 \text { for all } u \in \mathcal{X}, \\
\vartheta_{-v}(u)=\vartheta_{v}(-u) \text { for all } v \in F \text { and } u \in \mathcal{X} .
\end{gathered}
$$

Proof. It is the first part of the proof of [22, Proposition 3.1].

Theorem 3.4. Assume that:

(a) the functionals $f^{(n)}, f, g_{1}^{(n)}, g_{1}$ and $g_{2}^{(n)}, g_{2}$ are convex and positively homogeneous of the same degree $\alpha \geq 1$; 
(b) we have

$$
\begin{array}{r}
\left(f+\lambda g_{2}+I_{\left\{g_{1}<+\infty\right\}}\right)(u) \geq\left(\Gamma-\limsup _{n \rightarrow \infty}\left(f^{(n)}+\lambda g_{2}^{(n)}+I_{\left\{g_{1}^{(n)}<+\infty\right\}}\right)\right)(u) \\
\quad \text { for all } \lambda>0 \text { and } u \in \mathcal{X} \backslash\{0\} ;
\end{array}
$$

(c) for every strictly increasing sequence $\left(n_{k}\right)$ in $\mathbb{N}$ and every sequence $\left(u^{(k)}\right)$ converging to $u$ in $\mathcal{X} \backslash\{0\}$ with

we have

$$
\sup _{k} f^{\left(n_{k}\right)}\left(u^{(k)}\right)<+\infty, \quad \sup _{k} g_{2}^{\left(n_{k}\right)}\left(u^{(k)}\right)<+\infty,
$$

$$
g_{1}(u) \leq \liminf _{k \rightarrow \infty} g_{1}^{\left(n_{k}\right)}\left(u^{(k)}\right)
$$

Then, for every $m \geq 1$, we have

$$
\inf _{K \in \mathcal{K}_{m}^{f i n}} \sup _{u \in K} R(u) \geq \limsup _{n \rightarrow \infty}\left(\inf _{K \in \mathcal{K}_{m}^{f i n}} \sup _{u \in K} R^{(n)}(u)\right) .
$$

Proof. Let $d$ be a distance as in Lemma 3.3 , let $K \in \mathcal{K}_{m}^{\text {fin }}$ and $\lambda$ with

$$
\sup _{u \in K} R(u)<\lambda<+\infty \text {, }
$$

whence

$$
f(u)<\lambda \text { and } 1+g_{2}(u) \leq g_{1}(u)<+\infty \text { for all } u \in K
$$

It follows

$$
f(u)+\lambda g_{2}(u)<\lambda g_{1}(u) \quad \text { for all } u \in K .
$$

On the other hand, if we denote by $Y$ the vector subspace spanned by $K$, we have that $f, g_{1}$ and $g_{2}$ are finite, hence continuous, if restricted to $Y$ (see e.g. [24, Corollary 2.3]). Therefore, there exists $r>0$ such that $K \cap B_{r}(0)=\emptyset$ and

$$
f(v)+\lambda g_{2}(v)<\lambda g_{1}(w) \quad \text { for all } v \in K \text { and } w \in Y \text { with } d(w, v)<3 r .
$$

Let $F$ and $\vartheta$ be as in Lemma 3.3 and define an odd and continuous map $\pi: K \rightarrow Y$ by

$$
\pi(u)=\sum_{v \in F} \vartheta_{v}(u) v .
$$

Then

$$
\pi(u) \in \operatorname{conv}\{v \in F: d(v, u)<r\}, d(\pi(u), u)<r \text { and } \pi(u) \neq 0 \text { for all } u \in K
$$

whence

$$
f(v)+\lambda g_{2}(v)<\lambda g_{1}(\pi(u)) \text { for all } u, v \in K \text { with } d(u, v)<2 r .
$$

Since $F$ is a finite set, by assumption (b) there exists, for every $n \in \mathbb{N}$, an odd map $\psi^{(n)}: F \rightarrow \mathcal{X}$ such that

$$
\begin{array}{ll}
\lim _{n \rightarrow \infty} \psi^{(n)}(v)=v & \text { for all } v \in F \\
g_{1}^{(n)}\left(\psi^{(n)}(v)\right)<+\infty \text { eventually as } n \rightarrow \infty & \text { for all } v \in F, \\
f(v)+\lambda g_{2}(v) \geq \limsup _{n \rightarrow \infty}\left(f^{(n)}\left(\psi^{(n)}(v)\right)+\lambda g_{2}^{(n)}\left(\psi^{(n)}(v)\right)\right) & \text { for all } v \in F .
\end{array}
$$

If we define an odd and continuous map $\pi^{(n)}: K \rightarrow \mathcal{X}$ by

$$
\pi^{(n)}(u)=\sum_{v \in F} \vartheta_{v}(u) \psi^{(n)}(v),
$$

we have by the convexity of $f^{(n)}, g_{1}^{(n)}$ and $g_{2}^{(n)}$

$$
g_{1}^{(n)}\left(\pi^{(n)}(u)\right)<+\infty \quad \text { for all } u \in K \text {, eventually as } n \rightarrow \infty,
$$




$$
\begin{aligned}
\lim _{n \rightarrow \infty} \pi^{(n)}\left(u^{(n)}\right)=\pi(u), \quad & \limsup _{n \rightarrow \infty} \\
& f^{(n)}\left(\pi^{(n)}\left(u^{(n)}\right)\right)<+\infty, \\
& \limsup _{n \rightarrow \infty} g_{2}^{(n)}\left(\pi^{(n)}\left(u^{(n)}\right)\right)<+\infty, \quad \text { whenever } u^{(n)} \rightarrow u \text { in } K .
\end{aligned}
$$

Therefore, by assumption $(c)$ and (3.1), there exists $\bar{n} \in \mathbb{N}$ such that

$$
\pi^{(n)}(u) \neq 0, \quad f^{(n)}\left(\psi^{(n)}(v)\right)+\lambda g_{2}^{(n)}\left(\psi^{(n)}(v)\right)<\lambda g_{1}^{(n)}\left(\pi^{(n)}(u)\right)<+\infty
$$

for all $n \geq \bar{n}, u \in K$ and $v \in F$ with $d(u, v)<r$.

By the convexity of $f^{(n)}+\lambda g_{2}^{(n)}$, we infer that

$$
f^{(n)}\left(\pi^{(n)}(u)\right)+\lambda g_{2}^{(n)}\left(\pi^{(n)}(u)\right)<\lambda g_{1}^{(n)}\left(\pi^{(n)}(u)\right)<+\infty \quad \text { for all } n \geq \bar{n} \text { and } u \in K
$$

whence

$$
g_{2}^{(n)}\left(\pi^{(n)}(u)\right)<g_{1}^{(n)}\left(\pi^{(n)}(u)\right) \quad \text { for all } n \geq \bar{n} \text { and } u \in K .
$$

If we denote by $Y^{(n)}$ the vector subspace spanned by $\psi^{(n)}(F)$, we have again that $g_{1}^{(n)}$ and $g_{2}^{(n)}$ are finite, hence continuous, if restricted to $Y^{(n)}$. If we set

$$
K^{(n)}=\left\{\frac{\pi^{(n)}(u)}{\left(g_{1}^{(n)}\left(\pi^{(n)}(u)\right)-g_{2}^{(n)}\left(\pi^{(n)}(u)\right)\right)^{1 / \alpha}}: u \in K\right\},
$$

it follows that $K^{(n)}$ is included in $Y^{(n)}$ and

$$
i\left(K^{(n)}\right) \geq i(K) \geq m
$$

whence

$$
K^{(n)} \in \mathcal{K}_{m}^{f i n}, f^{(n)}(u)<\lambda \text { and } 1+g_{2}^{(n)}(u)=g_{1}^{(n)}(u)<+\infty \text { for all } n \geq \bar{n} \text { and } u \in K^{(n)} .
$$

Then

and the assertion follows by the arbitrariness of $\lambda$.

$$
\limsup _{n \rightarrow \infty}\left(\sup _{u \in K^{(n)}} R^{(n)}(u)\right) \leq \lambda
$$

Theorem 3.5. Assume that $f, g_{1}$ and $g_{2}$ are convex and positively homogeneous of the same degree $\alpha \geq 1$. Suppose also that:

(a) for every $b, a>0$ and sequences $\left(v_{k}\right)$ converging to $v$ in

$$
\left\{u \in \mathcal{X} \backslash\{0\}: f(u) \leq b, a+g_{2}(u) \leq g_{1}(u)<+\infty\right\}
$$

and $\left(w_{k}\right)$ in

$$
\left\{u \in \mathcal{X} \backslash\{0\}: f(u) \leq b, g_{2}(u) \leq b\right\}
$$

also converging to $v$, we have

$$
\begin{aligned}
& \limsup _{k \rightarrow \infty} g_{2}\left(v_{k}\right)<+\infty, \\
& \liminf _{k \rightarrow \infty}\left(g_{1}\left(w_{k}\right)-g_{2}\left(v_{k}\right)\right) \geq a .
\end{aligned}
$$

Then, for every integer $m \geq 1$, we have

$$
\inf _{K \in \mathcal{K}_{m}} \sup _{u \in K} R(u)=\inf _{K \in \mathcal{K}_{m}^{f i n}} \sup _{u \in K} R(u) .
$$

Proof. Let $d$ be again a distance as in Lemma 3.3. Of course, we have

$$
\inf _{K \in \mathcal{K}_{m}} \sup _{u \in K} R(u) \leq \inf _{K \in \mathcal{K}_{m}^{f i n}} \sup _{u \in K} R(u) .
$$

To prove the opposite inequality, let $K \in \mathcal{K}_{m}$ and $\lambda$ with

$$
\sup _{u \in K} R(u)<\lambda<+\infty
$$

and let $\varepsilon>0$ be such that

$$
\sup _{u \in K} R(u) \leq(1-\varepsilon) \lambda,
$$


namely

$$
f(u) \leq(1-\varepsilon) \lambda \text { and } 1+g_{2}(u) \leq g_{1}(u)<+\infty \text { for all } u \in K .
$$

Taking into account assumption $(a)$, there exists firstly $b>0$ such that

$$
f(u) \leq b \text { and } g_{2}(u) \leq b \text { for all } u \in K
$$

and then $r>0$ such that $K \cap B_{r}(0)=\emptyset$ and

$$
g_{1}(w)-g_{2}(v)>1-\varepsilon \quad \text { for all } v \in K \text { and } w \in \mathcal{X} \backslash\{0\}
$$

with $f(w) \leq b, g_{2}(w) \leq b$ and $d(w, v)<2 r$.

It follows

$$
f(v)+\lambda g_{2}(v)<\lambda g_{1}(w) \quad \text { for all } v \in K \text { and } w \in \mathcal{X} \backslash\{0\}
$$

with $f(w) \leq b, g_{2}(w) \leq b$ and $d(w, v)<2 r$.

Let $F$ and $\vartheta$ be as in Lemma 3.3 and define an odd and continuous map $\pi: K \rightarrow \mathcal{X}$ by

$$
\pi(u)=\sum_{v \in F} \vartheta_{v}(u) v .
$$

Then we have again

$$
\pi(u) \in \operatorname{conv}\{v \in F: d(v, u)<r\}, d(\pi(u), u)<r \text { and } \pi(u) \neq 0 \text { for all } u \in K .
$$

In particular, by the convexity of $f, g_{1}$ and $g_{2}$ it follows first that

$$
f(v)+\lambda g_{2}(v)<\lambda g_{1}(\pi(u))<+\infty \quad \text { for all } v \in F \text { and } u \in K \text { with } d(u, v)<r
$$

and then that

$$
f(\pi(u))+\lambda g_{2}(\pi(u))<\lambda g_{1}(\pi(u))<+\infty \quad \text { for all } u \in K .
$$

As before, $g_{1}$ and $g_{2}$ are continuous when restricted to the vector subspace spanned by $F$. If we set

$$
\widehat{K}=\left\{\frac{\pi(u)}{\left(g_{1}(\pi(u))-g_{2}(\pi(u))\right)^{1 / \alpha}}: u \in K\right\},
$$

it follows that

$$
i(\widehat{K}) \geq i(K) \geq m
$$

whence

$$
\widehat{K} \in \mathcal{K}_{m}^{f i n}, f(u)<\lambda \text { and } 1+g_{2}(u)=g_{1}(u)<+\infty \text { for all } u \in \widehat{K} \text {. }
$$

Therefore

$$
\sup _{u \in \widehat{K}} R(u) \leq \lambda
$$

and the assertion follows by the arbitrariness of $\lambda$.

Remark 3.6. Suppose that $f, g_{1}$ and $g_{2}$ are convex and positively homogeneous of the same degree $\alpha \geq 1$.

Then assumption $(a)$ of Theorem 3.5 is satisfied in each of the following cases:

(b) for every $b>0$, the restriction of $g_{1}$ to

$$
\left\{u \in \mathcal{X}: f(u) \leq b, g_{1}(u) \leq b, g_{2}(u) \leq b\right\}
$$

is continuous;

(c) for every $b>0$, the restriction of $g_{1}$ to

$$
\left\{u \in \mathcal{X} \backslash\{0\}: f(u) \leq b, g_{1}(u) \leq b\right\}
$$

is lower semicontinuous and $g_{2}=0$. 
Proof. Let $\left(v_{k}\right)$ and $\left(w_{k}\right)$ be two sequences as in assumption $(a)$ of Theorem 3.5. If $(b)$ holds, we first claim that $\left(g_{1}\left(v_{k}\right)\right)$ is bounded. Otherwise, up to a subsequence, a rescaled sequence $\left(u_{k}\right)$ is convergent to 0 and satisfies $f\left(u_{k}\right) \rightarrow 0$ and $g_{2}\left(u_{k}\right)<g_{1}\left(u_{k}\right)=1$. On the other hand $f(0)=g_{1}(0)=g_{2}(0)=0$ by convexity and homogeneity, whence a contradiction. Since

$$
g_{1}\left(w_{k}\right)-g_{2}\left(v_{k}\right) \geq g_{1}\left(w_{k}\right)-g_{1}\left(v_{k}\right)+a,
$$

the assertion follows.

If $(c)$ holds, we have

$$
g_{1}\left(w_{k}\right)-g_{2}\left(v_{k}\right)=g_{1}\left(w_{k}\right)
$$

with $a \leq g_{1}(v)<+\infty$ and the assertion immediately follows.

\section{Nonlinear EigenVAlue PROBLEMS}

This section is devoted to some basic facts concerning nonlinear eigenvalues problems. Up to some adaptation, our approach is inspired by [39].

Throughout this section, $X$ will denote a reflexive Banach space and

$$
\varphi, \psi_{1}, \psi_{2}: X \rightarrow \mathbb{R}
$$

three even functionals of class $C^{1}$ which are assumed to be positively homogeneous of the same degree $\alpha>1$. We aim to study the nonlinear eigenvalue problem

$$
\varphi^{\prime}(u)=\lambda\left(\psi_{1}^{\prime}(u)-\psi_{2}^{\prime}(u)\right) .
$$

Definition 4.1. We say that $u \in X$ is an eigenvector of $(4.1)$ if $\psi_{1}(u)-\psi_{2}(u) \neq 0$ and there exists $\lambda \in \mathbb{R}$ such that $(u, \lambda)$ satisfies $(4.1)$. It is easily seen that

$$
\lambda=\frac{\varphi(u)}{\psi_{1}(u)-\psi_{2}(u)}
$$

and $\lambda$ is said to be the eigenvalue associated with $u$.

In the following of this section, we consider only the eigenvectors $u$ with $\psi_{1}(u)-\psi_{2}(u)>0$ and the associated eigenvalues $\lambda$. If we set

$$
\widehat{M}=\left\{u \in X: \psi_{1}(u)-\psi_{2}(u)=1\right\}
$$

it is easily seen that $\widehat{M}$ is a symmetric hypersurface in $X \backslash\{0\}$ of class $C^{1}$ and that $\lambda$ is an eigenvalue if and only if $\lambda$ is a critical value of $\left.\varphi\right|_{\widehat{M}}$.

For the next concepts, we refer the reader to [6, 23].

Definition 4.2. Let $D \subseteq X$. A map $F: D \rightarrow X^{\prime}$ is said to be of class $(S)_{+}$if, for every sequence $\left(u_{n}\right)$ in $D$ weakly convergent to $u$ in $X$ with

$$
\limsup _{n \rightarrow \infty}\left\langle F\left(u_{n}\right), u_{n}-u\right\rangle \leq 0
$$

we have $\left\|u_{n}-u\right\| \rightarrow 0$.

If $Y$ is a topological space, a map $F: D \rightarrow Y$ is said to be completely continuous if it is continuous and, for every bounded sequence $\left(u_{n}\right)$ in $D$, the sequence $\left(F\left(u_{n}\right)\right)$ admits a convergent subsequence in $Y$.

Throughout this section, we assume that:

(ie) for every $\lambda>0$, we have that

$$
\begin{aligned}
& \text { is of class }(S)_{+} \text {, while } \\
& \varphi^{\prime}+\lambda \psi_{2}^{\prime}: X \rightarrow X^{\prime} \\
& \psi_{1}^{\prime}: X \rightarrow X^{\prime}
\end{aligned}
$$

is completely continuous with respect to the strong topology of $X^{\prime}$;

(iie) we have $\varphi(u)>0$ for all $u \neq 0$ with $\psi_{1}(u)-\psi_{2}(u) \geq 0$.

Lemma 4.3. For every $b \in \mathbb{R}$, the set

$$
\left\{u \in X: \varphi(u) \leq b, \psi_{1}(u)-\psi_{2}(u) \geq 0\right\}
$$

is bounded and we have

$$
\inf _{u \in \widehat{M}} \varphi(u)>0
$$


Proof. Let us recall that, because of assumption $(i e)$, the functional $\varphi+\lambda \psi_{2}$ is sequentially lower semicontinuous with respect to the weak topology for all $\lambda>0$ (see also [15, Proposition 3.5]), while $\psi_{1}$ is sequentially continuous with respect to the weak topology.

Let $b \in \mathbb{R}$, let $\left(u_{n}\right)$ be a sequence in $X$ with $\varphi\left(u_{n}\right) \leq b$ and $\psi_{1}\left(u_{n}\right)-\psi_{2}\left(u_{n}\right) \geq 0$ and assume, for the sake of contradiction, that

$$
\lim _{n \rightarrow \infty}\left\|u_{n}\right\|=+\infty
$$

Then a suitably rescaled sequence $\left(v_{n}\right)$ satisfies

$$
\lim _{n \rightarrow \infty} \varphi\left(v_{n}\right)=0, \quad \psi_{2}\left(v_{n}\right) \leq \psi_{1}\left(v_{n}\right),\left\|v_{n}\right\|=1 \quad \text { for all } n \in \mathbb{N} .
$$

Up to a subsequence, we may also assume that $\left(v_{n}\right)$ is weakly convergent to some $v$. For every $\lambda>0$, it follows that

$$
\begin{aligned}
\lambda \varphi(v)+\psi_{2}(v) & =\lambda\left(\varphi(v)+\lambda^{-1} \psi_{2}(v)\right) \leq \lambda \liminf _{n \rightarrow \infty}\left(\varphi\left(v_{n}\right)+\lambda^{-1} \psi_{2}\left(v_{n}\right)\right) \\
& =\lambda \lim _{n \rightarrow \infty} \varphi\left(v_{n}\right)+\liminf _{n \rightarrow \infty} \psi_{2}\left(v_{n}\right)=\liminf _{n \rightarrow \infty} \psi_{2}\left(v_{n}\right) \\
& \leq \lim _{n \rightarrow \infty} \psi_{1}\left(v_{n}\right)=\psi_{1}(v) .
\end{aligned}
$$

From the arbitrariness of $\lambda$ we infer that $\psi_{2}(v) \leq \psi_{1}(v)$ and that $\varphi(v)=0$, whence $v=0$ by assumption (iie). On the other hand, we have

$$
\begin{aligned}
\limsup _{n \rightarrow \infty}\left\langle\varphi^{\prime}\left(v_{n}\right)+\psi_{2}^{\prime}\left(v_{n}\right), v_{n}\right\rangle & =\alpha \limsup _{n \rightarrow \infty}\left(\varphi\left(v_{n}\right)+\psi_{2}\left(v_{n}\right)\right) \\
& =\alpha \lim _{n \rightarrow \infty} \varphi\left(v_{n}\right)+\alpha \limsup _{n \rightarrow \infty} \psi_{2}\left(v_{n}\right) \\
& \leq \alpha \lim _{n \rightarrow \infty} \psi_{1}\left(v_{n}\right)=\alpha \psi_{1}(v)=0
\end{aligned}
$$

whence $\left\|v_{n}\right\| \rightarrow 0$ by assumption (ie) and a contradiction follows.

Now let $\left(u_{n}\right)$ in $\widehat{M}$ be such that

$$
\lim _{n \rightarrow \infty} \varphi\left(u_{n}\right)=\inf _{u \in \widehat{M}} \varphi(u)
$$

By the previous step $\left(u_{n}\right)$ is weakly convergent, up to a subsequence, to some $u$. If $\inf _{u \in \widehat{M}} \varphi(u)=0$, arguing as before we find

$$
\lambda \varphi(u)+\psi_{2}(u) \leq \lim _{n \rightarrow \infty} \psi_{2}\left(u_{n}\right)=\lim _{n \rightarrow \infty}\left(\psi_{1}\left(u_{n}\right)-1\right)=\psi_{1}(u)-1
$$

for all $\lambda>0$, whence a contradiction. Therefore, it is $\inf _{u \in \widehat{M}} \varphi(u)>0$.

Theorem 4.4. The functional $\left.\varphi\right|_{\widehat{M}}$ is bounded from below and satisfies $(P S)_{c}$ for all $c \in \mathbb{R}$, namely every sequence $\left(u_{n}\right)$ in $\widehat{M}$ satisfying

$$
\lim _{n \rightarrow \infty} \varphi\left(u_{n}\right)=c, \quad \lim _{n \rightarrow \infty}\left\|\left(\left.\varphi\right|_{\widehat{M}}\right)^{\prime}\left(u_{n}\right)\right\|=0
$$

admits a converging subsequence.

Proof. Of course, $\left.\varphi\right|_{\widehat{M}}$ is bounded from below by assumption $($ iie $)$. To prove $(P S)_{c}$, let us recall that

$$
\left\|\left(\left.\varphi\right|_{\widehat{M}}\right)^{\prime}(u)\right\|=\min \left\{\left\|\varphi^{\prime}(u)-\lambda\left(\psi_{1}^{\prime}(u)-\psi_{2}^{\prime}(u)\right)\right\|: \lambda \in \mathbb{R}\right\} \quad \text { for all } u \in \widehat{M} .
$$

Let $\left(u_{n}\right)$ be a sequence in $\widehat{M}$ and $\left(\lambda_{n}\right)$ a sequence in $\mathbb{R}$ such that

$$
\lim _{n \rightarrow \infty} \varphi\left(u_{n}\right)=c, \quad \lim _{n \rightarrow \infty}\left\|\varphi^{\prime}\left(u_{n}\right)-\lambda_{n}\left(\psi_{1}^{\prime}\left(u_{n}\right)-\psi_{2}^{\prime}\left(u_{n}\right)\right)\right\|=0 .
$$

By Lemma 4.3 we have $c>0$ and $\left(u_{n}\right)$ is bounded hence weakly convergent, up to a subsequence, to some $u$. If we set

it follows

$$
z_{n}=\varphi^{\prime}\left(u_{n}\right)-\lambda_{n}\left(\psi_{1}^{\prime}\left(u_{n}\right)-\psi_{2}^{\prime}\left(u_{n}\right)\right)
$$

$$
\alpha \varphi\left(u_{n}\right)=\left\langle\varphi^{\prime}\left(u_{n}\right), u_{n}\right\rangle=\lambda_{n}\left\langle\psi_{1}^{\prime}\left(u_{n}\right)-\psi_{2}^{\prime}\left(u_{n}\right), u_{n}\right\rangle+\left\langle z_{n}, u_{n}\right\rangle=\lambda_{n} \alpha+\left\langle z_{n}, u_{n}\right\rangle
$$


whence

$$
\lim _{n \rightarrow \infty} \lambda_{n}=c>0 .
$$

Up to a subsequence, $\left(\psi_{1}^{\prime}\left(u_{n}\right)\right)$ is strongly convergent in $X^{\prime}$ and there exists $\lambda>0$ such that

$$
\limsup _{n \rightarrow \infty}\left(\lambda-\lambda_{n}\right)\left\langle\psi_{2}^{\prime}\left(u_{n}\right), u_{n}-u\right\rangle \leq 0 .
$$

Then we have

$$
\begin{aligned}
\limsup _{n \rightarrow \infty}\left\langle\varphi^{\prime}\left(u_{n}\right)+\lambda \psi_{2}^{\prime}\left(u_{n}\right), u_{n}\right. & -u\rangle \\
& =\limsup _{n \rightarrow \infty}\left[\left\langle\lambda_{n} \psi_{1}^{\prime}\left(u_{n}\right)+z_{n}, u_{n}-u\right\rangle+\left(\lambda-\lambda_{n}\right)\left\langle\psi_{2}^{\prime}\left(u_{n}\right), u_{n}-u\right\rangle\right] \leq 0 .
\end{aligned}
$$

From assumption (ie) we infer that $\left\|u_{n}-u\right\| \rightarrow 0$ and $(P S)_{c}$ follows.

Now let $i$ be an index as in Section 3 and define, for every $m \geq 1$,

$$
\hat{\lambda}_{m}=\inf \left\{\max _{u \in K} \varphi(u): K \text { is a nonempty, compact and symmetric subset of } \widehat{M} \text { with } i(K) \geq m\right\} \text {, }
$$

where we agree that $\hat{\lambda}_{m}=+\infty$ if there is no $K$ with $i(K) \geq m$. It is easily seen that $\hat{\lambda}_{m} \leq \hat{\lambda}_{m+1}$.

Theorem 4.5. The following facts hold:

(a) if $\widehat{M} \neq \emptyset$, which is equivalent to

$$
\left\{u \in X: \psi_{1}(u)-\psi_{2}(u)>0\right\} \neq \emptyset,
$$

then $\inf _{u \in \widehat{M}} \varphi(u)$ is achieved and

$$
\hat{\lambda}_{1}=\min _{u \in \widehat{M}} \varphi(u)
$$

(b) if there exists an odd and continuous map

$$
\left\{\xi \in \mathbb{R}^{m}:|\xi|=1\right\} \rightarrow\left\{u \in X: \psi_{1}(u)-\psi_{2}(u)>0\right\},
$$

then $\hat{\lambda}_{m}<+\infty$;

(c) if $\hat{\lambda}_{m}<+\infty$, then $\hat{\lambda}_{m}$ is an eigenvalue;

(d) if

$$
\hat{\lambda}_{m}=\cdots=\hat{\lambda}_{m+j-1}<+\infty
$$

then

$$
i\left(\left\{u \in \widehat{M}: u \text { is an eigenvector with eigenvalue } \hat{\lambda}_{m}\right\}\right) \geq j
$$

(e) we have

$$
\lim _{m \rightarrow \infty} \hat{\lambda}_{m}=+\infty .
$$

Proof. When $\widehat{M}$ is of class $C^{2}$, the assertions are well known consequences of Theorem 4.4 (see e.g. [37]). The result in the case of manifolds of class $C^{1}$ follows from $[16,38]$.

Example 4.6. Let $\varphi, \psi_{1}, \psi_{2}: \mathbb{R}^{2} \rightarrow \mathbb{R}$ be defined by

$$
\varphi(u)=\frac{1}{2}\left(u_{1}+u_{2}\right)^{2}, \quad \psi_{1}(u)=\frac{1}{2} u_{1}^{2}, \quad \psi_{2}(u)=\frac{1}{2} u_{2}^{2} .
$$

Then the problem

$$
\left\{\begin{array}{l}
u_{1}+u_{2}=\lambda u_{1} \\
u_{1}+u_{2}=-\lambda u_{2}
\end{array}\right.
$$

has no solution with $\psi_{1}(u)-\psi_{2}(u) \neq 0$ and we have

$$
\inf \left\{\varphi(u): \psi_{1}(u)-\psi_{2}(u)=1\right\}=0, \quad \varphi(u)>0 \text { for all } u \text { with } \psi_{1}(u)-\psi_{2}(u) \neq 0 .
$$

On the other hand, assumption (iie) is not satisfied. 


\section{Convergence of measures and of functionals}

In this section we introduce the notion of local $\gamma$-convergence of measures in $\mathbb{R}^{N}$ and study its properties in relation to the $\Gamma$-convergence of suitable functionals.

5.1. Convergence of capacitary measures. In the first part of this subsection we take advantage of the results of [17], where the case $p=2$ was considered. On the other hand, taking into account Proposition 2.8 , only minor changes are required in the general case.

Definition 5.1. Let $\Omega$ be an open subset of $\mathbb{R}^{N}$. We say that a non-negative Borel measure $\mu$ in $\Omega$ is $p$-capacitary if, for every $B \in \mathcal{B}(\Omega)$ with $\operatorname{cap}_{p}(B)=0$, we have $\mu(B)=0$.

A $p$-capacitary measure $\mu$ in $\Omega$ is said to be outer regular, if

$$
\mu(B)=\inf \{\mu(A): A \in \mathcal{B}(\Omega), A \supseteq B \text { and } A \text { is } p \text {-quasi open }\} \quad \text { for all } B \in \mathcal{B}(\Omega) .
$$

Definition 5.2. Two $p$-capacitary measures $\mu_{1}, \mu_{2}$ in $\Omega$ are said to be equivalent, if

$$
\mu_{1}(A)=\mu_{2}(A) \quad \text { for all } A \in \mathcal{B}(\Omega) \text { with } A \text {-quasi open } .
$$

We denote by $\mathcal{M}_{0}^{p}(\Omega)$ the quotient of the set of all $p$-capacitary measures in $\Omega$ with respect to such an equivalence relation.

Proposition 5.3. For every p-capacitary measure $\mu$ in $\Omega$, if we set

$$
\tilde{\mu}(B)=\inf \{\mu(A): A \in \mathcal{B}(\Omega), A \supseteq B \text { and } A \text { is } p \text {-quasi open }\} \quad \text { for all } B \in \mathcal{B}(\Omega) \text {, }
$$

then $\tilde{\mu}$ is an outer regular $p$-capacitary measure in $\Omega$ equivalent to $\mu$.

Moreover, if $\mu_{1}, \mu_{2}$ are two equivalent outer regular $p$-capacitary measures in $\Omega$, then $\mu_{1}=\mu_{2}$.

Proof. In the case $p=2$, see [17, Theorems 2.6, 3.9 and 3.10 and Remark 3.4].

Definition 5.4. If $\mu, \nu \in \mathcal{M}_{0}^{p}(\Omega)$, we write $\mu \leq \nu$ if

$$
\mu(A) \leq \nu(A) \quad \text { for all } A \in \mathcal{B}(\Omega) \text { with } A \text { p-quasi open } .
$$

It is easily seen that this is an order relation in $\mathcal{M}_{0}^{p}(\Omega)$.

Example 5.5. Let us provide the two most important examples of $p$-capacitary measures. The first one is given by the measure $\infty_{E}$ corresponding to a subset $E$ of $\Omega$, defined as

$$
\infty_{E}(B):=\left\{\begin{array}{ll}
0 & \text { if } \operatorname{cap}_{p}(B \cap E)=0, \\
+\infty & \text { if } \operatorname{cap}_{p}(B \cap E)>0,
\end{array} \quad \text { for all } B \in \mathcal{B}(\Omega)\right.
$$

The other one consists in a measure absolutely continuous with respect to $\mathcal{L}^{N}$, that is, for a $\mathcal{L}^{N}$-measurable function $V: \Omega \rightarrow[0,+\infty]$, the measure $V \mathcal{L}^{N}$ defined as

$$
\left(V \mathcal{L}^{N}\right)(B)=\int_{B} V d \mathcal{L}^{N} \quad \text { for all } B \in \mathcal{B}(\Omega) .
$$

On the other hand, let us see that each $p$-capacitary measure admits a decomposition incorporating contributions of this particular form.

Definition 5.6. For every $\mu \in \mathcal{M}_{0}^{p}(\Omega)$, we denote by $A_{\mu}$ the union of all Borel and $p$-finely open subsets $W$ of $\Omega$ such that $\mu(W)<+\infty$. This is called the set of $\sigma$-finiteness of $\mu$.

Since each $p$-finely open set is $p$-quasi open, the set $A_{\mu}$ is well defined and in fact $p$-finely open.

Proposition 5.7. Let $\mu \in \mathcal{M}_{0}^{p}(\Omega)$ and let $\mu_{1}, \mu_{2}$ be two representatives of $\mu$. Then the following facts hold:

(a) we have

$$
\begin{array}{ll}
\mu(A)=+\infty & \text { for all } A \in \mathcal{B}(\Omega) \text { with } \operatorname{cap}_{p}\left(A \backslash A_{\mu}\right)>0 \text { and } A \text { p-quasi open }, \\
\mu_{1}(B)=\mu_{2}(B) & \text { for all } B \in \mathcal{B}(\Omega) \text { with } \operatorname{cap}_{p}\left(B \backslash A_{\mu}\right)=0
\end{array}
$$


(b) we have that

$$
\tilde{\mu}(B)= \begin{cases}\mu(B) & \text { if } B \in \mathcal{B}(\Omega) \text { and } \operatorname{cap}_{p}\left(B \backslash A_{\mu}\right)=0, \\ +\infty & \text { if } B \in \mathcal{B}(\Omega) \text { and } \operatorname{cap}_{p}\left(B \backslash A_{\mu}\right)>0,\end{cases}
$$

is the outer regular representative of $\mu$;

(c) there exists a Borel and p-finely open subset $W$ of $A_{\mu}$ such that $\operatorname{cap}_{p}\left(A_{\mu} \backslash W\right)=0$ and, if we set

$$
\mu^{A_{\mu}}(B)=\mu(B \cap W) \quad \text { for all } B \in \mathcal{B}(\Omega),
$$

then $\mu^{A_{\mu}}$ is a $\sigma$-finite $p$-capacitary measure in $\Omega$ independent of the choice of $W$ and of the representative of $\mu$.

Proof. In the case $p=2$, see [17, Theorem 2.6, Proposition 3.16, Remark 3.13 and Theorem 3.17].

Definition 5.8. For every $\mu \in \mathcal{M}_{0}^{p}(\Omega)$, we define a $\mathcal{L}^{N}$-measurable function $V_{\mu}: \Omega \rightarrow[0,+\infty]$ by

$$
V_{\mu}= \begin{cases}\frac{d \mu^{A} \mu}{d \mathcal{L}^{N}} & \text { on } A_{\mu}, \\ +\infty & \text { on } \Omega \backslash A_{\mu},\end{cases}
$$

and we denote by $\mu_{s}$ the singular part of $\mu^{A_{\mu}}$ with respect to $\mathcal{L}^{N}$.

Proposition 5.9. The following facts hold:

(a) for every $\mu \in \mathcal{M}_{0}^{p}(\Omega)$, we have

$$
\mu(B)=\infty_{\Omega \backslash A_{\mu}}(B)+\int_{B} V_{\mu} d \mathcal{L}^{N}+\mu_{s}(B)
$$

for all $B \in \mathcal{B}(\Omega)$ with either $\operatorname{cap}_{p}\left(B \backslash A_{\mu}\right)=0$ or $B$ p-quasi open; moreover,

$$
\infty_{\Omega \backslash A_{\mu}}+V_{\mu} \mathcal{L}^{N}+\mu_{s}
$$

is the outer regular representative of $\mu$;

(b) for every $\mu, \nu \in \mathcal{M}_{0}^{p}(\Omega)$ with $\mu \leq \nu$, we have

$$
A_{\mu} \supseteq A_{\nu}, \quad V_{\mu} \leq V_{\nu} \quad \mathcal{L}^{N} \text {-a.e. in } \Omega ;
$$

(c) if $A$ is a p-quasi open subset of $\Omega$ and $\mu=\infty_{\Omega \backslash A}$, then

$$
\operatorname{cap}_{p}\left[\left(A \backslash A_{\mu}\right) \cup\left(A_{\mu} \backslash A\right)\right]=0,
$$

whence

$$
\infty_{\Omega \backslash A_{\mu}}(B)=\infty_{\Omega \backslash A}(B) \quad \text { for all } B \in \mathcal{B}(\Omega) ;
$$

(d) if $V: \Omega \rightarrow[0,+\infty]$ is $\mathcal{L}^{N}$-measurable and $\mu=V \mathcal{L}^{N}$, then

$$
\begin{array}{ll}
V_{\mu} \geq V & \mathcal{L}^{N} \text {-a.e. in } \Omega, \\
V_{\mu}=V & \mathcal{L}^{N} \text {-a.e. in } A_{\mu} ;
\end{array}
$$

(e) if $V: \Omega \rightarrow[0,+\infty]$ is p-quasi upper semicontinuous and $\mu=V \mathcal{L}^{N}$, then

$$
V_{\mu}=V \quad \mathcal{L}^{N} \text {-a.e. in } \Omega .
$$

Proof.

(a) If $\operatorname{cap}_{p}\left(B \backslash A_{\mu}\right)=0$, it follows from Proposition 5.7 and the Radon-Nikodym Theorem that

$$
\mu(B)=\infty_{\Omega \backslash A_{\mu}}(B)+\int_{B} V_{\mu} d \mathcal{L}^{N}+\mu_{s}(B),
$$

while, if $\operatorname{cap}_{p}\left(B \backslash A_{\mu}\right)>0$ and $B$ is $p$-quasi open, we have $\infty_{\Omega \backslash A_{\mu}}(B)=\mu(B)=+\infty$ by assertion $(a)$ of Proposition 5.7. In particular,

$$
\infty_{\Omega \backslash A_{\mu}}+V_{\mu} \mathcal{L}^{N}+\mu_{s}
$$

is the outer regular representative of $\mu$ by (b) of Proposition 5.7.

(b) The fact is obvious.

(c) Since $\mu^{A_{\mu}}$ is $\sigma$-finite, we have $\mu^{A_{\mu}}=0$. Then the assertion follows from $(a)$, as $A$ and $A_{\mu}$ can be supposed to be also Borel, up to a set of null $p$-capacity. 
(d) By the Radon-Nikodym Theorem, we have

$$
V_{\mu}=V \quad \mathcal{L}^{N} \text {-a.e. in } A_{\mu}
$$

while it is obvious that

(e) For every $n \in \mathbb{N}$, the set

$$
V_{\mu} \geq V \quad \mathcal{L}^{N} \text {-a.e. in } \Omega \backslash A_{\mu} .
$$

$$
\{x \in \Omega: V(x)<n\}
$$

is $p$-quasi open (see Remark 2.5). Therefore, by Remark 2.9, there exist a Borel and $p$-finely open set $W_{n}$ and $E_{n}$ with $\operatorname{cap}_{p}\left(E_{n}\right)=0$ such that

$$
\{x \in \Omega: V(x)<n\}=W_{n} \cup E_{n} .
$$

Then we have

$$
W_{n} \cap B_{n}(0) \subseteq A_{\mu}
$$

whence

$$
\{x \in \Omega: V(x)<+\infty\} \subseteq A_{\mu} \cup\left(\bigcup_{n \in \mathbb{N}} E_{n}\right)
$$

and the assertion follows from $(d)$.

Example 5.10. Let $N=1$ and $C$ be a closed subset of $\mathbb{R}$ with empty interior and $\mathcal{L}^{1}(C)>0$. If we set

$$
V= \begin{cases}0 & \text { on } C, \\ +\infty & \text { on } \Omega \backslash C,\end{cases}
$$

and consider $\mu=V \mathcal{L}^{1}$, then we have $A_{\mu}=\emptyset$, whence $V_{\mu}=+\infty \mathcal{L}^{1}$-a.e. in $\mathbb{R}$.

Remark 5.11. If $\mu \in \mathcal{M}_{0}^{p}(\Omega)$ and $u \in W_{l o c}^{1, p}(\Omega)$, then the integral

$$
\int_{\Omega}|u|^{p} d \mu
$$

is well defined, as

$$
\int_{\Omega}|u|^{p} d \mu=\int_{0}^{+\infty} \mu\left(\left\{|u|>y^{1 / p}\right\}\right) d \mathcal{L}^{1}(y)
$$

and the sets $\left\{|u|>y^{1 / p}\right\}$ are Borel and $p$-quasi open.

Then the space

$$
W_{l o c}^{1, p}(\Omega) \cap L^{p}(\Omega, \mu):=\left\{u \in W_{l o c}^{1, p}(\Omega): \int_{\Omega}|u|^{p} d \mu<+\infty\right\}
$$

is well defined and, for every $u \in W_{l o c}^{1, p}(\Omega) \cap L^{p}(\Omega, \mu)$, we have

$$
\operatorname{cap}_{p}\left(\{|u|>0\} \backslash A_{\mu}\right)=0
$$

by $(a)$ of Proposition 5.7.

Again from (a) of Proposition 5.7 we infer that the integral

$$
\int_{\Omega}|u|^{p-2} u v d \mu
$$

is well defined for all $u, v \in W_{l o c}^{1, p}(\Omega) \cap L^{p}(\Omega, \mu)$.

Moreover, if $\left(u_{n}\right)$ is a sequence in $W_{l o c}^{1, p}(\Omega) \cap L^{p}(\Omega, \mu)$ and $u \in W_{l o c}^{1, p}(\Omega) \cap L^{p}(\Omega, \mu)$, then the assertion the sequence $\left(u_{n}\right)$ is weakly convergent to $u$ in $L^{p}(\Omega, \mu)$ is independent of the choice of the representative of $\mu$.

Assume now that $\Omega$ is a bounded and open subset of $\mathbb{R}^{N}$. Here we take advantage of the results of [20]. For every $\mu \in \mathcal{M}_{0}^{p}(\Omega)$, we denote by $w_{\mu}(\Omega)$ the torsion function in $\Omega$ associated with $\mu$, defined as the (unique) minimizer of the functional

$$
W_{0}^{1, p}(\Omega) \ni v \mapsto \frac{1}{p} \int_{\Omega}|\nabla v|^{p} d \mathcal{L}^{N}+\frac{1}{p} \int_{\Omega}|v|^{p} d \mu-\int_{\Omega} v d \mathcal{L}^{N}
$$

Remark 5.12. The sets $A_{\mu}$ and $\left\{w_{\mu}(\Omega)>0\right\}$ coincide up to sets of null $p$-capacity. 
Proof. From Remark 5.11 we infer that $\operatorname{cap}_{p}\left(\left\{w_{\mu}(\Omega)>0\right\} \backslash A_{\mu}\right)=0$. On the other hand, by the quasiLindelöf property (see Proposition 2.8), there exists a sequence $\left(W_{n}\right)$ of Borel and $p$-finely open subsets of $\Omega$ with $\mu\left(W_{n}\right)<+\infty$ and $\operatorname{cap}_{p}\left(A_{\mu} \backslash \bigcup_{n \in \mathbb{N}} W_{n}\right)=0$. Then we have

$$
\operatorname{cap}_{p}\left(W_{n} \backslash\left\{w_{\mu}(\Omega)>0\right\}\right)=0
$$

by Proposition 5.3 and [20, Theorem 5.1], whence $\operatorname{cap}_{p}\left(A_{\mu} \backslash\left\{w_{\mu}(\Omega)>0\right\}\right)=0$.

If we set

$$
\mathcal{K}^{p}(\Omega)=\left\{v \in W_{0}^{1, p}(\Omega): v \geq 0 \text { a.e. in } \Omega \text { and }-\Delta_{p} v \leq 1 \text { in } W^{-1, p^{\prime}}(\Omega)\right\},
$$

it follows from $\left[20\right.$, Theorem 5.1] that $\mathcal{K}^{p}(\Omega)$, endowed with the weak topology of $W_{0}^{1, p}(\Omega)$, is compact and metrizable. Moreover, again from [20, Theorem 5.1] and from Proposition 5.3, it follows that the map

$$
\begin{array}{cc}
\mathcal{M}_{0}^{p}(\Omega) & \rightarrow \mathcal{K}^{p}(\Omega) \\
\mu & \mapsto w_{\mu}(\Omega)
\end{array}
$$

is bijective. Then $\mathcal{M}_{0}^{p}(\Omega)$ is endowed with the topology that makes such a map a homeomorphism. Therefore, $\mathcal{M}_{0}^{p}(\Omega)$ is a compact and metrizable topological space.

Definition 5.13. If $\Omega$ is a bounded and open subset of $\mathbb{R}^{N}$, a sequence $\left(\mu^{(n)}\right)$ in $\mathcal{M}_{0}^{p}(\Omega)$ is said to be $\gamma^{-\Delta_{p}}$-convergent to $\mu$ if it is convergent to $\mu$ with respect to the topology we have just defined. This means that $\left(w_{\mu^{(n)}}(\Omega)\right)$ is weakly convergent to $w_{\mu}(\Omega)$ in $W_{0}^{1, p}(\Omega)$.

In the following, we will simply write $\gamma$-convergent instead of $\gamma^{-\Delta_{p}}$-convergent. Being a countable product of compact and metrizable topological spaces, also

$$
\prod_{k \in \mathbb{N}} \mathcal{M}_{0}^{p}\left(B_{k}(0)\right)
$$

endowed with the product topology is compact and metrizable.

Proposition 5.14. The map

is injective with closed image.

$$
\begin{aligned}
\mathcal{M}_{0}^{p}\left(\mathbb{R}^{N}\right) & \rightarrow \prod_{k \in \mathbb{N}} \mathcal{M}_{0}^{p}\left(B_{k}(0)\right) \\
\mu & \mapsto \quad\left(\left.\mu\right|_{\mathcal{B}\left(B_{k}(0)\right)}\right)
\end{aligned}
$$

Proof. For every $\mu \in \mathcal{M}_{0}^{p}\left(\mathbb{R}^{N}\right)$ and $A \in \mathcal{B}\left(\mathbb{R}^{N}\right)$ with $A$-quasi open, we have

$$
\mu(A)=\sup _{k} \mu\left(A \cap B_{k}(0)\right) .
$$

Therefore the map is injective.

If $\left(\mu^{(n)}\right)$ is a sequence in $\mathcal{M}_{0}^{p}\left(\mathbb{R}^{N}\right)$ such that $\left(\left.\mu^{(n)}\right|_{\mathcal{B}\left(B_{k}(0)\right)}\right)$ is $\gamma$-convergent to $\nu_{k}$ in $\mathcal{M}_{0}^{p}\left(B_{k}(0)\right)$ for all $k \in \mathbb{N}$, it follows from Proposition 5.3 and [20, Theorem 6.12] that $\left.\nu_{k+1}\right|_{\mathcal{B}\left(B_{k}(0)\right)}=\nu_{k}$. If we set

$$
\mu(A)=\sup _{k} \nu_{k}\left(A \cap B_{k}(0)\right) \quad \text { for all } A \in \mathcal{B}\left(\mathbb{R}^{N}\right) \text { with } A \text {-quasi open }
$$

and we denote by $\mu$ the equivalence class of

$$
\tilde{\mu}(B)=\inf \{\mu(A): A \in \mathcal{B}(\Omega), A \supseteq B \text { and } A \text { is } p \text {-quasi open }\} \quad \text { for all } B \in \mathcal{B}\left(\mathbb{R}^{N}\right),
$$

it is easily seen that $\mu \in \mathcal{M}_{0}^{p}\left(\mathbb{R}^{N}\right)$ and $\left.\mu\right|_{\mathcal{B}\left(B_{k}(0)\right)}=\nu_{k}$ for all $k \in \mathbb{N}$. Therefore the map has closed image.

Then $\mathcal{M}_{0}^{p}\left(\mathbb{R}^{N}\right)$ is endowed with the topology that makes such a map a homeomorphism between $\mathcal{M}_{0}^{p}\left(\mathbb{R}^{N}\right)$ and its image. Therefore, $\mathcal{M}_{0}^{p}\left(\mathbb{R}^{N}\right)$ also is a compact and metrizable topological space. 
Definition 5.15. A sequence $\left(\mu^{(n)}\right)$ in $\mathcal{M}_{0}^{p}\left(\mathbb{R}^{N}\right)$ is said to be locally $\gamma$-convergent to $\mu$ if it is convergent to $\mu$ with respect to the topology we have just defined. Taking into account Proposition 5.3 and [20, Theorem 6.12], this means that $\left(\left.\mu^{(n)}\right|_{\mathcal{B}(\Omega)}\right)$ is $\gamma$-convergent to $\left.\mu\right|_{\mathcal{B}(\Omega)}$ in $\mathcal{M}_{0}^{p}(\Omega)$ for all bounded and open subset $\Omega$ of $\mathbb{R}^{N}$.

In particular, if $A^{(n)}, A$ are $p$-quasi open subsets of $\mathbb{R}^{N}$, the sequence $\left(A^{(n)}\right)$ is said to be locally $\gamma$ convergent to $A$ if $\mu^{(n)}=\infty_{\mathbb{R}^{N} \backslash A^{(n)}}$ is locally $\gamma$-convergent to $\mu=\infty_{\mathbb{R}^{N} \backslash A}$. If $A^{(n)}, A \subseteq \Omega$ for some bounded and open set $\Omega$, then this is equivalent to the classical notion of $\gamma$-convergence of sets.

5.2. Lower estimate and asymptotic equicoercivity for a sequence of functionals. For every $\mu \in \mathcal{M}_{0}^{p}\left(\mathbb{R}^{N}\right)$, we define a first lower semicontinuous and convex functional

$$
f_{\mu}: L_{l o c}^{p}\left(\mathbb{R}^{N}\right) \rightarrow[0,+\infty]
$$

by

$$
f_{\mu}(u)= \begin{cases}\frac{1}{p} \int|\nabla u|^{p} d \mathcal{L}^{N}+\frac{1}{p} \int|u|^{p} d \mu & \text { if } u \in W_{l o c}^{1, p}\left(\mathbb{R}^{N}\right), \\ +\infty & \text { otherwise. }\end{cases}
$$

Proposition 5.16. If $\mu \in \mathcal{M}_{0}^{p}\left(\mathbb{R}^{N}\right)$ and $\left(u^{(n)}\right)$ is a sequence in $W_{\text {loc }}^{1, p}\left(\mathbb{R}^{N}\right) \cap L^{p}\left(\mathbb{R}^{N}, \mu\right)$ satisfying

$$
\sup _{n}\left(\int\left|\nabla u^{(n)}\right|^{p} d \mathcal{L}^{N}+\int\left|u^{(n)}\right|^{p} d \mu\right)<+\infty
$$

and converging to some $u$ in $L_{\text {loc }}^{p}\left(\mathbb{R}^{N}\right)$, then $u \in W_{l o c}^{1, p}\left(\mathbb{R}^{N}\right) \cap L^{p}\left(\mathbb{R}^{N}, \mu\right)$ and $\left(u^{(n)}\right)$ is weakly convergent to $u$ in $L^{p}\left(\mathbb{R}^{N}, \mu\right)$.

Proof. The sequence $\left(\nabla u^{(n)}\right)$ is weakly convergent to $\nabla u$ in $L^{p}\left(\mathbb{R}^{N} ; \mathbb{R}^{N}\right)$ and, up to a subsequence, $\left(u^{(n)}\right)$ is weakly convergent to some $v$ in $L^{p}\left(\mathbb{R}^{N}, \mu\right)$. If we consider

$$
C=\left\{\left(\left.w\right|_{B_{1}(0)}, \nabla w, w\right): w \in W_{l o c}^{1, p}\left(\mathbb{R}^{N}\right) \cap L^{p}\left(\mathbb{R}^{N}, \mu\right) \text { and } \nabla w \in L^{p}\left(\mathbb{R}^{N} ; \mathbb{R}^{N}\right)\right\}
$$

as a convex subset of $L^{p}\left(B_{1}(0)\right) \times L^{p}\left(\mathbb{R}^{N} ; \mathbb{R}^{N}\right) \times L^{p}\left(\mathbb{R}^{N}, \mu\right)$, we have that $\left(\left.u\right|_{B_{1}(0)}, \nabla u, v\right)$ belongs to the weak closure of $C$, as $\left(\left.u^{(n)}\right|_{B_{1}(0)}, \nabla u^{(n)}, u^{(n)}\right) \in C$. Then there exists a sequence $\left(\left.w^{(n)}\right|_{B_{1}(0)}, \nabla w^{(n)}, w^{(n)}\right)$ in $C$ strongly converging to $\left(\left.u\right|_{B_{1}(0)}, \nabla u, v\right)$. Up to a subsequence, $w^{(n)} \rightarrow u$ q.e. in $\mathbb{R}^{N}$, hence $\mu$-a.e. in $\mathbb{R}^{N}$. Then $v=u \mu$-a.e. in $\mathbb{R}^{N}$.

Theorem 5.17. If $\left(\mu^{(n)}\right)$ is locally $\gamma$-convergent to $\mu$ in $\mathcal{M}_{0}^{p}\left(\mathbb{R}^{N}\right)$, then

$$
f_{\mu}(u) \leq\left(\Gamma-\liminf _{n \rightarrow \infty} f_{\mu^{(n)}}\right)(u) \quad \text { for all } u \in L_{l o c}^{p}\left(\mathbb{R}^{N}\right) .
$$

Proof. By Proposition 5.3 we may assume, without loss of generality, that we have chosen for each $\mu^{(n)}$ and for $\mu$ the outer regular representative.

Let $\left(u^{(n)}\right)$ be a sequence converging to $u$ in $L_{l o c}^{p}\left(\mathbb{R}^{N}\right)$ with

$$
\liminf _{n \rightarrow \infty} f_{\mu^{(n)}}\left(u^{(n)}\right)=\left(\Gamma-\liminf _{n \rightarrow \infty} f_{\mu^{(n)}}\right)(u) .
$$

Without loss of generality, we may assume that this value is not $+\infty$. Up to a subsequence, it follows that $u^{(n)}, u \in W_{l o c}^{1, p}\left(\mathbb{R}^{N}\right)$,

$$
\sup _{n}\left(\int\left|\nabla u^{(n)}\right|^{p} d \mathcal{L}^{N}+\int\left|u^{(n)}\right|^{p} d \mu^{(n)}\right)<+\infty
$$

and $\left(\nabla u^{(n)}\right)$ is weakly convergent to $\nabla u$ in $L^{p}\left(\mathbb{R}^{N} ; \mathbb{R}^{N}\right)$.

If we define $\left.b: \mathbb{R}^{N} \rightarrow\right] 0,+\infty[$ by

$$
b(x)=2^{-j}\left(1+\sup _{n} \int_{\{j \leq|x|<j+1\}}\left|u^{(n)}\right|^{p} d \mathcal{L}^{N}\right)^{-1} \quad \text { if } j \geq 0 \text { and } j \leq|x|<j+1,
$$


then $b \in L^{\infty}\left(\mathbb{R}^{N}\right)$, with essinf $b>0$ for all compact subsets $K$ of $\mathbb{R}^{N}$, and $\left|u^{(n)}\right|^{p} b,|u|^{p} b \in L^{1}\left(\mathbb{R}^{N}\right)$ with

$$
\lim _{n \rightarrow \infty} \int\left|u^{(n)}-u\right|^{p} b d \mathcal{L}^{N}=0 .
$$

Now fix $k \in \mathbb{N}$ and define

$$
u_{k}^{(n)}=\underset{v \in W_{l o c}^{1, p}\left(\mathbb{R}^{N}\right)}{\arg \min }\left\{\frac{k}{p} \int\left|u^{(n)}-v\right|^{p} b d \mathcal{L}^{N}+f_{\mu^{(n)}}(v)\right\},
$$

as the above minimization problem admits one and only one minimizer. Then $u_{k}^{(n)} \in L^{p}\left(\mathbb{R}^{N}, \mu^{(n)}\right)$,

$$
\frac{k}{p} \int\left|u^{(n)}-u_{k}^{(n)}\right|^{p} b d \mathcal{L}^{N}+f_{\mu^{(n)}}\left(u_{k}^{(n)}\right) \leq f_{\mu^{(n)}}\left(u^{(n)}\right),
$$

up to a subsequence $\left(u_{k}^{(n)}\right)$ is convergent in $L_{l o c}^{p}\left(\mathbb{R}^{N}\right)$ to some $v_{k} \in W_{l o c}^{1, p}\left(\mathbb{R}^{N}\right)$ and $\left(\nabla u_{k}^{(n)}\right)$ is weakly convergent to $\nabla v_{k}$ in $L^{p}\left(\mathbb{R}^{N} ; \mathbb{R}^{N}\right)$. Moreover

$$
\begin{aligned}
\int\left|\nabla u_{k}^{(n)}\right|^{p-2} \nabla & u_{k}^{(n)} \cdot \nabla v d \mathcal{L}^{N}+\int\left|u_{k}^{(n)}\right|^{p-2} u_{k}^{(n)} v d \mu^{(n)} \\
& =k \int\left|u^{(n)}-u_{k}^{(n)}\right|^{p-2}\left(u^{(n)}-u_{k}^{(n)}\right) v b d \mathcal{L}^{N} \quad \text { for all } v \in W_{c}^{1, p}\left(\mathbb{R}^{N}\right) \cap L^{p}\left(\mathbb{R}^{N}, \mu^{(n)}\right) .
\end{aligned}
$$

Since $\left(\left|u^{(n)}-u_{k}^{(n)}\right|^{p-2}\left(u^{(n)}-u_{k}^{(n)}\right) b\right)$ is strongly convergent to $\left|u-v_{k}\right|^{p-2}\left(u-v_{k}\right) b$ in $L_{l o c}^{p^{\prime}}\left(\mathbb{R}^{N}\right)$, from $[20$, Theorems 6.3 and 6.11] we infer that $v_{k} \in L_{l o c}^{p}\left(\mathbb{R}^{N}, \mu\right)$ and

$$
\lim _{n \rightarrow \infty}\left[\int\left|\nabla u_{k}^{(n)}\right|^{p} \varphi d \mathcal{L}^{n}+\int\left|u_{k}^{(n)}\right|^{p} \varphi d \mu^{(n)}\right]=\int\left|\nabla v_{k}\right|^{p} \varphi d \mathcal{L}^{n}+\int\left|v_{k}\right|^{p} \varphi d \mu
$$

for all $k \in \mathbb{N}$ and $\varphi \in C_{c}\left(\mathbb{R}^{N}\right)$.

In particular, if $\varphi \in C_{c}\left(\mathbb{R}^{N}\right)$ with $0 \leq \varphi \leq 1$, we have

$$
\begin{aligned}
\liminf _{n \rightarrow \infty} f_{\mu^{(n)}}\left(u^{(n)}\right) & \geq \liminf _{n \rightarrow \infty}\left[\frac{k}{p} \int\left|u^{(n)}-u_{k}^{(n)}\right|^{p} b d \mathcal{L}^{N}+f_{\mu^{(n)}}\left(u_{k}^{(n)}\right)\right] \\
& \geq \liminf _{n \rightarrow \infty}\left[\frac{k}{p} \int\left|u^{(n)}-u_{k}^{(n)}\right|^{p} b d \mathcal{L}^{N}+\frac{1}{p} \int\left|\nabla u_{k}^{(n)}\right|^{p} \varphi d \mathcal{L}^{N}\right. \\
& \left.+\frac{1}{p} \int\left|u_{k}^{(n)}\right|^{p} \varphi d \mu^{(n)}\right] \\
& \geq \frac{k}{p} \int\left|u-v_{k}\right|^{p} b d \mathcal{L}^{N}+\frac{1}{p} \int\left|\nabla v_{k}\right|^{p} \varphi d \mathcal{L}^{N}+\frac{1}{p} \int\left|v_{k}\right|^{p} \varphi d \mu .
\end{aligned}
$$

By the arbitrariness of $\varphi$, we infer that $v_{k} \in L^{p}\left(\mathbb{R}^{N}, \mu\right)$ and

$$
\liminf _{n \rightarrow \infty} f_{\mu^{(n)}}\left(u^{(n)}\right) \geq \frac{k}{p} \int\left|u-v_{k}\right|^{p} b d \mathcal{L}^{N}+f_{\mu}\left(v_{k}\right)
$$

for all $k \in \mathbb{N}$, whence

$$
\lim _{k \rightarrow \infty} \int\left|u-v_{k}\right|^{p} b d \mathcal{L}^{N}=0 .
$$

In particular, $\left(v_{k}\right)$ is convergent to $u$ in $L_{l o c}^{p}\left(\mathbb{R}^{N}\right)$. By the lower semicontinuity of $f_{\mu}$ we conclude that

$$
\liminf _{n \rightarrow \infty} f_{\mu^{(n)}}\left(u^{(n)}\right) \geq \liminf _{k \rightarrow \infty} f_{\mu}\left(v_{k}\right) \geq f_{\mu}(u)
$$

and the proof is complete.

Example 5.18. Let $p<N$ and let $\mu^{(n)}=\infty_{\mathbb{R}^{N} \backslash B_{n}(0)}$. Then $\left(\mu^{(n)}\right)$ is locally $\gamma$-convergent to $\mu=0$, but it is false that

$$
f_{\mu}(u)=\left(\Gamma-\lim _{n \rightarrow \infty} f_{\mu^{(n)}}\right)(u) \quad \text { for all } u \in L_{l o c}^{p}\left(\mathbb{R}^{N}\right) .
$$

Actually, if we take $u=1$, we have $f_{\mu}(u)=0$ but it is impossible to find a sequence $\left(u^{(n)}\right)$ converging to 1 in $L_{l o c}^{p}\left(\mathbb{R}^{N}\right)$ with $f_{\mu^{(n)}}\left(u^{(n)}\right) \rightarrow 0$, because each $u^{(n)}$ has compact support, which implies that $\left(u^{(n)}\right)$ is convergent to 0 in $L^{p^{*}}\left(\mathbb{R}^{N}\right)$. 
If $p \geq N$, we will see by Proposition 5.21 and Theorem 5.24 that the assertion is true.

Proposition 5.19. Let $\left(\mu^{(n)}\right)$ be a sequence in $\mathcal{M}_{0}^{p}\left(\mathbb{R}^{N}\right)$ and $\mu \in \mathcal{M}_{0}^{p}\left(\mathbb{R}^{N}\right)$ with $\mu\left(\mathbb{R}^{N}\right)>0$ and

$$
f_{\mu}(u) \leq\left(\Gamma-\liminf _{n \rightarrow \infty} f_{\mu^{(n)}}\right)(u) \quad \text { for all } u \in L_{l o c}^{p}\left(\mathbb{R}^{N}\right) .
$$

Then, for every sequence $\left(u^{(n)}\right)$ in $W_{l o c}^{1, p}\left(\mathbb{R}^{N}\right)$ such that

$$
\sup _{n} f_{\mu^{(n)}}\left(u^{(n)}\right)<+\infty,
$$

there exist $u \in W_{\text {loc }}^{1, p}\left(\mathbb{R}^{N}\right)$ and a subsequence $\left(u^{\left(n_{k}\right)}\right)$ converging to $u$ in $L_{\text {loc }}^{p}\left(\mathbb{R}^{N}\right)$.

Proof. It is enough to prove that

$$
\sup _{n} \int_{B_{1}(0)}\left|u^{(n)}\right|^{p} d \mathcal{L}^{N}<+\infty .
$$

Assume, for the sake of contradiction, that, up to a subsequence, we have

$$
\lim _{n \rightarrow \infty} \int_{B_{1}(0)}\left|u^{(n)}\right|^{p} d \mathcal{L}^{N}=+\infty .
$$

Then a suitably rescaled sequence $\left(v^{(n)}\right)$ satisfies

$$
\int_{B_{1}(0)}\left|v^{(n)}\right|^{p} d \mathcal{L}^{N}=1, \quad \lim _{n \rightarrow \infty}\left(\int\left|\nabla v^{(n)}\right|^{p} d \mathcal{L}^{N}+\int\left|v^{(n)}\right|^{p} d \mu^{(n)}\right)=0 .
$$

It follows that, up to a subsequence, $\left(v^{(n)}\right)$ is convergent to some $v$ in $W_{l o c}^{1, p}\left(\mathbb{R}^{N}\right)$, whence

$$
\int|\nabla v|^{p} d \mathcal{L}^{N}+\int|v|^{p} d \mu=0
$$

so that $v$ is a constant with $v=0$, as $\mu\left(\mathbb{R}^{N}\right)>0$. On the other hand

$$
\int_{B_{1}(0)}|v|^{p} d \mathcal{L}^{N}=1
$$

and a contradiction follows.

5.3. Convergence of functionals. In order to relate the local $\gamma$-convergence of measures in $\mathcal{M}_{0}^{p}\left(\mathbb{R}^{N}\right)$ with the $\Gamma$-convergence of functionals on $\mathbb{R}^{N}$, we need to introduce, roughly speaking, a homogeneous Dirichlet-type condition at infinity.

For every $\mu \in \mathcal{M}_{0}^{p}\left(\mathbb{R}^{N}\right)$, we first define the convex functional

$$
\widetilde{f}_{\mu, 0}: L_{l o c}^{p}\left(\mathbb{R}^{N}\right) \rightarrow[0,+\infty]
$$

by

$$
\widetilde{f}_{\mu, 0}(u)= \begin{cases}\frac{1}{p} \int|\nabla u|^{p} d \mathcal{L}^{N}+\frac{1}{p} \int|u|^{p} d \mu & \text { if } u \in W_{c}^{1, p}\left(\mathbb{R}^{N}\right), \\ +\infty & \text { otherwise }\end{cases}
$$

then we denote by $f_{\mu, 0}$ its lower semicontinuous envelope.

Lemma 5.20. If $p \geq N$, there exists a sequence $\left(\vartheta_{n}\right)$ in $C_{c}^{\infty}\left(\mathbb{R}^{N}\right)$ such that $0 \leq \vartheta_{n} \leq 1$,

$$
\begin{array}{ll}
\lim _{n \rightarrow \infty} \vartheta_{n}=1 & \text { uniformly on compact subsets of } \mathbb{R}^{N}, \\
\lim _{n \rightarrow \infty} \nabla \vartheta_{n}=0 & \text { strongly in } L^{p}\left(\mathbb{R}^{N} ; \mathbb{R}^{N}\right) .
\end{array}
$$

Proof. Consider the space

$$
\mathcal{X}=\left\{u \in W_{l o c}^{1, p+1}\left(\mathbb{R}^{N}\right): \nabla u \in L^{p}\left(\mathbb{R}^{N} ; \mathbb{R}^{N}\right) \cap L^{p+1}\left(\mathbb{R}^{N} ; \mathbb{R}^{N}\right), \int_{B_{1}(0)} u d \mathcal{L}^{N}=0\right\} .
$$

Then $\mathcal{X}$ is a reflexive Banach space, when endowed with the norm

$$
\|u\|=\|\nabla u\|_{p}+\|\nabla u\|_{p+1} .
$$


Let $\hat{\varphi} \in C^{\infty}\left(\mathbb{R}^{N}\right)$ be such that $0 \leq \hat{\varphi} \leq 1, \hat{\varphi}(x)=0$ for $|x| \leq 1$ and $\hat{\varphi}(x)=-1$ for $|x| \geq 2$. Then define $\hat{\varphi}_{n}(x)=\hat{\varphi}(x / n)$ for all $n \geq 1$. Of course $\hat{\varphi}_{n} \in \mathcal{X}$ and it is easily seen that $\left(\hat{\varphi}_{n}\right)$ is weakly convergent to 0 in $\mathcal{X}$ (by the way, strongly if $p>N$ ). Therefore 0 belongs to the weak closure of the convex set

$$
\operatorname{conv}\left\{\hat{\varphi}_{n}: n \in \mathbb{N}\right\} .
$$

Then there exists a sequence $\left(\varphi_{n}\right)$ in such a convex set strongly converging to 0 in $\mathcal{X}$. In particular, each $\varphi_{n}$ satisfies $\varphi_{n}=-1$ outside some compact subset of $\mathbb{R}^{N},\left(\nabla \varphi_{n}\right)$ is strongly convergent to 0 in $L^{p}\left(\mathbb{R}^{N} ; \mathbb{R}^{N}\right)$ and $\left(\varphi_{n}\right)$ is convergent to 0 uniformly on compact subsets of $\mathbb{R}^{N}$, as $p+1>N$.

It follows that $\vartheta_{n}=1+\varphi_{n}$ has the required properties.

Proposition 5.21. If $p<N$, we have

$$
f_{\mu, 0}(u)= \begin{cases}f_{\mu}(u) & \text { if } u \in W_{\text {loc }}^{1, p}\left(\mathbb{R}^{N}\right) \cap L^{p^{*}}\left(\mathbb{R}^{N}\right), \\ +\infty & \text { otherwise. }\end{cases}
$$

If $p \geq N$, we have

$$
f_{\mu, 0}(u)=f_{\mu}(u) \quad \text { for all } u \in L_{l o c}^{p}\left(\mathbb{R}^{N}\right) .
$$

Proof. Since $f_{\mu}$ is lower semicontinuous, we clearly have

$$
f_{\mu}(u) \leq f_{\mu, 0}(u) \leq \widetilde{f}_{\mu, 0}(u) \quad \text { for all } u \in L_{l o c}^{p}\left(\mathbb{R}^{N}\right) .
$$

Assume first that $p<N$. Let $u \in W_{l o c}^{1, p}\left(\mathbb{R}^{N}\right) \cap L^{p^{*}}\left(\mathbb{R}^{N}\right)$ and let $\vartheta \in C^{\infty}(\mathbb{R})$ be such that $0 \leq \vartheta \leq 1$, $\vartheta^{\prime} \leq 0, \vartheta(s)=1$ for $s \leq 1$ and $\vartheta(s)=0$ for $s \geq 2$. If we set $\vartheta_{n}(x)=\vartheta(|x| / n)$, we have $0 \leq \vartheta_{n} \leq \vartheta_{n+1} \leq 1$, $\vartheta_{n} u \in W_{c}^{1, p}\left(\mathbb{R}^{N}\right)$ and $\left(\vartheta_{n} u\right)$ is convergent to $u$ in $L_{l o c}^{p}\left(\mathbb{R}^{N}\right)$. We also have

$$
f_{\mu, 0}\left(\vartheta_{n} u\right)=\frac{1}{p} \int\left|\vartheta_{n} \nabla u+u \nabla \vartheta_{n}\right|^{p} d \mathcal{L}^{N}+\frac{1}{p} \int\left|\vartheta_{n} u\right|^{p} d \mu .
$$

It is easily seen that $\left(\nabla \vartheta_{n}\right)$ is bounded in $L^{N}\left(\mathbb{R}^{N} ; \mathbb{R}^{N}\right)$ and convergent to 0 a.e. in $\mathbb{R}^{N}$. Moreover, for every $\varepsilon>0$ there exists $C_{\varepsilon}>0$ such that

$$
|u(x) \xi| \leq C_{\varepsilon}|u(x)|^{p^{*} / p}+\varepsilon|\xi|^{N / p} \quad \text { for a.a. } x \in \mathbb{R}^{n} \text { and all } \xi \in \mathbb{R}^{N} .
$$

It follows (see e.g. [21, Lemma 4.2]) that $\left(u \nabla \vartheta_{n}\right)$ is strongly convergent to 0 in $L^{p}\left(\mathbb{R}^{N} ; \mathbb{R}^{N}\right)$. By the lower semicontinuity of $f_{\mu, 0}$ we infer that

$$
f_{\mu, 0}(u) \leq \lim _{n \rightarrow \infty} f_{\mu, 0}\left(\vartheta_{n} u\right)=\frac{1}{p} \int|\nabla u|^{p} d \mathcal{L}^{N}+\frac{1}{p} \int|u|^{p} d \mu=f_{\mu}(u) .
$$

Now it remains only to show that $f_{\mu, 0}(u)=+\infty$ whenever $u \in W_{l o c}^{1, p}\left(\mathbb{R}^{N}\right) \backslash L^{p^{*}}\left(\mathbb{R}^{N}\right)$. Assume, for the sake of contradiction, that $f_{\mu, 0}(u)<+\infty$ and let $\left(u_{n}\right)$ be a sequence converging to $u$ in $L_{l o c}^{p}\left(\mathbb{R}^{N}\right)$ with $\widetilde{f}_{\mu, 0}(u)\left(u_{n}\right) \rightarrow f_{\mu, 0}(u)$, whence $u_{n} \in W_{c}^{1, p}\left(\mathbb{R}^{N}\right)$ eventually as $n \rightarrow \infty$. Since $\left(\nabla u_{n}\right)$ is bounded in $L^{p}\left(\mathbb{R}^{N} ; \mathbb{R}^{N}\right)$, we have that $\left(u_{n}\right)$ is bounded in $L^{p^{*}}\left(\mathbb{R}^{N}\right)$. Therefore, $u \in L^{p^{*}}\left(\mathbb{R}^{N}\right)$ and a contradiction follows.

If $p \geq N$, let $u \in W_{l o c}^{1, p}\left(\mathbb{R}^{N}\right) \cap L^{p}\left(\mathbb{R}^{N}, \mu\right)$ with $\nabla u \in L^{p}\left(\mathbb{R}^{N} ; \mathbb{R}^{N}\right)$, let $\left(\vartheta_{n}\right)$ be a sequence as in Lemma 5.20 and let $\left(c_{n}\right)$ be sequence of positive numbers increasing to $+\infty$ such that $\left\|c_{n} \nabla \vartheta_{n}\right\|_{p} \rightarrow 0$. If we define

$$
u_{n}=\min \left\{\max \left\{u,-c_{n} \vartheta_{n}\right\}, c_{n} \vartheta_{n}\right\},
$$

we have

$$
\left|\nabla u_{n}\right|^{p} \leq|\nabla u|^{p}+\left|c_{n} \nabla \vartheta_{n}\right|^{p} \quad \text { a.e. in } \mathbb{R}^{N} .
$$

Then we have that $\left(u_{n}\right)$ is convergent to $u$ in $L_{l o c}^{p}\left(\mathbb{R}^{N}\right)$ with $u_{n} \in W_{c}^{1, p}\left(\mathbb{R}^{N}\right)$ and

$$
f_{\mu, 0}(u) \leq \lim _{n \rightarrow \infty} f_{\mu, 0}\left(u_{n}\right)=\frac{1}{p} \int|\nabla u|^{p} d \mathcal{L}^{N}+\frac{1}{p} \int|u|^{p} d \mu=f_{\mu}(u),
$$

whence the assertion.

Before dealing with the main result of this subsection, we need the following.

Proposition 5.22. Let $\mu, \nu \in \mathcal{M}_{0}^{p}\left(\mathbb{R}^{N}\right)$ be such that $f_{\mu, 0} \leq f_{\nu, 0}$. Then $\mu \leq \nu$. 
Proof. We have

$$
\int|u|^{p} d \mu \leq \int|u|^{p} d \nu \quad \text { for all } u \in W_{c}^{1, p}\left(\mathbb{R}^{N}\right),
$$

whence

$$
\int|u|^{p} d \mu \leq \int|u|^{p} d \nu \quad \text { for all } u \in W_{l o c}^{1, p}\left(\mathbb{R}^{N}\right) .
$$

For every Borel and $p$-quasi open subset $A$ of $\Omega$, there exists $u \in W_{l o c}^{1, p}\left(\mathbb{R}^{N}\right)$ such that $A=\{u>0\}$ by Proposition 2.8. It follows

$$
\int_{\Omega}\left(\min \left\{k u^{+}, 1\right\}\right)^{p} d \mu \leq \int_{\Omega}\left(\min \left\{k u^{+}, 1\right\}\right)^{p} d \nu \quad \text { for all } k \in \mathbb{N},
$$

whence $\mu(A) \leq \nu(A)$ going to the limit as $k \rightarrow \infty$.

Corollary 5.23. Let $\mu, \nu \in \mathcal{M}_{0}^{p}\left(\mathbb{R}^{N}\right)$ be such that $f_{\mu} \leq f_{\nu}$. Then $\mu \leq \nu$.

Proof. It follows from Propositions 5.21 and 5.22.

The main purpose of this subsection is to show that a sequence of measures $\left(\mu^{(n)}\right)$ is convergent to $\mu$ in $\mathcal{M}_{0}^{p}\left(\mathbb{R}^{N}\right)$ if and only if $f_{\mu^{(n)}, 0}$ is $\Gamma$-convergent to $f_{\mu, 0}$ in $L_{l o c}^{p}\left(\mathbb{R}^{N}\right)$. In the case $p=2$ a similar result was obtained by Bucur in [7, Appendix]; our more general case requires a more involved proof.

Theorem 5.24. A sequence $\left(\mu^{(n)}\right)$ is locally $\gamma$-convergent to $\mu$ in $\mathcal{M}_{0}^{p}\left(\mathbb{R}^{N}\right)$ if and only if

$$
f_{\mu, 0}(u)=\left(\Gamma-\lim _{n \rightarrow \infty} f_{\mu^{(n)}, 0}\right)(u) \quad \text { for all } u \in L_{l o c}^{p}\left(\mathbb{R}^{N}\right) .
$$

Proof. Again, by Proposition 5.3 we may assume, without loss of generality, that we have chosen for each $\mu^{(n)}$ and for $\mu$ the outer regular representative.

Assume first that $\left(\mu^{(n)}\right)$ is locally $\gamma$-convergent to $\mu$.

Step 1. $\Gamma$-liminf inequality. By Proposition 5.21 and Theorem 5.17, we have to treat only the case $p<N$. We take a sequence $\left(u^{(n)}\right)$ converging to $u$ in $L_{l o c}^{p}\left(\mathbb{R}^{N}\right)$ with

$$
\liminf _{n \rightarrow \infty} f_{\mu^{(n)}}\left(u^{(n)}\right)=\left(\Gamma-\liminf _{n \rightarrow \infty} f_{\mu^{(n)}, 0}\right)(u)
$$

and, without loss of generality, we may assume that $u^{(n)} \in W_{l o c}^{1, p}\left(\mathbb{R}^{N}\right) \cap L^{p^{*}}\left(\mathbb{R}^{N}\right)$ with

$$
\sup _{n}\left(\int\left|\nabla u^{(n)}\right|^{p} d \mathcal{L}^{N}+\int\left|u^{(n)}\right|^{p} d \mu^{(n)}\right)<+\infty .
$$

Since $\left(\nabla u^{(n)}\right)$ is bounded in $L^{p}\left(\mathbb{R}^{N} ; \mathbb{R}^{N}\right)$, we have that $\left(u^{(n)}\right)$ is bounded in $L^{p^{*}}\left(\mathbb{R}^{N}\right)$. Therefore $u \in$ $L^{p^{*}}\left(\mathbb{R}^{N}\right)$ and the assertion follows again from Proposition 5.21 and Theorem 5.17.

Step 2. $\Gamma$-limsup inequality. Let $u \in L_{l o c}^{p}\left(\mathbb{R}^{N}\right)$ with $f_{\mu, 0}(u)<+\infty$, let $\beta>f_{\mu, 0}(u)$ and let $U$ be an open neighborhood of $u$ in $L_{l o c}^{p}\left(\mathbb{R}^{N}\right)$. Let $z \in U \cap W_{c}^{1, p}\left(\mathbb{R}^{N}\right)$ with $f_{\mu, 0}(z) \leq \beta$ and let $R>0$ be such that $z=0$ a.e. in $\mathbb{R}^{N} \backslash B_{R}(0)$. For every $k \in \mathbb{N}$, define

$$
u_{k}=\underset{v \in W_{0}^{1, p}\left(B_{R}(0)\right)}{\arg \min }\left\{\frac{k}{p} \int_{B_{R}(0)}|z-v|^{p} d \mathcal{L}^{N}+f_{\mu, 0}(v)\right\},
$$

as the above minimization problem admits one and only one minimizer. Then, testing with $v=z$ we obtain the upper bound

$$
\frac{k}{p} \int_{B_{R}(0)}\left|z-u_{k}\right|^{p} d \mathcal{L}^{N}+f_{\mu, 0}\left(u_{k}\right) \leq f_{\mu, 0}(z) \leq \beta,
$$

for all $k \in \mathbb{N}$. Thus $\left(u_{k}\right)$ is convergent to $z$ in $L^{p}\left(B_{R}(0)\right)$. Let us fix $k$ large enough to have $u_{k} \in U$.

Then $u_{k} \in W_{0}^{1, p}\left(B_{R}(0)\right) \cap L^{p}\left(B_{R}(0), \mu\right)$ and the Euler-Lagrange equation for the minimization problem defining $u_{k}$ yields

$$
\begin{aligned}
& \int_{B_{R}(0)}\left|\nabla u_{k}\right|^{p-2} \nabla u_{k} \cdot \nabla v d \mathcal{L}^{N}+\int_{B_{R}(0)}\left|u_{k}\right|^{p-2} u_{k} v d \mu \\
&=k \int_{B_{R}(0)}\left|z-u_{k}\right|^{p-2}\left(z-u_{k}\right) v d \mathcal{L}^{N} \quad \text { for all } v \in W_{0}^{1, p}\left(B_{R}(0)\right) \cap L^{p}\left(B_{R}(0), \mu\right) .
\end{aligned}
$$


Now, for every $n \in \mathbb{N}$, let

$$
u_{k}^{(n)}=\underset{v \in W_{0}^{1, p}\left(B_{R}(0)\right)}{\arg \min }\left\{f_{\mu^{(n)}, 0}(v)-k \int_{B_{R}(0)}\left|z-u_{k}\right|^{p-2}\left(z-u_{k}\right) v d \mathcal{L}^{N}\right\},
$$

as again this problem has one and only one minimizer. Then we have $u_{k}^{(n)} \in W_{0}^{1, p}\left(B_{R}(0)\right) \cap L^{p}\left(B_{R}(0), \mu^{(n)}\right)$ and

$$
\begin{aligned}
& \int_{B_{R}(0)}\left|\nabla u_{k}^{(n)}\right|^{p-2} \nabla u_{k}^{(n)} \cdot \nabla v d \mathcal{L}^{N}+\int_{B_{R}(0)}\left|u_{k}^{(n)}\right|^{p-2} u_{k}^{(n)} v d \mu^{(n)} \\
&=k \int_{B_{R}(0)}\left|z-u_{k}\right|^{p-2}\left(z-u_{k}\right) v d \mathcal{L}^{N} \quad \text { for all } v \in W_{0}^{1, p}\left(B_{R}(0)\right) \cap L^{p}\left(B_{R}(0), \mu^{(n)}\right) .
\end{aligned}
$$

From [20, Theorem 6.3] we infer that $\left(u_{k}^{(n)}\right)$ is weakly convergent to $u_{k}$ in $W_{0}^{1, p}\left(B_{R}(0)\right)$. In particular, we have $u_{k}^{(n)} \in U$ eventually as $n \rightarrow \infty$. Moreover, it is

$$
\begin{aligned}
f_{\mu^{(n), 0}}\left(u_{k}^{(n)}\right) & =\frac{1}{p} \int_{B_{R}(0)}\left|\nabla u_{k}^{(n)}\right|^{p} d \mathcal{L}^{N}+\frac{1}{p} \int_{B_{R}(0)}\left|u_{k}^{(n)}\right|^{p} d \mu^{(n)} \\
& =\frac{k}{p} \int_{B_{R}(0)}\left|z-u_{k}\right|^{p-2}\left(z-u_{k}\right) u_{k}^{(n)} d \mathcal{L}^{N} \\
f_{\mu, 0}\left(u_{k}\right) & =\frac{1}{p} \int_{B_{R}(0)}\left|\nabla u_{k}\right|^{p} d \mathcal{L}^{N}+\frac{1}{p} \int_{B_{R}(0)}\left|u_{k}\right|^{p} d \mu \\
& =\frac{k}{p} \int_{B_{R}(0)}\left|z-u_{k}\right|^{p-2}\left(z-u_{k}\right) u_{k} d \mathcal{L}^{N} .
\end{aligned}
$$

Therefore, having in mind the topological definition of $\Gamma$-limsup, we obtain

$$
\limsup _{n \rightarrow \infty}\left(\inf _{v \in U} f_{\mu^{(n)}, 0}(v)\right) \leq \lim _{n \rightarrow \infty} f_{\mu^{(n)}, 0}\left(u_{k}^{(n)}\right)=f_{\mu, 0}\left(u_{k}\right) \leq \beta
$$

and the assertion follows from the arbitrariness of $\beta$ and $U$.

Assume now that

$$
f_{\mu, 0}(u)=\left(\Gamma-\lim _{n \rightarrow \infty} f_{\mu^{(n)}, 0}\right)(u) \quad \text { for all } u \in L_{l o c}^{p}\left(\mathbb{R}^{N}\right) .
$$

Up to a subsequence, $\left(\mu^{(n)}\right)$ is locally $\gamma$-convergent to some $\nu$ in $\mathcal{M}_{0}^{p}\left(\mathbb{R}^{N}\right)$. By the previous step, we infer that

$$
f_{\nu, 0}(u)=\left(\Gamma-\lim _{n \rightarrow \infty} f_{\mu^{(n)}, 0}\right)(u) \quad \text { for all } u \in L_{l o c}^{p}\left(\mathbb{R}^{N}\right),
$$

whence $f_{\nu, 0}=f_{\mu, 0}$. By Proposition 5.22 we have $\nu=\mu$ and the assertion follows.

We conclude the section by highlighting some further consequences of the local $\gamma$-convergence.

Corollary 5.25. Let $\left(\mu^{(n)}\right)$ be locally $\gamma$-convergent to $\mu$ and $\left(\nu^{(n)}\right)$ be locally $\gamma$-convergent to $\nu$ in $\mathcal{M}_{0}^{p}\left(\mathbb{R}^{N}\right)$ with $\mu^{(n)} \leq \nu^{(n)}$ for all $n \in \mathbb{N}$.

Then $\mu \leq \nu$.

Proof. It follows from Theorem 5.24 and Proposition 5.22.

Corollary 5.26. If $\left(\mu^{(n)}\right)$ is locally $\gamma$-convergent to $\mu$ in $\mathcal{M}_{0}^{p}\left(\mathbb{R}^{N}\right)$, then

$$
\begin{aligned}
\mathcal{L}^{N}\left(A_{\mu}\right) & \leq \liminf _{n \rightarrow \infty} \mathcal{L}^{N}\left(A_{\mu^{(n)}}\right), \\
\int \Psi\left(V_{\mu}\right) d \mathcal{L}^{N} & \leq \liminf _{n \rightarrow \infty} \int \Psi\left(V_{\mu^{(n)}}\right) d \mathcal{L}^{N},
\end{aligned}
$$

whenever $\Psi:[0,+\infty] \rightarrow[0,+\infty]$ is a strictly decreasing and continuous function such that there exists $\alpha>1$ with $\left\{s \mapsto \Psi^{-1}\left(s^{\alpha}\right)\right\}$ convex on $\left\{s \geq 0: s^{\alpha} \in \Psi([0,+\infty])\right\}$. 
Proof. If $\Omega$ is a bounded and open subset of $\mathbb{R}^{N}$ and $\left(\mu^{(n)}\right)$ is $\gamma$-convergent to $\mu$ in $\mathcal{M}_{0}^{p}(\Omega)$, then

$$
\mathcal{L}^{N}\left(A_{\mu}\right) \leq \liminf _{n \rightarrow \infty} \mathcal{L}^{N}\left(A_{\mu^{(n)}}\right),
$$

as

$$
\mathcal{L}^{N}\left(A_{\nu}\right)=\sup _{k \in \mathbb{N}} \int_{\Omega} \min \left\{k w_{\nu}(\Omega), 1\right\} d \mathcal{L}^{N}
$$

for all $\nu \in \mathcal{M}_{0}^{p}(\Omega)$ by Remark 5.12.

On the other hand, for every $\nu \in \mathcal{M}_{0}^{p}\left(\mathbb{R}^{N}\right)$ we have

$$
\mathcal{L}^{N}\left(A_{\nu}\right)=\sup _{k \in \mathbb{N}} \mathcal{L}^{N}\left(A_{\nu} \cap B_{k}(0)\right)=\sup _{k \in \mathbb{N}} \mathcal{L}^{N}\left(A_{\left.\nu\right|_{\mathcal{B}\left(B_{k}(0)\right)}}\right)
$$

and the first assertion follows.

When dealing with the second assertion, we follow an argument inspired by [12, Theorem 4.1]. Without loss of generality, we assume that

$$
\sup _{n} \int \Psi\left(V_{\mu^{(n)}}\right) d \mathcal{L}^{N}<+\infty
$$

and we set $v_{n}=\left(\Psi\left(V_{\mu(n)}\right)\right)^{1 / \alpha}$, so that $\left(v_{n}\right)$ is a bounded sequence in $L^{\alpha}\left(\mathbb{R}^{N}\right)$, thus (up to subsequences) weakly convergent to some $v$ in $L^{\alpha}\left(\mathbb{R}^{N}\right)$. On the other hand, by Theorem 5.24 , for every $u \in W_{c}^{1, p}\left(\mathbb{R}^{N}\right)$ there exists a sequence $\left(u^{(n)}\right)$ in $W_{l o c}^{1, p}\left(\mathbb{R}^{N}\right)$ converging to $u$ in $L_{l o c}^{p}\left(\mathbb{R}^{N}\right)$ such that

$$
\int|\nabla u|^{p} d \mathcal{L}^{N}+\int|u|^{p} d \mu=\limsup _{n \rightarrow \infty}\left(\int\left|\nabla u^{(n)}\right|^{p} d \mathcal{L}^{N}+\int\left|u^{(n)}\right|^{p} d \mu^{(n)}\right) .
$$

Combining assertion $(a)$ of Proposition 5.9 with the strong-weak lower semicontinuity theorem of [32], we infer that

$$
\begin{aligned}
\int|\nabla u|^{p} d \mathcal{L}^{N}+\int|u|^{p} d \mu & \geq \limsup _{n \rightarrow \infty}\left(\int\left|\nabla u^{(n)}\right|^{p} d \mathcal{L}^{N}+\int\left|u^{(n)}\right|^{p} V_{\mu(n)} d \mathcal{L}^{N}\right) \\
& =\limsup _{n \rightarrow \infty}\left(\int\left|\nabla u^{(n)}\right|^{p} d \mathcal{L}^{N}+\int\left|u^{(n)}\right|^{p} \Psi^{-1}\left(v_{n}^{\alpha}\right) d \mathcal{L}^{N}\right) \\
& \geq \int|\nabla u|^{p} d \mathcal{L}^{N}+\int|u|^{p} \Psi^{-1}\left(v^{\alpha}\right) d \mathcal{L}^{N},
\end{aligned}
$$

as the function $\left\{s \mapsto \Psi^{-1}\left(s^{\alpha}\right)\right\}$ is convex.

By Proposition 5.22, we infer

$$
\mu(A) \geq \int_{A} \Psi^{-1}\left(v^{\alpha}\right) d \mathcal{L}^{N} \quad \text { for all } A \in \mathcal{B}\left(\mathbb{R}^{N}\right) \text { with } A \text { p-quasi open },
$$

whence $V_{\mu} \geq \Psi^{-1}\left(v^{\alpha}\right) \mathcal{L}^{N}$-a.e. in $\mathbb{R}^{N}$ by $(b)$ and $(d)$ of Proposition 5.9.

Since $\Psi$ is strictly decreasing, we infer that $\Psi\left(V_{\mu}\right) \leq v^{\alpha} \mathcal{L}^{N}$-a.e. in $\mathbb{R}^{N}$, whence

$$
\int \Psi\left(V_{\mu}\right) d \mathcal{L}^{N} \leq \int v^{\alpha} d \mathcal{L}^{N} \leq \liminf _{n \rightarrow \infty} \int v_{n}^{\alpha} d \mathcal{L}^{N}=\liminf _{n \rightarrow \infty} \int \Psi\left(V_{\mu(n)}\right) d \mathcal{L}^{N}
$$

and the second assertion also follows.

\section{TOWARdS VARIATIONAL EIGENVALUES FOR SIGN-CHANGING CAPACITARY MEASURES}

Let $\mu, \nu_{1}, \nu_{2} \in \mathcal{M}_{0}^{p}\left(\mathbb{R}^{N}\right)$. In this section we introduce the candidate "variational eigenvalues" for the problem

$$
\left\{\begin{array}{l}
-\Delta_{p} u+|u|^{p-2} u \mu=\lambda|u|^{p-2} u\left(\nu_{1}-\nu_{2}\right) \quad \text { in } \mathbb{R}^{N}, \\
\int|u|^{p} d \nu_{2}<\int|u|^{p} d \nu_{1},
\end{array}\right.
$$

and prove some basic properties. 
Consider an index $i$ as in Section 3 and the related families $\mathcal{K}_{m}$ and $\mathcal{K}_{m}^{\text {fin }}$ with respect to the metrizable and locally convex topological vector space $\mathcal{X}=L_{l o c}^{p}\left(\mathbb{R}^{N}\right)$. Let $f_{\mu, 0}: L_{l o c}^{p}\left(\mathbb{R}^{N}\right) \rightarrow[0,+\infty]$ be the functional introduced in Section 5 and define $g_{1}, g_{2}, R: L_{l o c}^{p}\left(\mathbb{R}^{N}\right) \rightarrow[0,+\infty]$ by

$$
\begin{gathered}
g_{j}(u)= \begin{cases}\frac{1}{p} \int|u|^{p} d \nu_{j} & \text { if } u \in W_{l o c}^{1, p}\left(\mathbb{R}^{N}\right), \\
+\infty & \text { otherwise, }\end{cases} \\
R(u)= \begin{cases}f_{\mu, 0}(u) & \text { if } 1+g_{2}(u) \leq g_{1}(u)<+\infty, \\
+\infty & \text { otherwise. }\end{cases}
\end{gathered}
$$

Then, for every integer $m \geq 1$, set

$$
\lambda_{m}^{p}\left(\mu, \nu_{1}, \nu_{2}\right)=\inf _{K \in \mathcal{K}_{m}} \sup _{u \in K} R(u)
$$

Remark 6.1. It is immediate from the definition to note that, if $\mu_{1}, \mu_{2} \in \mathcal{M}_{0}^{p}\left(\mathbb{R}^{N}\right)$ with $\mu_{1} \leq \mu_{2}$, then

$$
\lambda_{m}^{p}\left(\mu_{1}, \nu_{1}, \nu_{2}\right) \leq \lambda_{m}^{p}\left(\mu_{2}, \nu_{1}, \nu_{2}\right) \quad \text { for all } \nu_{1}, \nu_{2} \in \mathcal{M}_{0}^{p}\left(\mathbb{R}^{N}\right) \text { and } m \geq 1 \text {. }
$$

Proposition 6.2. For every $u \in W_{l o c}^{1, p}\left(\mathbb{R}^{N}\right)$ with

$$
f_{\mu, 0}(u)<+\infty, \quad \int|u|^{p} d \nu_{1}<+\infty, \quad \int|u|^{p} d \nu_{2}<+\infty,
$$

there exists a sequence $\left(u_{n}\right)$ in $W_{c}^{1, p}\left(\mathbb{R}^{N}\right) \cap L^{p}\left(\mathbb{R}^{N}, \mu\right) \cap L^{p}\left(\mathbb{R}^{N}, \nu_{1}\right) \cap L^{p}\left(\mathbb{R}^{N}, \nu_{2}\right)$ converging to $u$ in $L_{\text {loc }}^{p}\left(\mathbb{R}^{N}\right)$ such that

$$
\lim _{n \rightarrow \infty}\left(\int\left|\nabla u_{n}-\nabla u\right|^{p} d \mathcal{L}^{N}+\int\left|u_{n}-u\right|^{p} d \mu+\int\left|u_{n}-u\right|^{p} d \nu_{1}+\int\left|u_{n}-u\right|^{p} d \nu_{2}\right)=0 .
$$

Proof. By Proposition 5.21 we also have $f_{\mu+\nu_{1}+\nu_{2}, 0}(u)<+\infty$. Therefore, there exists a sequence $\left(u_{n}\right)$ in $W_{c}^{1, p}\left(\mathbb{R}^{N}\right) \cap L^{p}\left(\mathbb{R}^{N}, \mu\right) \cap L^{p}\left(\mathbb{R}^{N}, \nu_{1}\right) \cap L^{p}\left(\mathbb{R}^{N}, \nu_{2}\right)$ converging to $u$ in $L_{\text {loc }}^{p}\left(\mathbb{R}^{N}\right)$ such that

$$
\begin{aligned}
\lim _{n \rightarrow \infty}\left(\int\left|\nabla u_{n}\right|^{p} d \mathcal{L}^{N}+\int\left|u_{n}\right|^{p} d \mu+\int\left|u_{n}\right|^{p} d \nu_{1}+\int\left|u_{n}\right|^{p} d \nu_{2}\right) & \\
& =\int|\nabla u|^{p} d \mathcal{L}^{N}+\int|u|^{p} d \mu+\int|u|^{p} d \nu_{1}+\int|u|^{p} d \nu_{2}
\end{aligned}
$$

Taking into account Proposition 5.16, we have that $\left(\nabla u_{n}\right)$ is weakly convergent to $\nabla u$ in $L^{p}\left(\mathbb{R}^{N} ; \mathbb{R}^{N}\right)$ and $\left(u_{n}\right)$ is weakly convergent to $u$ in $L^{p}\left(\mathbb{R}^{N}, \mu\right), L^{p}\left(\mathbb{R}^{N}, \nu_{1}\right)$ and $L^{p}\left(\mathbb{R}^{N}, \nu_{2}\right)$ with

$$
\begin{aligned}
\lim _{n \rightarrow \infty} \int\left|\nabla u_{n}\right|^{p} d \mathcal{L}^{N}=\int|\nabla u|^{p} d \mathcal{L}^{N}, \quad \lim _{n \rightarrow \infty} \int\left|u_{n}\right|^{p} d \mu=\int|u|^{p} d \mu \\
\lim _{n \rightarrow \infty} \int\left|u_{n}\right|^{p} d \nu_{1}=\int|u|^{p} d \nu_{1}, \quad \lim _{n \rightarrow \infty} \int\left|u_{n}\right|^{p} d \nu_{2}=\int|u|^{p} d \nu_{2}
\end{aligned}
$$

Then the assertion follows.

Proposition 6.3. The following facts hold:

(a) if

$$
\left\{u \in W_{c}^{1, p}\left(\mathbb{R}^{N}\right): \int|u|^{p} d \mu<+\infty, \int|u|^{p} d \nu_{2}<\int|u|^{p} d \nu_{1}<+\infty\right\}=\emptyset,
$$

(b) if

then we have $\lambda_{m}^{p}\left(\mu, \nu_{1}, \nu_{2}\right)=+\infty$ for all $m \geq 1$;

$$
\left\{u \in W_{c}^{1, p}\left(\mathbb{R}^{N}\right): \int|u|^{p} d \mu<+\infty, \int|u|^{p} d \nu_{2}<\int|u|^{p} d \nu_{1}<+\infty\right\} \neq \emptyset,
$$

then we have $\lambda_{1}^{p}\left(\mu, \nu_{1}, \nu_{2}\right)<+\infty$; 
(c) if

$$
\begin{aligned}
\left\{u \in W_{c}^{1, p}\left(\mathbb{R}^{N}\right): \int|u|^{p} d \mu<+\infty, \int|u|^{p} d \nu_{2}<\right. & \int|u|^{p} d \nu_{1}<+\infty, \\
& \left.\lim _{r \rightarrow 0} \int_{B_{r}(x)}|u|^{p} d \nu_{1}=0 \quad \text { for all } x \in \mathbb{R}^{N}\right\} \neq \emptyset,
\end{aligned}
$$

then we have $\lambda_{m}^{p}\left(\mu, \nu_{1}, \nu_{2}\right)<+\infty$ for all $m \geq 1$.

Proof.

(a) From Proposition 6.2 it follows that $R(u)=+\infty$ for all $u \in L_{l o c}^{p}\left(\mathbb{R}^{N}\right)$, whence the assertion.

(b) If $u \in W_{c}^{1, p}\left(\mathbb{R}^{N}\right)$ satisfies

$$
\int|u|^{p} d \mu<+\infty, \int|u|^{p} d \nu_{2}<\int|u|^{p} d \nu_{1}<+\infty,
$$

it is easily seen that $R(t u)<+\infty$ for some $t>0$, whence the assertion.

(c) Let $u \in W_{c}^{1, p}\left(\mathbb{R}^{N}\right)$ with

$$
\int|u|^{p} d \mu<+\infty, \int|u|^{p} d \nu_{2}<\int|u|^{p} d \nu_{1}<+\infty, \lim _{r \rightarrow 0} \int_{B_{r}(x)}|u|^{p} d \nu_{1}=0 \quad \text { for all } x \in \mathbb{R}^{N}
$$

and let us choose a representative for $u, \nu_{1}$ and $\nu_{2}$.

By substituting $u$ with

$$
u_{k}=\left(|u|-\frac{1}{k}\right)^{+}
$$

with $k$ large enough, we may assume that $u \geq 0$ a.e. in $\mathbb{R}^{N}$ and that

$$
\nu_{1}\left(\left\{x \in \mathbb{R}^{N}: u(x)>0\right\}\right)<+\infty, \quad \nu_{2}\left(\left\{x \in \mathbb{R}^{N}: u(x)>0\right\}\right)<+\infty .
$$

If we set

$$
\hat{\nu}(B)=\nu_{1}\left(B \cap\left\{x \in \mathbb{R}^{N}: u(x)>0\right\}\right)+\nu_{2}\left(B \cap\left\{x \in \mathbb{R}^{N}: u(x)>0\right\}\right) \quad \text { for all } B \in \mathcal{B}\left(\mathbb{R}^{N}\right),
$$

we have that $\hat{\nu}$ is a positive Radon measure on $\mathbb{R}^{N}$ and there exist two Borel functions $\eta_{1}, \eta_{2}: \mathbb{R}^{N} \rightarrow[0,1]$ such that

$$
\int_{B} \eta_{j} d \hat{\nu}=\nu_{j}\left(B \cap\left\{x \in \mathbb{R}^{N}: u(x)>0\right\}\right) \quad \text { for all } B \in \mathcal{B}\left(\mathbb{R}^{N}\right),
$$

whence

$$
\int u^{p}\left(\eta_{1}-\eta_{2}\right) d \hat{\nu}>0
$$

We have

$$
\lim _{r \rightarrow 0^{+}} \int_{B_{r}(x)} u^{p}\left(\eta_{1}-\eta_{2}\right) d \hat{\nu} \leq \lim _{r \rightarrow 0^{+}} \int_{B_{r}(x)} u^{p} d \nu_{1}=0 \quad \text { for all } x \in \mathbb{R}^{N} .
$$

Therefore, if $x \in \mathbb{R}^{N}$ is a Lebesgue point of $u^{p}\left(\eta_{1}-\eta_{2}\right)$ with respect to $\hat{\nu}$ such that $u(x)^{p}\left(\eta_{1}(x)-\eta_{2}(x)\right)>0$ (see [1, Corollary 2.23]), we have $\hat{\nu}(\{x\})=0$. Then, for every $m \geq 1$, we can find $m$ Lebesgue points $x_{1}, \ldots, x_{m}$ of $u^{p}\left(\eta_{1}-\eta_{2}\right)$ with respect to $\hat{\nu}$ such that

$$
u^{p}\left(x_{j}\right)\left(\eta_{1}\left(x_{j}\right)-\eta_{2}\left(x_{j}\right)\right)>0 \quad \text { for all } j=1, \ldots, m .
$$

Let $r>0$ be such that $B_{r}\left(x_{i}\right) \cap B_{r}\left(x_{j}\right)=\emptyset$ whenever $i \neq j$ and such that

$$
\int_{B_{r}\left(x_{j}\right)} u^{p}\left(\eta_{1}-\eta_{2}\right) d \hat{\nu}>0 \quad \text { for all } j=1, \ldots, m .
$$

For every $j=1, \ldots, m$, let $\vartheta_{j} \in C_{c}^{\infty}\left(B_{r}\left(x_{j}\right)\right)$ be such that

$$
\int\left(\vartheta_{j} u\right)^{p}\left(\eta_{1}-\eta_{2}\right) d \hat{\nu}>0 \quad \text { for all } j=1, \ldots, m .
$$


If we set

$$
\begin{gathered}
\pi(\xi)=\sum_{j=1}^{m} \xi_{j} \vartheta_{j} u, \\
K=\left\{\frac{\pi(\xi)}{\left(\int|\pi(\xi)|^{p} d \nu_{1}-\int|\pi(\xi)|^{p} d \nu_{2}\right)^{1 / p}}: \xi \in S^{m-1}\right\},
\end{gathered}
$$

we have $K \in \mathcal{K}_{m}$ and $\sup _{u \in K} R(u)<+\infty$, whence $\lambda_{m}^{p}\left(\mu, \nu_{1}, \nu_{2}\right)<+\infty$.

Example 6.4. Let $p>N, \mu=\infty_{\mathbb{R}^{N} \backslash B_{1}(0)}, \nu_{1}=\delta_{0}$ and $\nu_{2}=0$. Since

$$
\left\{u \in W_{0}^{1, p}\left(B_{1}\right): u(0) \neq 0\right\}=\left\{u \in W_{0}^{1, p}\left(B_{1}\right): u(0)<0\right\} \cup\left\{u \in W_{0}^{1, p}\left(B_{1}\right): u(0)>0\right\},
$$

we have $i(K)=1$ for all nonempty, compact and symmetric subset $K$ of $L_{l o c}^{p}\left(\mathbb{R}^{N}\right) \backslash\{0\}$ with

$$
\sup _{u \in K} R(u)<+\infty \text {. }
$$

Therefore, it follows that

$$
\lambda_{m}^{p}\left(\mu, \nu_{1}, \nu_{2}\right)=+\infty \text { for all } m \geq 2 .
$$

By the way, a direct computation shows that

$$
\lambda_{1}^{p}\left(\mu, \nu_{1}, \nu_{2}\right)=\frac{1}{w(0)^{p-1}},
$$

where $w \in W_{0}^{1, p}\left(B_{1}\right)$ satisfies $-\Delta_{p} w=\delta_{0}$.

Proposition 6.5. Assume one of the following conditions:

(a) if $\left(u_{n}\right)$ is a sequence in $W_{c}^{1, p}\left(\mathbb{R}^{N}\right)$ satisfying

$$
\sup _{n}\left(\int\left|\nabla u_{n}\right|^{p} d \mathcal{L}^{N}+\int\left|u_{n}\right|^{p} d \mu+\int\left|u_{n}\right|^{p} d \nu_{1}+\int\left|u_{n}\right|^{p} d \nu_{2}\right)<+\infty
$$

and converging in $L_{l o c}^{p}\left(\mathbb{R}^{N}\right)$ to some $u \in W_{l o c}^{1, p}\left(\mathbb{R}^{N}\right)$, then

$$
\lim _{n \rightarrow \infty} \int\left|u_{n}\right|^{p} d \nu_{1}=\int|u|^{p} d \nu_{1}
$$

(b) we have $\nu_{2}=0$.

Then, for every integer $m \geq 1$, we have

$$
\lambda_{m}^{p}\left(\mu, \nu_{1}, \nu_{2}\right)=\inf _{K \in \mathcal{K}_{m}^{f i n}} \sup _{u \in K} R(u) .
$$

Proof. We aim to apply Theorem 3.5 and Remark 3.6. Actually, assumption (b) of Remark 3.6 follows from assumption $(a)$ and Proposition 6.2, while assumption $(c)$ of Remark 3.6 follows from Proposition 5.16 and assumption $(b)$.

Proposition 6.6. If we set $\mu^{(n)}=\mu+\infty_{\mathbb{R}^{N} \backslash B_{n}(0)}$ and define $R^{(n)}$ accordingly, then we have

$$
\inf _{K \in \mathcal{K}_{m}^{f i n}} \sup _{u \in K} R(u)=\lim _{n \rightarrow \infty}\left(\inf _{K \in \mathcal{K}_{m}^{f i n}} \sup _{u \in K} R^{(n)}(u)\right) \quad \text { for all } m \geq 1 .
$$

If either assumption (a) or assumption (b) of Proposition 6.5 is satisfied, then we also have

$$
\lambda_{m}^{p}\left(\mu, \nu_{1}, \nu_{2}\right)=\lim _{n \rightarrow \infty} \lambda_{m}^{p}\left(\mu^{(n)}, \nu_{1}, \nu_{2}\right) \quad \text { for all } m \geq 1 .
$$


Proof. Of course, we have

To prove that

$$
\inf _{K \in \mathcal{K}_{m}^{f i n}} \sup _{u \in K} R(u) \leq \liminf _{n \rightarrow \infty}\left(\inf _{K \in \mathcal{K}_{m}^{f i n}} \sup _{u \in K} R^{(n)}(u)\right) .
$$

$$
\inf _{K \in \mathcal{K}_{m}^{f i n}} \sup _{u \in K} R(u) \geq \limsup _{n \rightarrow \infty}\left(\inf _{K \in \mathcal{K}_{m}^{f i n}} \sup _{u \in K} R^{(n)}(u)\right),
$$

we aim to apply Theorem 3.4. Assumption $(a)$ is clearly satisfied, while assumption $(b)$ follows from Proposition 6.2 and assumption $(c)$ follows from Proposition 5.16. Therefore, the first claim is proved.

Then we also have

$$
\begin{gathered}
\lambda_{m}^{p}\left(\mu, \nu_{1}, \nu_{2}\right) \leq \liminf _{n \rightarrow \infty} \lambda_{m}^{p}\left(\mu^{(n)}, \nu_{1}, \nu_{2}\right), \\
\limsup _{n \rightarrow \infty} \lambda_{m}^{p}\left(\mu^{(n)}, \nu_{1}, \nu_{2}\right) \leq \inf _{K \in \mathcal{K}_{m}^{\text {fin }}} \sup _{u \in K} R(u) .
\end{gathered}
$$

By Proposition 6.5 the second assertion follows.

\section{Semicontinuity PRoperties of INF-SUP VAlUES OF MEASURES}

Throughout this section, we consider three sequences $\left(\mu^{(n)}\right),\left(\nu_{1}^{(n)}\right),\left(\nu_{2}^{(n)}\right)$ in $\mathcal{M}_{0}^{p}\left(\mathbb{R}^{N}\right)$, three measures $\mu, \nu_{1}, \nu_{2} \in \mathcal{M}_{0}^{p}\left(\mathbb{R}^{N}\right)$, an index $i$ as in Section 3 and the related inf-sup values $\lambda_{m}^{p}\left(\mu^{(n)}, \nu_{1}^{(n)}, \nu_{2}^{(n)}\right)$ and $\lambda_{m}^{p}\left(\mu, \nu_{1}, \nu_{2}\right)$ defined in Section 6 with respect to the metrizable and locally convex topological vector space $\mathcal{X}=L_{l o c}^{p}\left(\mathbb{R}^{N}\right)$.

We also consider the functionals $f_{\mu^{(n)}, 0}, f_{\mu, 0}$ defined in Section 5 and we define

$$
g_{1}^{(n)}, g_{2}^{(n)}, R^{(n)}: L_{l o c}^{p}\left(\mathbb{R}^{N}\right) \rightarrow[0,+\infty]
$$

by

$$
\begin{aligned}
g_{j}^{(n)}(u) & = \begin{cases}\frac{1}{p} \int|u|^{p} d \nu_{j}^{(n)}, & \text { if } u \in W_{l o c}^{1, p}\left(\mathbb{R}^{N}\right), \\
+\infty & \text { otherwise },\end{cases} \\
R^{(n)}(u) & = \begin{cases}f_{\mu^{(n)}, 0}(u) & \text { if } 1+g_{2}^{(n)}(u) \leq g_{1}^{(n)}(u)<+\infty, \\
+\infty & \text { otherwise, }\end{cases}
\end{aligned}
$$

and

$$
g_{1}, g_{2}, R: L_{l o c}^{p}\left(\mathbb{R}^{N}\right) \rightarrow[0,+\infty]
$$

in the analogous way with $\mu, \nu_{1}, \nu_{2}$ instead of $\mu^{(n)}, \nu_{1}^{(n)}, \nu_{2}^{(n)}$.

7.1. Lower semicontinuity of inf-sup values of measures. Throughout this subsection we assume that:

(is) if $\left(n_{k}\right)$ is a strictly increasing sequence in $\mathbb{N}$ and $\left(u^{(k)}\right)$ is a sequence in $W_{c}^{1, p}\left(\mathbb{R}^{N}\right)$ satisfying

$$
\sup _{k}\left(\int\left|\nabla u^{(k)}\right|^{p} d \mathcal{L}^{N}+\int\left|u^{(k)}\right|^{p} d \mu^{\left(n_{k}\right)}+\int\left|u^{(k)}\right|^{p} d \nu_{1}^{\left(n_{k}\right)}+\int\left|u^{(k)}\right|^{p} d \nu_{2}^{\left(n_{k}\right)}\right)<+\infty
$$

and converging in $L_{l o c}^{p}\left(\mathbb{R}^{N}\right)$ to some $u \in W_{l o c}^{1, p}\left(\mathbb{R}^{N}\right)$, then

$$
\limsup _{k \rightarrow \infty} \int\left|u^{(k)}\right|^{p} d \nu_{1}^{\left(n_{k}\right)} \leq \int|u|^{p} d \nu_{1}
$$

(iis) if $p \geq N$, we do not have $\mu\left(\mathbb{R}^{N}\right)=0$ and $\nu_{2}\left(\mathbb{R}^{N}\right) \leq \nu_{1}\left(\mathbb{R}^{N}\right)<+\infty$;

(iiis) we have

$$
\begin{array}{r}
f_{\mu, 0}+\lambda g_{2}+I_{\left\{g_{1}<+\infty\right\}} \leq\left(\Gamma-\liminf _{n \rightarrow \infty}\left(f_{\mu^{(n)}, 0}+\lambda g_{2}^{(n)}+I_{\left\{g_{1}^{(n)}<+\infty\right\}}\right)\right)(u) \\
\quad \text { for all } \lambda>0 \text { and } u \in L_{l o c}^{p}\left(\mathbb{R}^{N}\right) .
\end{array}
$$


Lemma 7.1. If $\left(n_{k}\right)$ is a strictly increasing sequence in $\mathbb{N}$ and $\left(u^{(k)}\right)$ is a sequence in $W_{\text {loc }}^{1, p}\left(\mathbb{R}^{N}\right)$ satisfying

$$
\sup _{k} f_{\mu^{\left(n_{k}\right), 0}}\left(u^{(k)}\right)<+\infty, \sup _{k} \int\left|u^{(k)}\right|^{p} d \nu_{2}^{\left(n_{k}\right)}<+\infty, \sup _{k} \int\left|u^{(k)}\right|^{p} d \nu_{1}^{\left(n_{k}\right)}<+\infty
$$

and converging in $L_{l o c}^{p}\left(\mathbb{R}^{N}\right)$ to some $u \in W_{l o c}^{1, p}\left(\mathbb{R}^{N}\right)$, then

$$
\begin{aligned}
\liminf _{k \rightarrow \infty} f_{\mu^{\left(n_{k}\right)}, 0}\left(u^{(k)}\right) & \geq f_{\mu, 0}(u), \\
\liminf _{k \rightarrow \infty} \int\left|u^{(k)}\right|^{p} d \nu_{2}^{\left(n_{k}\right)} & \geq \int|u|^{p} d \nu_{2}, \\
\limsup _{k \rightarrow \infty} \int\left|u^{(k)}\right|^{p} d \nu_{1}^{\left(n_{k}\right)} & \leq \int|u|^{p} d \nu_{1}<+\infty .
\end{aligned}
$$

Proof. Let $d$ be a compatible distance in $L_{l o c}^{p}\left(\mathbb{R}^{N}\right)$. By Proposition 6.2, for every $k \in \mathbb{N}$ there exists $v^{(k)} \in W_{c}^{1, p}\left(\mathbb{R}^{N}\right)$ such that

$$
\begin{aligned}
\left(d\left(v^{(k)}, u^{(k)}\right)+\int\left|\nabla v^{(k)}-\nabla u^{(k)}\right|^{p} d \mathcal{L}^{N}+\right. & \int\left|v^{(k)}-u^{(k)}\right|^{p} d \mu^{\left(n_{k}\right)} \\
& \left.+\int\left|v^{(k)}-u^{(k)}\right|^{p} d \nu_{1}^{\left(n_{k}\right)}+\int\left|v^{(k)}-u^{(k)}\right|^{p} d \nu_{2}^{\left(n_{k}\right)}\right)<\frac{1}{k} .
\end{aligned}
$$

From assumption $(i s)$ we infer that

$$
\limsup _{k \rightarrow \infty} \int\left|u^{(k)}\right|^{p} d \nu_{1}^{\left(n_{k}\right)}=\limsup _{k \rightarrow \infty} \int\left|v^{(k)}\right|^{p} d \nu_{1}^{\left(n_{k}\right)} \leq \int|u|^{p} d \nu_{1} .
$$

On the other hand, by assumption (iiis) we have

$$
\int|u|^{p} d \nu_{1}<+\infty
$$

and, for every $\lambda>0$,

$$
\begin{aligned}
f_{\mu, 0}(u) & \leq f_{\mu, 0}(u)+\lambda \int|u|^{p} d \nu_{2} \\
& \leq \liminf _{k \rightarrow \infty}\left(f_{\mu^{\left(n_{k}\right), 0}}\left(u^{(k)}\right)+\lambda \int\left|u^{(k)}\right|^{p} d \nu_{2}^{\left(n_{k}\right)}\right) \\
& \leq \liminf _{k \rightarrow \infty} f_{\mu^{\left(n_{k}\right), 0}}\left(u^{(k)}\right)+\lambda \limsup _{k \rightarrow \infty} \int\left|u^{(k)}\right|^{p} d \nu_{2}^{\left(n_{k}\right)}, \\
\int|u|^{p} d \nu_{2} & \leq \frac{1}{\lambda}\left(f_{\mu, 0}(u)+\lambda \int|u|^{p} d \nu_{2}\right) \\
& \leq \frac{1}{\lambda} \liminf _{k \rightarrow \infty}\left(f_{\mu^{\left(n_{k}\right)}, 0}\left(u^{(k)}\right)+\lambda \int\left|u^{(k)}\right|^{p} d \nu_{2}^{\left(n_{k}\right)}\right) \\
& \leq \frac{1}{\lambda} \limsup _{k \rightarrow \infty} f_{\mu^{\left(n_{k}\right)}, 0}\left(u^{(k)}\right)+\liminf _{k \rightarrow \infty} \int\left|u^{(k)}\right|^{p} d \nu_{2}^{\left(n_{k}\right)} .
\end{aligned}
$$

By the arbitrariness of $\lambda$ the assertion follows.

Proposition 7.2. If $\left(n_{k}\right)$ is a strictly increasing sequence in $\mathbb{N}$ and $\left(u^{(k)}\right)$ is a sequence in $W_{\text {loc }}^{1, p}\left(\mathbb{R}^{N}\right)$ satisfying

$$
\sup _{k} f_{\mu^{\left(n_{k}\right), 0}}\left(u^{(k)}\right)<+\infty, \quad \sup _{k} \int\left|u^{(k)}\right|^{p} d \nu_{2}^{\left(n_{k}\right)}<+\infty, \quad \int\left|u^{(k)}\right|^{p} d \nu_{1}^{\left(n_{k}\right)}<+\infty \quad \text { for all } k \in \mathbb{N},
$$


then there exists a subsequence $\left(u^{\left(k_{j}\right)}\right)$ converging in $L_{l o c}^{p}\left(\mathbb{R}^{N}\right)$ to some $u \in W_{\text {loc }}^{1, p}\left(\mathbb{R}^{N}\right)$ with

$$
\begin{aligned}
\liminf _{j \rightarrow \infty} f_{\mu^{\left(n_{k_{j}}\right), 0}}\left(u^{\left(k_{j}\right)}\right) & \geq f_{\mu, 0}(u), \\
\liminf _{j \rightarrow \infty} \int\left|u^{\left(k_{j}\right)}\right|^{p} d \nu_{2}^{\left(n_{k_{j}}\right)} & \geq \int|u|^{p} d \nu_{2}, \\
\limsup _{j \rightarrow \infty} \int\left|u^{\left(k_{j}\right)}\right|^{p} d \nu_{1}^{\left(n_{k_{j}}\right)} & \leq \int|u|^{p} d \nu_{1}<+\infty .
\end{aligned}
$$

Proof. Consider first the particular case in which

$$
\sup _{k} f_{\mu^{\left(n_{k}\right)}, 0}\left(u^{(k)}\right)<+\infty, \sup _{k} \int\left|u^{(k)}\right|^{p} d \nu_{2}^{\left(n_{k}\right)}<+\infty, \sup _{k} \int\left|u^{(k)}\right|^{p} d \nu_{1}^{\left(n_{k}\right)}<+\infty .
$$

By Lemma 7.1 it is enough to prove that

$$
\liminf _{k \rightarrow \infty} \int_{B_{1}(0)}\left|u^{(k)}\right|^{p} d \mathcal{L}^{N}<+\infty .
$$

If $p<N$, this fact follows from the boundedness of $\left(\nabla u_{k}\right)$ in $L^{p}\left(\mathbb{R}^{N} ; \mathbb{R}^{N}\right)$. If $p \geq N$, assume for the sake of contradiction that

$$
\lim _{k \rightarrow \infty} \int_{B_{1}(0)}\left|u^{(k)}\right|^{p} d \mathcal{L}^{N}=+\infty .
$$

Then a suitably rescaled sequence $\left(v^{(k)}\right)$ satisfies

$$
\begin{aligned}
& \lim _{k \rightarrow \infty} f_{\mu^{\left(n_{k}\right)}, 0}\left(v^{(k)}\right)=\lim _{k \rightarrow \infty} \int\left|v^{(k)}\right|^{p} d \nu_{2}^{\left(n_{k}\right)}=\lim _{k \rightarrow \infty} \int\left|v^{(k)}\right|^{p} d \nu_{1}^{\left(n_{k}\right)}=0, \\
& \qquad \int_{B_{1}(0)}\left|v^{(k)}\right|^{p} d \mathcal{L}^{N}=1 \quad \text { for all } k \in \mathbb{N} .
\end{aligned}
$$

Up to a subsequence, $\left(v^{(k)}\right)$ is convergent in $W_{l o c}^{1, p}\left(\mathbb{R}^{N}\right)$ to some $v$ satisfying, by Lemma 7.1,

$$
\lim _{n \rightarrow \infty} f_{\mu, 0}(v)=\int|v|^{p} d \nu_{2}=0, \quad \int|v|^{p} d \nu_{1}<+\infty, \quad \int_{B_{1}(0)}|v|^{p} d \mathcal{L}^{N}=1 .
$$

Therefore $v$ is a nonzero constant and $\mu\left(\mathbb{R}^{N}\right)=\nu_{2}\left(\mathbb{R}^{N}\right)=0$, while $\nu_{1}\left(\mathbb{R}^{N}\right)<+\infty$. This fact contradicts assumption (iis).

Now let us treat the general case and suppose, for the sake of contradiction, that up to a subsequence

$$
\lim _{k \rightarrow \infty} \int\left|u^{(k)}\right|^{p} d \nu_{1}^{\left(n_{k}\right)}=+\infty .
$$

Then a suitably rescaled sequence $\left(v^{(k)}\right)$ satisfies

$$
\lim _{k \rightarrow \infty} f_{\mu^{\left(n_{k}\right)}, 0}\left(v^{(k)}\right)=0, \lim _{k \rightarrow \infty} \int\left|v^{(k)}\right|^{p} d \nu_{2}^{\left(n_{k}\right)}=0, \int\left|v^{(k)}\right|^{p} d \nu_{1}^{\left(n_{k}\right)}=1 \quad \text { for all } k \in \mathbb{N} .
$$

By the previous step, up to a subsequence $\left(v^{(k)}\right)$ is convergent in $L_{l o c}^{p}\left(\mathbb{R}^{N}\right)$ to some $v \in W_{\text {loc }}^{1, p}\left(\mathbb{R}^{N}\right)$ such that

$$
f_{\mu, 0}(v)=0, \quad \int|v|^{p} d \nu_{2}=0, \quad 1 \leq \int|v|^{p} d \nu_{1}<+\infty .
$$

It follows that $v$ is a nonzero constant and that $\mu\left(\mathbb{R}^{N}\right)=\nu_{2}\left(\mathbb{R}^{N}\right)=0$, while $\nu_{1}\left(\mathbb{R}^{N}\right)<+\infty$. If $p<N$, this is a contradiction, as $v \in L^{p^{*}}\left(\mathbb{R}^{N}\right)$. If $p \geq N$, a contradiction follows from assumption (iis).

Theorem 7.3. For every integer $m \geq 1$, we have

$$
\lambda_{m}^{p}\left(\mu, \nu_{1}, \nu_{2}\right) \leq \liminf _{n \rightarrow \infty} \lambda_{m}^{p}\left(\mu^{(n)}, \nu_{1}^{(n)}, \nu_{2}^{(n)}\right) .
$$

Proof. We aim to apply Corollary 3.2 with $\mathcal{X}=L_{\text {loc }}^{p}\left(\mathbb{R}^{N}\right)$. Assumption $(a)$ of Corollary 3.2 is obviously satisfied, while assumption (b) of Corollary 3.2 is just assumption (iiis) and assumption (c) of Corollary 3.2 follows from Proposition 7.2. 
Finally, if $u \in W_{l o c}^{1, p}\left(\mathbb{R}^{N}\right) \backslash\{0\}$ and $f_{\mu, 0}(u)=0$, we infer that $u$ is constant, $p \geq N, \mu\left(\mathbb{R}^{N}\right)=0$ and we cannot have

$$
\int|u|^{p} d \nu_{2} \leq \int|u|^{p} d \nu_{1}<+\infty
$$

by assumption $($ iis $)$. Therefore assumption $(d)$ of Corollary 3.2 is satisfied and the assertion follows.

7.2. Upper semicontinuity of inf-sup values of measures. Throughout this subsection we assume that:

(ivs) if $\left(n_{k}\right)$ is a strictly increasing sequence in $\mathbb{N}$ and $\left(u^{(k)}\right)$ a sequence converging to $u$ in $L_{\text {loc }}^{p}\left(\mathbb{R}^{N}\right) \backslash\{0\}$ with

$$
\begin{aligned}
\sup _{k} f_{\mu^{\left(n_{k}\right), 0}}\left(u^{(k)}\right)<+\infty, & \sup _{k} \int\left|u^{(k)}\right|^{p} d \nu_{1}^{\left(n_{k}\right)}<+\infty, \\
& \int\left|u^{(k)}\right|^{p} d \nu_{2}^{\left(n_{k}\right)}<\int\left|u^{(k)}\right|^{p} d \nu_{1}^{\left(n_{k}\right)} \quad \text { for all } k \in \mathbb{N},
\end{aligned}
$$

then

$$
\int|u|^{p} d \nu_{1} \leq \liminf _{k \rightarrow \infty} \int\left|u^{(k)}\right| d \nu_{1}^{\left(n_{k}\right)}
$$

(vs) we have

$$
\begin{array}{r}
f_{\mu, 0}+\lambda g_{2}+I_{\left\{g_{1}<+\infty\right\}} \geq\left(\Gamma-\limsup _{n \rightarrow \infty}\left(f_{\mu^{(n)}, 0}+\lambda g_{2}^{(n)}+I_{\left\{g_{1}^{(n)}<+\infty\right\}}\right)\right)(u) \\
\quad \text { for all } \lambda>0 \text { and } u \in L_{l o c}^{p}\left(\mathbb{R}^{N}\right) .
\end{array}
$$

Theorem 7.4. Assume one of the following conditions:

(a) if $\left(u_{n}\right)$ is a sequence in $W_{c}^{1, p}\left(\mathbb{R}^{N}\right)$ satisfying

$$
\sup _{n}\left(\int\left|\nabla u_{n}\right|^{p} d \mathcal{L}^{N}+\int\left|u_{n}\right|^{p} d \mu+\int\left|u_{n}\right|^{p} d \nu_{1}+\int\left|u_{n}\right|^{p} d \nu_{2}\right)<+\infty
$$

and converging in $L_{l o c}^{p}\left(\mathbb{R}^{N}\right)$ to some $u \in W_{l o c}^{1, p}\left(\mathbb{R}^{N}\right)$, then

$$
\lim _{n \rightarrow \infty} \int\left|u_{n}\right|^{p} d \nu_{1}=\int|u|^{p} d \nu_{1}
$$

(b) we have $\nu_{2}=0$.

Then, for every integer $m \geq 1$, we have

$$
\lambda_{m}^{p}\left(\mu, \nu_{1}, \nu_{2}\right) \geq \limsup _{n \rightarrow \infty} \lambda_{m}^{p}\left(\mu^{(n)}, \nu_{1}^{(n)}, \nu_{2}^{(n)}\right) .
$$

Proof. First of all we claim that, by Theorem 3.4, we have

$$
\inf _{K \in \mathcal{K}_{m}^{f i n}} \sup _{u \in K} R(u) \geq \limsup _{n \rightarrow \infty}\left(\inf _{K \in \mathcal{K}_{m}^{f i n}} \sup _{u \in K} R^{(n)}(u)\right) .
$$

Actually, assumption ( $a$ ) of Theorem 3.4 is obviously satisfied, while assumption (b) is assumption (vs) and assumption $(c)$ is implied by assumption (ivs).

A fortiori we have

$$
\inf _{K \in \mathcal{K}_{m}^{f i n}} \sup _{u \in K} R(u) \geq \limsup _{n \rightarrow \infty} \lambda_{m}^{p}\left(\mu^{(n)}, \nu_{1}^{(n)}, \nu_{2}^{(n)}\right)
$$

and the assertion follows from Proposition 6.5. 


\section{EXISTENCE OF NONLINEAR EIGENVECTORS FOR SIGN-CHANGING CAPACITARY MEASURES}

Let $\mu, \nu_{1}, \nu_{2} \in \mathcal{M}_{0}^{p}\left(\mathbb{R}^{N}\right)$. In this section we want to show that, under suitable assumptions, the inf-sup values $\lambda_{m}^{p}\left(\mu, \nu_{1}, \nu_{2}\right)$ introduced in Section 6 are true eigenvalues of the problem

$$
\left\{\begin{array}{l}
-\Delta_{p} u+|u|^{p-2} u \mu=\lambda|u|^{p-2} u\left(\nu_{1}-\nu_{2}\right) \quad \text { in } \mathbb{R}^{N} \\
\int|u|^{p} d \nu_{2}<\int|u|^{p} d \nu_{1},
\end{array}\right.
$$

with corresponding eigenvectors. To this aim, we will relate the inf-sup values $\lambda_{m}^{p}\left(\mu, \nu_{1}, \nu_{2}\right)$ with the inf-sup values $\hat{\lambda}_{m}^{p}\left(\mu, \nu_{1}, \nu_{2}\right)$ defined in a functional setting where standard variational methods apply.

Throughout this section we assume that:

$$
\begin{aligned}
& \text { if }\left(u_{n}\right) \text { is a sequence in } W_{c}^{1, p}\left(\mathbb{R}^{N}\right) \text { satisfying } \\
& \sup _{n}\left(\int\left|\nabla u_{n}\right|^{p} d \mathcal{L}^{N}+\int\left|u_{n}\right|^{p} d \mu+\int\left|u_{n}\right|^{p} d \nu_{1}+\int\left|u_{n}\right|^{p} d \nu_{2}\right)<+\infty
\end{aligned}
$$

and converging in $L_{\text {loc }}^{p}\left(\mathbb{R}^{N}\right)$ to some $u \in W_{\text {loc }}^{1, p}\left(\mathbb{R}^{N}\right)$, then

$$
\lim _{n \rightarrow \infty} \int\left|u_{n}\right|^{p} d \nu_{1}=\int|u|^{p} d \nu_{1}
$$

(ii) if $p \geq N$, we do not have $\mu\left(\mathbb{R}^{N}\right)=0$ and $\nu_{2}\left(\mathbb{R}^{N}\right) \leq \nu_{1}\left(\mathbb{R}^{N}\right)<+\infty$.

Taking into account Proposition 5.16, these assumptions turn out to be hypotheses (is) and (iis) of Section 7 , in the case in which $\mu^{(n)}=\mu, \nu_{1}^{(n)}=\nu_{1}$ and $\nu_{2}^{(n)}=\nu_{2}$.

Proposition 8.1. If $\left(u_{n}\right)$ is a sequence in $W_{l o c}^{1, p}\left(\mathbb{R}^{N}\right)$ satisfying

$$
\sup _{n} f_{\mu, 0}\left(u_{n}\right)<+\infty, \sup _{n} \int\left|u_{n}\right|^{p} d \nu_{2}<+\infty, \int\left|u_{n}\right|^{p} d \nu_{1}<+\infty \quad \text { for all } n \in \mathbb{N},
$$

then there exists a subsequence $\left(u^{\left(n_{j}\right)}\right)$ converging in $L_{\text {loc }}^{p}\left(\mathbb{R}^{N}\right)$ to some $u \in W_{\text {loc }}^{1, p}\left(\mathbb{R}^{N}\right)$ with

$$
\begin{aligned}
\liminf _{j \rightarrow \infty} f_{\mu, 0}\left(u_{n_{j}}\right) & \geq f_{\mu, 0}(u) \\
\liminf _{j \rightarrow \infty} \int\left|u^{\left(n_{j}\right)}\right|^{p} d \nu_{2} & \geq \int|u|^{p} d \nu_{2}, \\
\lim _{j \rightarrow \infty} \int\left|u^{\left(n_{j}\right)}\right|^{p} d \nu_{1} & =\int|u|^{p} d \nu_{1}<+\infty .
\end{aligned}
$$

Proof. Taking into account Proposition 5.16, it is a particular case of Proposition 7.2.

Now we set

$$
X=\left\{u \in W_{l o c}^{1, p}\left(\mathbb{R}^{N}\right) \cap L^{p}\left(\mathbb{R}^{N}, \nu_{1}\right) \cap L^{p}\left(\mathbb{R}^{N}, \nu_{2}\right): f_{\mu, 0}(u)<+\infty\right\} .
$$

Proposition 8.2. We have that $X$ is a vector subspace of $W_{l o c}^{1, p}\left(\mathbb{R}^{N}\right)$ and

$$
\|u\|:=\left(\int|\nabla u|^{p} d \mathcal{L}^{N}+\int|u|^{p} d \mu+\int|u|^{p} d \nu_{1}+\int|u|^{p} d \nu_{2}\right)^{1 / p}
$$

is a norm on $X$ which makes $X$ a uniformly convex Banach space.

Moreover, $X \cap W_{c}^{1, p}\left(\mathbb{R}^{N}\right)$ is dense in $X$ and the linear maps

$$
\begin{array}{cccccc}
X & \longrightarrow & L_{l o c}^{p}\left(\mathbb{R}^{N}\right) & X & \longrightarrow & L^{p}\left(\mathbb{R}^{N}, \nu_{1}\right) \\
u & \mapsto & u & u & \mapsto & u
\end{array}
$$

are completely continuous.

Proof. It is easily seen that $X$ is a vector subspace of $W_{l o c}^{1, p}\left(\mathbb{R}^{N}\right)$ and that $\|u\|$ is a norm in $X$. In particular, assumption (ii) guarantees that $\|u\|=0$ only if $u=0$.

Of course

$$
\begin{array}{ccc}
X & \longrightarrow & L^{p}\left(\mathbb{R}^{N} ; \mathbb{R}^{N}\right) \times L^{p}\left(\mathbb{R}^{N}, \mu\right) \times L^{p}\left(\mathbb{R}^{N}, \nu_{1}\right) \times L^{p}\left(\mathbb{R}^{N}, \nu_{2}\right) \\
u & \mapsto & (\nabla u, u, u, u)
\end{array}
$$


is a linear isometry. We claim that its image is closed. Actually, if $\left(u_{n}\right)$ is a sequence in $X$ such that $\left(\left(\nabla u_{n}, u_{n}, u_{n}, u_{n}\right)\right)$ is convergent to $\left(U, v_{1}, v_{2}, v_{3}\right)$, from Propositions 8.1 and 5.16 we infer that, up to a subsequence, $\left(u_{n}\right)$ is convergent in $L_{l o c}^{p}\left(\mathbb{R}^{N}\right)$ to some $u \in X$ with $(\nabla u, u, u, u)=\left(U, v_{1}, v_{2}, v_{3}\right)$ and the claim follows.

Therefore, $X$ is a uniformly convex Banach space. By Proposition 6.2 we have that $X \cap W_{c}^{1, p}\left(\mathbb{R}^{N}\right)$ is dense in $X$, while the linear maps

$$
\begin{array}{cccccc}
X & \longrightarrow & L_{l o c}^{p}\left(\mathbb{R}^{N}\right) & X & \longrightarrow & L^{p}\left(\mathbb{R}^{N}, \nu_{1}\right) \\
u & \mapsto & u & u & \mapsto & u
\end{array}
$$

are completely continuous by Propositions 8.1 and 5.16 .

Remark 8.3. We will see that in $X$ standard variational methods apply. On the other hand $X$ depends on $\mu, \nu_{1}$ and $\nu_{2}$, while $L_{l o c}^{p}\left(\mathbb{R}^{N}\right)$ is a fixed space, hence more suitable for $\Gamma$-convergence.

If $\mu=\infty_{\mathbb{R}^{N} \backslash A}$, where $A$ is $p$-quasi open, $\nu_{1}=\mathcal{L}^{N}$ and $\nu_{2}=0$, then $X=W_{0}^{1, p}(A)$ endowed with the usual structure of uniformly convex Banach space.

We also define $g_{1}, g_{2}: L_{l o c}^{p}\left(\mathbb{R}^{N}\right) \rightarrow[0,+\infty]$ by

$$
g_{j}(u)= \begin{cases}\frac{1}{p} \int|u|^{p} d \nu_{j} & \text { if } u \in W_{l o c}^{1, p}\left(\mathbb{R}^{N}\right), \\ +\infty & \text { otherwise }\end{cases}
$$

and set

$$
\varphi=\left.f_{\mu, 0}\right|_{X}, \psi_{1}=\left.g_{1}\right|_{X}, \psi_{2}=\left.g_{2}\right|_{X}, \widehat{M}=\left\{u \in X: \psi_{1}(u)-\psi_{2}(u)=1\right\} .
$$

Of course, $\varphi, \psi_{1}$ and $\psi_{2}$ are even, convex, positively homogeneous of degree $p$ and of class $C^{1}$. According to Section 4 , we denote by $\widehat{\mathcal{K}}_{m}$ the family of nonempty, compact and symmetric subsets $K$ of $\widehat{M}$ (with respect to the topology of $X)$ such that $i(K) \geq m$ and we set

$$
\hat{\lambda}_{m}^{p}\left(\mu, \nu_{1}, \nu_{2}\right)=\inf _{K \in \widehat{\mathcal{K}}_{m}} \max _{u \in K} \varphi(u),
$$

where we agree that $\hat{\lambda}_{m}^{p}\left(\mu, \nu_{1}, \nu_{2}\right)=+\infty$ if there is no $K$ included in $\widehat{M}$ with $i(K) \geq m$.

Theorem 8.4. For every integer $m \geq 1$, we have

$$
\hat{\lambda}_{m}^{p}\left(\mu, \nu_{1}, \nu_{2}\right)=\lambda_{m}^{p}\left(\mu, \nu_{1}, \nu_{2}\right) \text {. }
$$

Proof. Let

$$
\widetilde{M}=\left\{u \in X: 1+\psi_{2}(u) \leq \psi_{1}(u)\right\}
$$

and denote by $\widetilde{\mathcal{K}}_{m}$ the family of nonempty, compact and symmetric subsets $K$ of $\widetilde{M}$ such that $i(K) \geq m$.

Of course, the topologies of $X$ and of $L_{l o c}^{p}\left(\mathbb{R}^{N}\right)$ agree on finite dimensional subspaces. Moreover, assumption (b) of Remark 3.6 is satisfied by $\varphi, \psi_{1}$ and $\psi_{2}$ in the space $X$, while assumption $(a)$ of Proposition 6.5 is just assumption $(i)$. Combining Theorem 3.5 with Proposition 6.5 , we infer that

$$
\inf _{K \in \widetilde{\mathcal{K}}_{m}} \max _{u \in K} \varphi(u)=\lambda_{m}^{p}\left(\mu, \nu_{1}, \nu_{2}\right)
$$

Of course, we have

$$
\inf _{K \in \widetilde{\mathcal{K}}_{m}} \max _{u \in K} \varphi(u) \leq \inf _{K \in \widehat{\mathcal{K}}_{m}} \max _{u \in K} \varphi(u)
$$

as $\widehat{M} \subseteq \widetilde{M}$. On the other hand, if $K \in \widetilde{\mathcal{K}}_{m}$, we have that

$$
\widehat{K}=\left\{\frac{u}{\left(\psi_{1}(u)-\psi_{2}(u)\right)^{1 / p}}: u \in K\right\}
$$

satisfies $\widehat{K} \in \widehat{\mathcal{K}}_{m}$ and $\max _{u \in \widehat{K}} \varphi(u) \leq \max _{u \in K} \varphi(u)$, whence

$$
\inf _{K \in \widehat{\mathcal{K}}_{m}} \max _{u \in K} \varphi(u) \leq \inf _{K \in \widetilde{\mathcal{K}}_{m}} \max _{u \in K} \varphi(u)
$$

and the assertion follows. 
Corollary 8.5. If there exists $u \in W_{c}^{1, p}\left(\mathbb{R}^{N}\right)$ such that

$$
\int|u|^{p} d \mu<+\infty, \quad \int|u|^{p} d \nu_{2}<\int|u|^{p} d \nu_{1}<+\infty, \quad \lim _{r \rightarrow 0} \int_{B_{r}(x)}|u|^{p} d \nu_{1}=0 \quad \text { for all } x \in \mathbb{R}^{N},
$$

then we have $\hat{\lambda}_{m}^{p}\left(\mu, \nu_{1}, \nu_{2}\right)<+\infty$ for all $m \geq 1$.

Proof. It follows from Proposition 6.3 and Theorem 8.4.

Theorem 8.6. The functionals $\varphi, \psi_{1}$ and $\psi_{2}$ satisfy the assumptions (ie) and (iie) of Section 4. In particular, the assertions of Theorem 4.5 hold true.

Proof. Since $\psi_{1}^{\prime}$ is the composition

$$
\begin{aligned}
& X \quad \longrightarrow L^{p}\left(\mathbb{R}^{N}, \nu_{1}\right) \longrightarrow L^{p^{\prime}}\left(\mathbb{R}^{N}, \nu_{1}\right) \longrightarrow X^{\prime} \\
& u \quad \mapsto \quad u \quad \mapsto \quad|u|^{p-2} u \quad \mapsto \quad \psi_{1}^{\prime}(u)
\end{aligned}
$$

the complete continuity of $\psi_{1}^{\prime}$ follows from the complete continuity of the first map and the continuity of the other maps.

Given $\lambda>0$, it is standard (see e.g.[6]) that $\left(\varphi^{\prime}+\lambda \psi_{2}^{\prime}+\lambda \psi_{1}^{\prime}\right)$ is of class $(S)_{+}$. Then also

$$
\varphi^{\prime}+\lambda \psi_{2}^{\prime}=\left(\varphi^{\prime}+\lambda \psi_{2}^{\prime}+\lambda \psi_{1}^{\prime}\right)-\lambda \psi_{1}^{\prime}
$$

is of class $(S)_{+}$.

Finally, if $u \in X \backslash\{0\}$ satisfies $\varphi(u)=0$, then $u$ is a nonzero constant, $p \geq N, \mu\left(\mathbb{R}^{N}\right)=0$ and we cannot have

by assumption (ii).

$$
\int|u|^{p} d \nu_{2} \leq \int|u|^{p} d \nu_{1}<+\infty
$$

Example 8.7. Let $N=1, p=2, \mu=0$ and

$$
\nu_{1}(B)=\mathcal{L}^{1}(B \cap] 0,1[), \quad \nu_{2}(B)=\mathcal{L}^{1}(B \cap]-1,0[), \quad \text { for all } B \in \mathcal{B}(\mathbb{R}) .
$$

Then we have

$$
\inf \left\{\varphi(u): \psi_{1}(u)-\psi_{2}(u)=1\right\}=0, \quad \varphi(u)>0 \text { for all } u \text { with } \psi_{1}(u)-\psi_{2}(u)>0 .
$$

On the other hand, assumption $(i i)$ is not satisfied.

\section{ON the EXISTENCE OF OPtimal CAPACITARY MEASURES}

Let $\underline{\mu}, \nu \in \mathcal{M}_{0}^{p}\left(\mathbb{R}^{N}\right)$ and $W: \mathbb{R}^{N} \rightarrow\left[0,+\infty\left[\right.\right.$ be a $\mathcal{L}^{N}$-measurable function. Let also $V_{\underline{\mu}+\nu}$ be the $\mathcal{L}^{N}$-measurable function introduced in Definition 5.8 and set $\nu_{1}=W \mathcal{L}^{N}, \nu_{2}=\nu$ and

$$
\lambda_{m}^{p}(\mu)=\lambda_{m}^{p}\left(\mu, \nu_{1}, \nu_{2}\right) \quad \text { for all } \mu \in \mathcal{M}_{0}^{p}\left(\mathbb{R}^{N}\right) \text { and } m \geq 1 .
$$

If $N \geq 2$, we define a convex function $\tau: \mathbb{R} \rightarrow \mathbb{R}$ by

$$
\tau(s)=\sum_{k=N}^{\infty} \frac{|s|^{\frac{k}{N-1}}}{k !}
$$

and denote by $\tau^{*}: \mathbb{R} \rightarrow \mathbb{R}$ its conjugate function, namely

$$
\tau^{*}(t)=\sup _{s \in \mathbb{R}}(t s-\tau(s)) .
$$

Since

we have

$$
\tau\left(2^{N-1} s\right) \geq 2^{N} \tau(s) \quad \text { for all } s \in \mathbb{R},
$$

Throughout this section, we assume that:

$(\mu W)$

- if $p<N$, we have

$$
\int_{\left\{V_{\underline{\mu}}+\nu<R W\right\}} W^{N / p} d \mathcal{L}^{N}<+\infty \quad \text { for all } R>0 ;
$$


- if $p=N$, we have

$$
\inf _{\varepsilon>0} \mathcal{L}^{N}\left(\left\{V_{\underline{\mu}+\nu}<\varepsilon\right\}\right)<+\infty
$$

and

$$
\int_{\left\{V_{\underline{\mu}+\nu}<R W\right\}} \tau^{*}(W) d \mathcal{L}^{N}<+\infty \quad \text { for all } R>0 ;
$$

- if $p>N$, we have

$$
\inf _{\varepsilon>0} \mathcal{L}^{N}\left(\left\{V_{\underline{\mu}+\nu}<\varepsilon\right\}\right)<+\infty
$$

and there exists $q \in[1, \infty[$ such that

$$
\int_{\left\{V_{\underline{\mu}}+\nu<R W\right\}} W^{q} d \mathcal{L}^{N}<+\infty \quad \text { for all } R>0 .
$$

Proposition 9.1. The following facts hold:

(a) if $\left(\mu^{(n)}\right)$ is locally $\gamma$-convergent to $\mu$ in $\mathcal{M}_{0}^{p}\left(\mathbb{R}^{N}\right)$ and $\mu^{(n)} \geq \underline{\mu}$ for all $n \in \mathbb{N}$, then $\mu \geq \underline{\mu}$ and

$$
\lambda_{m}^{p}(\mu) \leq \liminf _{n \rightarrow \infty} \lambda_{m}^{p}\left(\mu^{(n)}\right) \quad \text { for all } m \geq 1 ;
$$

(b) for every $\mu \in \mathcal{M}_{0}^{p}\left(\mathbb{R}^{N}\right)$ with $\mu \geq \underline{\mu}$, the assumptions ( $i$ ) and (ii) of Section 8 are satisfied by $\left(\mu, W \mathcal{L}^{N}, \nu\right)$, in particular

$$
\hat{\lambda}_{m}^{p}\left(\mu, W \mathcal{L}^{N}, \nu\right)=\lambda_{m}^{p}\left(\mu, W \mathcal{L}^{N}, \nu\right), \quad \text { for all } m \geq 1 ;
$$

(c) for every $\mu \in \mathcal{M}_{0}^{p}\left(\mathbb{R}^{N}\right)$, we have

$$
\lambda_{m}^{p}(\mu)<+\infty \quad \text { for all } m \geq 1
$$

if and only if there exists $u \in W_{c}^{1, p}\left(\mathbb{R}^{N}\right)$ such that

$$
\int|u|^{p} d \mu<+\infty, \quad \int|u|^{p} d \nu<\int|u|^{p} W d \mathcal{L}^{N}<+\infty .
$$

Proof.

(a) By Corollary 5.25 we have $\mu \geq \mu$. The second assertion follows from Theorem 7.3 as soon as the assumptions (is)-(iis)-(iiis) are verified. We deal first with assumption (is). Actually, we prove a stronger statement, which will be useful also in the verification of (iiis).

Let us consider a strictly increasing sequence $\left(n_{k}\right)$ in $\mathbb{N}$ and a sequence $\left(u^{(k)}\right)$ in $W_{c}^{1, p}\left(\mathbb{R}^{N}\right)$ converging in $L_{l o c}^{p}\left(\mathbb{R}^{N}\right)$ to $u \in W_{l o c}^{1, p}\left(\mathbb{R}^{N}\right)$ with

We claim that

$$
\sup _{k}\left(\int\left|\nabla u^{(k)}\right|^{p} d \mathcal{L}^{N}+\int\left|u^{(k)}\right|^{p} d \mu^{\left(n_{k}\right)}+\int\left|u^{(k)}\right|^{p} d \nu\right)<+\infty .
$$

$$
\limsup _{k \rightarrow \infty} \int\left|u^{(k)}\right|^{p} W d \mathcal{L}^{N} \leq \int|u|^{p} W d \mathcal{L}^{N}<+\infty .
$$

Up to a subsequence, $\left(u^{(k)}\right)$ is convergent to $u \mathcal{L}^{N}$-a.e. in $\mathbb{R}^{N}$ and we have

$$
\sup _{k}\left(\int\left|\nabla u^{(k)}\right|^{p} d \mathcal{L}^{N}+\int\left|u^{(k)}\right|^{p} d \underline{\mu}+\int\left|u^{(k)}\right|^{p} d \nu\right)<+\infty .
$$

Since for every $R>0$ it is

$$
\int_{\left\{R W \leq V_{\underline{\mu}+\nu}\right\}}\left|u^{(k)}\right|^{p} W d \mathcal{L}^{N} \leq \frac{1}{R}\left(\int\left|u^{(k)}\right|^{p} d \underline{\mu}+\int\left|u^{(k)}\right|^{p} d \nu\right),
$$

it is enough to show that

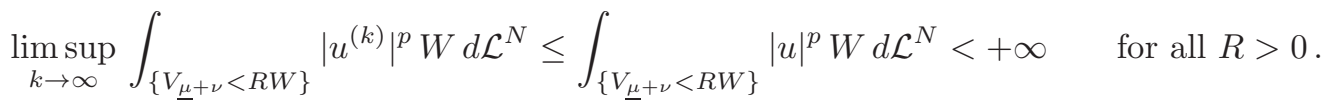

In the case $p<N$, the sequence $\left(u^{(k)}\right)$ is bounded in $L^{p^{*}}\left(\mathbb{R}^{N}\right)$, so that (9.2) follows from assumption $(\mu W)$.

If $p \geq N$, first of all by assumption $(\mu W)$ there exists $\varepsilon>0$ such that

$$
\mathcal{L}^{N}\left(\left\{V_{\underline{\mu}}+\nu<\varepsilon\right\}\right)<+\infty .
$$


If we set $C=\left\{V_{\underline{\mu}}+\nu \geq \varepsilon\right\}$, we have

$$
\sup _{k}\left(\int_{\mathbb{R}^{N}}\left|\nabla u^{(k)}\right|^{p} d \mathcal{L}^{N}+\varepsilon \int_{C}\left|u^{(k)}\right|^{p} d \mathcal{L}^{N}\right)<+\infty
$$

and $\mathcal{L}^{N}\left(\mathbb{R}^{N} \backslash C\right)<+\infty$. Since

$$
\sup _{k} \mathcal{L}^{N}\left(\left\{\left|u^{(k)}\right| \geq 1\right\}\right)<+\infty
$$

it follows that

$$
\sup _{k}\left(\int_{\mathbb{R}^{N}}\left|\nabla u^{(k)}\right|^{p} d \mathcal{L}^{N}+\int_{\mathbb{R}^{N}}\left|u^{(k)}\right|^{p} d \mathcal{L}^{N}\right)<+\infty .
$$

Now, in the case $p=N$, according to [35, Theorem 1.1] there exist $d_{N}, \alpha_{N}>0$ such that

$$
\int \tau\left(\alpha_{N}|v|^{N}\right) d \mathcal{L}^{N} \leq d_{N} \quad \text { whenever } v \in W_{c}^{1, N}\left(\mathbb{R}^{N}\right) \text { and } \int|\nabla v|^{N} d \mathcal{L}^{N}+\int|v|^{N} d \mathcal{L}^{N} \leq 1 .
$$

Therefore, for every $\sigma>0$ there exists $j \geq 1$ such that

$$
2^{-j} \int \tau\left(2^{-j}\left|u^{(k)}\right|^{N}\right) d \mathcal{L}^{N}<\sigma \quad \text { for all } k \in \mathbb{N} .
$$

On the other hand, we have

$$
\left|u^{(k)}\right|^{N} W \leq 2^{-j} \tau\left(2^{-j}\left|u^{(k)}\right|^{N}\right)+2^{-j} \tau^{*}\left(2^{2 j} W\right) \quad \text { a.e. in } \mathbb{R}^{N}
$$

and

$$
\int_{\left\{V_{\underline{\mu}+\nu}<R W\right\}} \tau^{*}\left(2^{2 j} W\right) d \mathcal{L}^{N}<+\infty \quad \text { for all } R, j
$$

by assumption $(\mu W)$ and (9.1). Therefore (9.2) follows.

In the case $p>N$, we have that $\left(u^{(k)}\right)$ is bounded in each $L^{r}\left(\mathbb{R}^{N}\right)$ with $p \leq r \leq \infty$ and (9.2) follows again from assumption $(\mu W)$. Therefore assumption (is) is satisfied.

Assumption (iiis) follows from the previous step, Theorem 5.24 and Proposition 5.16.

Finally, in the case $p \geq N$ also assumption (iis) is satisfied, as $\mathcal{L}^{N}(C)=+\infty$ implies that $(\underline{\mu}+\nu)\left(\mathbb{R}^{N}\right)=$ $+\infty$.

(b) We argue as in the previous step, noting that assumption $(i)$ is a special case of $(i s)$.

(c) The assertion follows from Proposition 6.3.

Theorem 9.2. Let $\Psi:[0,+\infty] \rightarrow[0,+\infty]$ be a function as in Corollary 5.26 and let

$$
0<c \leq \int \Psi\left(V_{\underline{\mu}}\right) d \mathcal{L}^{N} .
$$

Denote by $\mathcal{M}$ the set of $\mu$ 's in $\mathcal{M}_{0}^{p}\left(\mathbb{R}^{N}\right)$ such that

$$
\mu \geq \underline{\mu}, \quad \int \Psi\left(V_{\mu}\right) d \mathcal{L}^{N} \leq c
$$

and such that there exists $u \in W_{c}^{1, p}\left(\mathbb{R}^{N}\right)$ satisfying

$$
\int|u|^{p} d \mu<+\infty, \quad \int|u|^{p} d \nu<\int|u|^{p} W d \mathcal{L}^{N}<+\infty .
$$

If $\mathcal{M} \neq \emptyset$ then, for every $F: \mathbb{R}^{k} \rightarrow \mathbb{R}$ nondecreasing in each variable and lower semicontinuous, there exists a minimum $\mu \in \mathcal{M}$ of

$$
\left\{\mu \mapsto F\left(\lambda_{1}^{p}(\mu), \ldots, \lambda_{k}^{p}(\mu)\right)\right\}
$$

satisfying

$$
\int \Psi\left(V_{\mu}\right) d \mathcal{L}^{N}=c
$$


Proof. If

$$
\int \Psi\left(V_{\underline{\mu}}\right) d \mathcal{L}^{N}=c
$$

then $\underline{\mu} \in \mathcal{M}$ is a minimum with the required property. If

$$
\int \Psi\left(V_{\underline{\mu}}\right) d \mathcal{L}^{N}>c
$$

let $\mathbb{R}_{l s c}$ be the set $\mathbb{R}$ endowed with the topology of the lower semicontinuity: a subset $U$ of $\mathbb{R}$ is said to be open if $U=] s,+\infty$ [ for some $s \in \overline{\mathbb{R}}$.

For every $\mu \in \mathcal{M}$, we have $\lambda_{m}^{p}(\mu)<+\infty$ for all $m \geq 1$ by $(c)$ of Proposition 9.1 . Then the map

$$
\left\{\mu \mapsto\left(\lambda_{1}^{p}(\mu), \ldots, \lambda_{k}^{p}(\mu)\right)\right\}
$$

is continuous from $\mathcal{M}$ into $\mathbb{R}_{l s c}^{k}$ by Proposition 9.1 and the function $F$ is lower semicontinuous from $\mathbb{R}_{l s c}^{k}$ into $\mathbb{R}$. Therefore the functional

$$
\left\{\mu \mapsto F\left(\lambda_{1}^{p}(\mu), \ldots, \lambda_{k}^{p}(\mu)\right)\right\}
$$

is lower semicontinuous from $\mathcal{M}$ into $\mathbb{R}$.

Let $\bar{\mu} \in \mathcal{M}$. Since $F$ is nondecreasing in each variable, it is enough to restrict the minimization to

$$
\mathcal{N}=\left\{\mu \in \mathcal{M}: \lambda_{1}^{p}(\mu) \leq \lambda_{k}^{p}(\bar{\mu})\right\} .
$$

Observe that, if $\left(\mu^{(n)}\right)$ is a sequence in $\mathcal{N}$ locally $\gamma$-converging to $\mu$ in $\mathcal{M}_{0}^{p}\left(\mathbb{R}^{N}\right)$, then $\lambda_{1}^{p}(\mu)<+\infty$ by $(a)$ of Proposition 9.1, which implies that there exists $u \in W_{c}^{1, p}\left(\mathbb{R}^{N}\right)$ satisfying

$$
\int|u|^{p} d \mu<+\infty, \quad \int|u|^{p} d \nu<\int|u|^{p} W d \mathcal{L}^{N}<+\infty
$$

by (a) of Proposition 6.3. Combining this fact with Corollary 5.26 and $(a)$ of Proposition 9.1, it follows that $\mu \in \mathcal{N}$, so that $\mathcal{N}$ is a nonempty and closed subset of the metrizable and compact space $\mathcal{M}_{0}^{p}\left(\mathbb{R}^{N}\right)$.

Therefore, there exists a minimum $\mu \in \mathcal{M}$ of

$$
\left\{\mu \mapsto F\left(\lambda_{1}^{p}(\mu), \ldots, \lambda_{k}^{p}(\mu)\right)\right\} .
$$

If

$$
\int \Psi\left(V_{\mu}\right) d \mathcal{L}^{N}<c
$$

define for $t \geq 0$

$$
\begin{aligned}
& V^{(t)}=\Psi^{-1}\left[\min \left\{\Psi\left(V_{\underline{\mu}}\right), \Psi\left(V_{\mu}\right)+t \exp \left(-|x|^{2}\right)\right\}\right], \\
& \mu^{(t)}=\infty_{\mathbb{R}^{N} \backslash A_{\underline{\mu}}}+\underline{\mu}_{s}+V^{(t)} \mathcal{L}^{N} .
\end{aligned}
$$

Then $V_{\underline{\mu}} \leq V^{(t)} \leq V_{\mu} \mathcal{L}^{N}$-a.e. in $\mathbb{R}^{N}$ and $\underline{\mu} \leq \mu^{(t)} \leq \mu$. Moreover, from $(b)$ and $(d)$ of Proposition 5.9 we infer that

$$
V_{\mu^{(t)}} \geq V^{(t)} \quad \mathcal{L}^{N} \text {-a.e. in } \mathbb{R}^{N} .
$$

In the case $t=0$, we have $V^{(0)}=V_{\mu}$ and $V_{\mu} \mathcal{L}^{N}$ is $\sigma$-finite on $A_{\mu}$, whence $V_{\mu}(0)=V^{(0)} \mathcal{L}^{N}$-a.e. in $\mathbb{R}^{N}$. If $t>0$, let

Then we have

$$
R(t, n)=\Psi^{-1}\left(\Psi(+\infty)+t \exp \left(-n^{2}\right)\right)
$$

$$
V^{(t)} \leq \max \left\{V_{\underline{\mu}}, R(t, n)\right\} \quad \mathcal{L}^{N} \text {-a.e. in } B_{n}(0)
$$

whence $A_{\mu^{(t)}}=A_{\underline{\mu}}$. It follows that

$$
V_{\mu^{(t)}}=V^{(t)} \quad \mathcal{L}^{N} \text {-a.e. in } \mathbb{R}^{N}, \text { for all } t \geq 0 .
$$

If we choose $t>0$ such that

$$
\int \Psi\left(V_{\mu^{(t)}}\right) d \mathcal{L}^{N}=c,
$$

then $\mu^{(t)}$ is a minimum with the required property. 
Theorem 9.3. Let

$$
0<c \leq \mathcal{L}^{N}\left(A_{\underline{\mu}}\right)
$$

and denote by $\mathcal{M}$ the set of $\mu$ 's in $\mathcal{M}_{0}^{p}\left(\mathbb{R}^{N}\right)$ such that

$$
\mu \geq \underline{\mu}, \quad \mathcal{L}^{N}\left(A_{\mu}\right) \leq c
$$

and such that there exists $u \in W_{c}^{1, p}\left(\mathbb{R}^{N}\right)$ satisfying

$$
\int|u|^{p} d \mu<+\infty, \quad \int|u|^{p} d \nu<\int|u|^{p} W d \mathcal{L}^{N}<+\infty .
$$

If $\mathcal{M} \neq \emptyset$ then, for every $F: \mathbb{R}^{k} \rightarrow \mathbb{R}$ nondecreasing in each variable and lower semicontinuous, there exists a minimum $\mu \in \mathcal{M}$ of

$$
\left\{\mu \mapsto F\left(\lambda_{1}^{p}(\mu), \ldots, \lambda_{k}^{p}(\mu)\right)\right\}
$$

satisfying

$$
\mathcal{L}^{N}\left(A_{\mu}\right)=c .
$$

Proof. If $\mathcal{L}^{N}\left(A_{\underline{\mu}}\right)=c$, then $\underline{\mu}$ is a minimum with the required property. Otherwise, assume that $\mathcal{L}^{N}\left(A_{\underline{\mu}}\right)>c$. Arguing as in the proof of Theorem 9.2, we find a minimum $\mu \in \mathcal{M}$ of

$$
\left\{\mu \mapsto F\left(\lambda_{1}^{p}(\mu), \ldots, \lambda_{k}^{p}(\mu)\right)\right\} .
$$

If $\mathcal{L}^{N}\left(A_{\mu}\right)<c$, consider $\mu^{(r)} \in \mathcal{M}_{0}^{p}\left(\mathbb{R}^{N}\right)$ defined by

$$
\mu^{(r)}(B)=\underline{\mu}\left(B \cap B_{r}(0)\right)+\tilde{\mu}\left(B \backslash B_{r}(0)\right) \quad \text { for all } B \in \mathcal{B}\left(\mathbb{R}^{N}\right),
$$

where $\tilde{\mu}$ is the outer regular representative of $\mu$ given by Proposition 5.3. Then it is easily seen that $\underline{\mu} \leq \mu^{(r)} \leq \mu$ and that

$$
A_{\mu} \cup\left(A_{\underline{\mu}} \cap B_{r}(0)\right) \subseteq A_{\mu}^{(r)} .
$$

If $\mu^{(r)}(W)<+\infty$ for some Borel and $p$-finely open $W$, there exists a Borel and $p$-quasi open $A$ such that $W \backslash B_{r}(0) \subseteq A$ and $\mu(A)<+\infty$, so that

$$
W \cap B_{r}(0) \subseteq A_{\underline{\mu}} \cap B_{r}(0), \quad \operatorname{cap}_{p}\left[\left(W \backslash B_{r}(0)\right) \backslash A_{\mu}\right] \leq \operatorname{cap}_{p}\left(A \backslash A_{\mu}\right)=0
$$

by $(a)$ of Proposition 5.7. From the quasi-Lindelöf property we infer that

$$
\operatorname{cap}_{p}\left[A_{\mu^{(r)}} \backslash\left(A_{\mu} \cup\left(A_{\underline{\mu}} \cap B_{r}(0)\right)\right)\right]=0,
$$

whence

$$
\mathcal{L}^{N}\left(A_{\mu(r)}\right)=\mathcal{L}^{N}\left(A_{\mu} \cup\left(A_{\underline{\mu}} \cap B_{r}(0)\right)\right) .
$$

If we choose $r>0$ such that

$$
\mathcal{L}^{N}\left(A_{\mu(r)}\right)=c,
$$

then $\mu^{(r)}$ is a minimum with the required property.

Now we first consider the particular case in which

$$
\underline{\mu}(B)=\left\{\begin{array}{ll}
\int_{B \cap \underline{A}} \underline{V} d \mathcal{L}^{N} & \text { if } \operatorname{cap}_{p}(B \backslash \underline{A})=0, \\
+\infty & \text { if } \operatorname{cap}_{p}(B \backslash \underline{A})>0,
\end{array} \quad \text { for all } B \in \mathcal{B}\left(\mathbb{R}^{N}\right) .\right.
$$

for some $p$-quasi open subset $\underline{A}$ of $\mathbb{R}^{N}$ and some $p$-quasi upper semicontinuous function $\underline{V}: \underline{A} \rightarrow[0,+\infty]$.

Corollary 9.4. Let $\Psi:[0,+\infty] \rightarrow[0,+\infty]$ be a function as in Corollary 5.26 and let

$$
0<c \leq \int_{\underline{A}} \Psi(\underline{V}) d \mathcal{L}^{N} .
$$

Denote by $\mathcal{V}$ the set of $\mathcal{L}^{N}$-measurable functions $V: \underline{A} \rightarrow[0,+\infty]$ such that

$$
V \geq \underline{V} \quad \mathcal{L}^{N} \text {-a.e. in } \underline{A}, \quad \int_{\underline{A}} \Psi(V) d \mathcal{L}^{N} \leq c
$$


and such that there exists $u \in W_{0}^{1, p}(\underline{A})$ satisfying

$$
\int_{\underline{A}}|u|^{p} V d \mathcal{L}^{N}<+\infty, \quad \int_{\underline{A}}|u|^{p} d \nu<\int_{\underline{A}}|u|^{p} W d \mathcal{L}^{N}<+\infty .
$$

If $\mathcal{V} \neq \emptyset$ then, for every $F: \mathbb{R}^{k} \rightarrow \mathbb{R}$ nondecreasing in each variable and lower semicontinuous, there exists a minimum $V \in \mathcal{V}$ of

$$
\left\{V \mapsto F\left(\lambda_{1}^{p}(V), \ldots, \lambda_{k}^{p}(V)\right)\right\}
$$

satisfying

$$
\int_{\underline{A}} \Psi(V) d \mathcal{L}^{N}=c
$$

where $\lambda_{m}^{p}(V)=\lambda_{m}^{p}(\mu)$ with

$$
\mu(B)=\left\{\begin{array}{ll}
\int_{B \cap \underline{A}} V d \mathcal{L}^{N} & \text { if } \operatorname{cap}_{p}(B \backslash \underline{A})=0, \\
+\infty & \text { if } \operatorname{cap}_{p}(B \backslash \underline{A})>0,
\end{array} \quad \text { for all } B \in \mathcal{B}\left(\mathbb{R}^{N}\right) .\right.
$$

Proof. We aim to apply Theorem 9.2. Without loss of generality, we may assume that $\Psi(+\infty)=0$. Consider $\underline{V}$ and each $V$ defined on all $\mathbb{R}^{N}$ with value $+\infty$ outside $\underline{A}$. Then the definition of $\underline{\mu}$ and $(9.3)$ can be reformulated as

$$
\underline{\mu}=\infty_{\mathbb{R}^{N} \backslash \underline{A}}+\underline{V} \mathcal{L}^{N}, \quad \mu=\infty_{\mathbb{R}^{N} \backslash \underline{A}}+V \mathcal{L}^{N} .
$$

For every $u \in W_{0}^{1, p}(\underline{A})$ there exists a sequence $\left(u_{n}\right)$ in $W_{c}^{1, p}\left(\mathbb{R}^{N}\right)$ converging to $u$ in $W^{1, p}\left(\mathbb{R}^{N}\right)$ with $\left|u_{n}\right| \leq|u|$ q.e. in $\mathbb{R}^{N}$. Combining this fact with Proposition 5.9, we see that, if $V \in \mathcal{V}$ and $\mu$ is defined according to (9.3), then we have $\mu \in \mathcal{M}$. On the other hand, if $\mu \in \mathcal{M}$ we infer again from Proposition 5.9 that $V_{\mu} \in \mathcal{V}$. Moreover, by $(e)$ of Proposition 5.9 we have

$$
c \leq \int \Psi\left(V_{\underline{\mu}}\right) d \mathcal{L}^{N} .
$$

Let $\mu \in \mathcal{M}$ be a minimum of

$$
\left\{\mu \mapsto F\left(\lambda_{1}^{p}(\mu), \ldots, \lambda_{k}^{p}(\mu)\right)\right\}
$$

according to Theorem 9.2. By Proposition 5.9, since $F$ is nondecreasing in each variable, we have

$$
F\left(\lambda_{1}^{p}\left(V_{\mu}\right), \ldots, \lambda_{k}^{p}\left(V_{\mu}\right)\right) \leq F\left(\lambda_{1}^{p}(\mu), \ldots, \lambda_{k}^{p}(\mu)\right) \leq F\left(\lambda_{1}^{p}(V), \ldots, \lambda_{k}^{p}(V)\right) \text { for all } V \in \mathcal{V} .
$$

Since $V_{\mu} \in \mathcal{V}$, the assertion follows.

Then let us consider the particular case in which $\underline{\mu}=\infty_{\mathbb{R}^{N} \backslash \underline{A}}$ for some $p$-quasi open subset $\underline{A}$ of $\mathbb{R}^{N}$. For every $p$-quasi open subset $A$ of $\mathbb{R}^{N}$ and $m \geq 1$, we set $\lambda_{m}^{p}(\bar{A})=\lambda_{m}^{p}\left(\infty_{\mathbb{R}^{N} \backslash A}\right)$.

In this case assumption $(\mu W)$ reads:

- if $p<N$, we have

$$
\int_{\underline{A} \cap\left\{V_{\nu}<R W\right\}} W^{N / p} d \mathcal{L}^{N}<+\infty \quad \text { for all } R>0 ;
$$

- if $p=N$, we have

$$
\inf _{\varepsilon>0} \mathcal{L}^{N}\left(\underline{A} \cap\left\{V_{\nu}<\varepsilon\right\}\right)<+\infty
$$

and

$$
\int_{\underline{A} \cap\left\{V_{\nu}<R W\right\}} \tau^{*}(W) d \mathcal{L}^{N}<+\infty \quad \text { for all } R>0 ;
$$

- if $p>N$, we have

$$
\inf _{\varepsilon>0} \mathcal{L}^{N}\left(\underline{A} \cap\left\{V_{\nu}<\varepsilon\right\}\right)<+\infty
$$

and there exists $q \in[1, \infty[$ such that

$$
\int_{\underline{A} \cap\left\{V_{\nu}<R W\right\}} W^{q} d \mathcal{L}^{N}<+\infty \quad \text { for all } R>0 .
$$

In particular, assumption $(A W)$ is satisfied if $\mathcal{L}^{N}(\underline{A})<+\infty$ and $W=1$.

Corollary 9.5. The following facts hold: 
(a) if $\left(A^{(n)}\right)$ is a sequence of $p$-quasi open subsets of $\underline{A}$ locally $\gamma$-converging to a p-quasi open subset $A$, then $\operatorname{cap}_{p}(A \backslash \underline{A})=0$ and

$$
\lambda_{m}^{p}(A) \leq \liminf _{n \rightarrow \infty} \lambda_{m}^{p}\left(A^{(n)}\right) \quad \text { for all } m \geq 1 ;
$$

(b) for every p-quasi open subset $A$ of $\underline{A}$, the assumptions (i) and (ii) of Section 8 are satisfied by $\left(\infty_{\mathbb{R}^{N} \backslash A}, W \mathcal{L}^{N}, \nu\right)$, in particular

$$
\lambda_{m}^{p}(A)=\lambda_{m}^{p}\left(\infty_{\mathbb{R}^{N} \backslash A}, W \mathcal{L}^{N}, \nu\right)=\hat{\lambda}_{m}^{p}\left(\infty_{\mathbb{R}^{N} \backslash A}, W \mathcal{L}^{N}, \nu\right), \quad \text { for all } m \geq 1 ;
$$

(c) for every p-quasi open subset $A$ of $\mathbb{R}^{N}$, we have

$$
\lambda_{m}^{p}(A)<+\infty \quad \text { for all } m \geq 1
$$

if and only if there exists $u \in W_{0}^{1, p}(A)$ satisfying

$$
\int|u|^{p} d \nu<\int|u|^{p} W d \mathcal{L}^{N}<+\infty ;
$$

in particular, if $W=1, \nu=0$ and $0<\mathcal{L}^{N}(A)<+\infty$, then the eigenvalues $\lambda_{m}^{p}(A)$ agree with those defined by (1.1).

Proof. Taking into account Definition 5.15 and Proposition 5.9, it is a particular case of Proposition 9.1.

Corollary 9.6. Let $\left.c \in] 0, \mathcal{L}^{N}(\underline{A})\right]$ and denote by $\mathcal{A}$ the family of p-quasi open subsets $A$ of $\mathbb{R}^{N}$ such that

$$
A \subseteq \underline{A}, \quad \mathcal{L}^{N}(A) \leq c
$$

and such that there exists $u \in W_{0}^{1, p}(A)$ satisfying

$$
\int|u|^{p} d \nu<\int|u|^{p} W d \mathcal{L}^{N}<+\infty .
$$

If $\mathcal{A} \neq \emptyset$ then, for every $F: \mathbb{R}^{k} \rightarrow \mathbb{R}$ nondecreasing in each variable and lower semicontinuous, there exists a minimum in $\mathcal{A}$ of

$$
\left\{A \mapsto F\left(\lambda_{1}^{p}(A), \ldots, \lambda_{k}^{p}(A)\right)\right\}
$$

satisfying

$$
\mathcal{L}^{N}(A)=c .
$$

Proof. We aim to apply Theorem 9.3. By Proposition 5.9, if $A \in \mathcal{A}$ we have $\infty_{\mathbb{R}^{N} \backslash A} \in \mathcal{M}$. On the other hand, if $\mu \in \mathcal{M}$ we infer again from Proposition 5.9 that $\operatorname{cap}_{p}\left(A_{\mu} \backslash \underline{A}\right)=0$, whence $A_{\mu} \cap \underline{A} \in \mathcal{A}$.

Let $\mu \in \mathcal{M}$ be a minimum of

$$
\left\{\mu \mapsto F\left(\lambda_{1}^{p}(\mu), \ldots, \lambda_{k}^{p}(\mu)\right)\right\}
$$

according to Theorem 9.3. By Proposition 5.9, since $F$ is nondecreasing in each variable, we have

$$
F\left(\lambda_{1}^{p}\left(A_{\mu} \cap \underline{A}\right), \ldots, \lambda_{k}^{p}\left(A_{\mu} \cap \underline{A}\right)\right) \leq F\left(\lambda_{1}^{p}(\mu), \ldots, \lambda_{k}^{p}(\mu)\right) \leq F\left(\lambda_{1}^{p}(A), \ldots, \lambda_{k}^{p}(A)\right) \quad \text { for all } A \in \mathcal{A} .
$$

Since $A_{\mu} \cap \underline{A} \in \mathcal{A}$, the assertion follows.

Proof of Theorem 1.3. Let $\underline{A}=\Omega, W=1$ and $\nu=0$, so that assumption $(A W)$ is satisfied. According to Corollary 9.5, for every $p$-quasi open subset $A$ of $\Omega$ with $\mathcal{L}^{N}(A)>0$, the eigenvalues $\lambda_{m}^{p}(A)$ agree with those defined by (1.1). Then the assertion follows from Corollary 9.6.

Proof of Theorem 1.4. We aim to apply Corollary 9.4. Without loss of generality, we may assume that $\nu \in \mathcal{M}_{0}^{p}\left(\mathbb{R}^{N}\right)$. Let $\underline{A}=\Omega, \underline{V}=0$ and $W=1$, so that $\underline{\mu}=\infty_{\mathbb{R}^{N} \backslash \underline{A}}$. A fortiori we have $V_{\underline{\mu}+\nu}=+\infty$ on $\mathbb{R}^{N} \backslash \Omega$. Since $\Omega$ has finite measure, it is easily seen that assumption $(\mu W)$ is satisfied. Then the assertion follows. 


\section{REFERENCES}

[1] L. Ambrosio, N. Fusco, and D. Pallara, Functions of bounded variation and free discontinuity problems, Oxford Mathematical Monographs, The Clarendon Press, Oxford University Press, New York, 2000.

[2] L. Ambrosio And P. Tilli, Topics on analysis in metric spaces, vol. 25 of Oxford Lecture Series in Mathematics and its Applications, Oxford University Press, Oxford, 2004.

[3] P. R. S. Antunes, Extremal p-Laplacian eigenvalues, Nonlinearity, 32 (2019), pp. 5087-5109.

[4] T. BARTSCH, Topological methods for variational problems with symmetries, vol. 1560 of Lecture Notes in Mathematics, Springer-Verlag, Berlin, 1993.

[5] M. Brelot, On topologies and boundaries in potential theory, Enlarged edition of a course of lectures delivered in 1966. Lecture Notes in Mathematics, Vol. 175, Springer-Verlag, Berlin-New York, 1971.

[6] F. E. Browder, Fixed point theory and nonlinear problems, Bull. Amer. Math. Soc. (N.S.), 9 (1983), pp. 1-39.

[7] D. Bucur, Uniform concentration-compactness for Sobolev spaces on variable domains, J. Differential Equations, 162 (2000), pp. 427-450.

[8] - Minimization of the k-th eigenvalue of the Dirichlet Laplacian, Arch. Ration. Mech. Anal., 206 (2012), pp. 10731083.

[9] D. Bucur and G. Buttazzo, Variational methods in shape optimization problems, Progress in Nonlinear Differential Equations and their Applications, 65, Birkhäuser Boston, Inc., Boston, MA, 2005.

[10] D. Bucur and D. Mazzoleni, A surgery result for the spectrum of the Dirichlet Laplacian, SIAM J. Math. Anal., 47 (2015), pp. 4451-4466.

[11] G. Buttazzo and G. Dal Maso, An existence result for a class of shape optimization problems, Arch. Rational Mech. Anal., 122 (1993), pp. 183-195.

[12] G. Buttazzo, A. Gerolin, B. Ruffini, and B. Velichkov, Optimal potentials for Schrödinger operators, J. Éc. polytech. Math., 1 (2014), pp. 71-100.

[13] R. S. Cantrell and C. Cosner, Spatial ecology via reaction-diffusion equations, Wiley Series in Mathematical and Computational Biology, John Wiley \& Sons, Ltd., Chichester, 2003.

[14] T. Champion and L. De Pascale, Asymptotic behaviour of nonlinear eigenvalue problems involving p-Laplacian-type operators, Proc. Roy. Soc. Edinburgh Sect. A, 137 (2007), pp. 1179-1195.

[15] S. Cingolani, M. Degiovanni, And G. Vannella, Amann-Zehnder type results for p-Laplace problems, Ann. Mat. Pura Appl. (4), 197 (2018), pp. 605-640.

[16] J.-N. Corvellec, M. Degiovanni, and M. Marzocchi, Deformation properties for continuous functionals and critical point theory, Topol. Methods Nonlinear Anal., 1 (1993), pp. 151-171.

[17] G. Dal Maso, Г-convergence and $\mu$-capacities, Ann. Scuola Norm. Sup. Pisa Cl. Sci. (4), 14 (1987), pp. $423-464$.

[18] — An introduction to Г-convergence, vol. 8 of Progress in Nonlinear Differential Equations and their Applications, Birkhäuser Boston, Inc., Boston, MA, 1993.

[19] G. Dal Maso and U. Mosco, Wiener's criterion and $\Gamma$-convergence, Appl. Math. Optim., 15 (1987), pp. 15-63.

[20] G. Dal Maso and F. Murat, Asymptotic behaviour and correctors for Dirichlet problems in perforated domains with homogeneous monotone operators, Ann. Scuola Norm. Sup. Pisa Cl. Sci. (4), 24 (1997), pp. 239-290.

[21] M. Degiovanni and S. Lancelotti, Linking over cones and nontrivial solutions for p-Laplace equations with $p$ superlinear nonlinearity, Ann. Inst. H. Poincaré Anal. Non Linéaire, 24 (2007), pp. 907-919.

[22] M. Degiovanni and M. Marzocchi, Limit of minimax values under $\Gamma$-convergence, Electron. J. Differential Equations, No. 266 (2014), p. 19.

[23] K. Deimling, Nonlinear functional analysis, Springer-Verlag, Berlin, 1985.

[24] I. Ekeland and R. TÉmam, Convex analysis and variational problems, vol. 28 of Classics in Applied Mathematics, Society for Industrial and Applied Mathematics (SIAM), Philadelphia, PA, english ed., 1999. Translated from the French.

[25] E. R. Fadell and P. H. Rabinowitz, Bifurcation for odd potential operators and an alternative topological index, J. Functional Analysis, 26 (1977), pp. 48-67.

[26] - Generalized cohomological index theories for Lie group actions with an application to bifurcation questions for Hamiltonian systems, Invent. Math., 45 (1978), pp. 139-174.

[27] N. Fusco, S. Mukherjee, And Y. R.-Y. Zhang, A variational characterisation of the second eigenvalue of the p-Laplacian on quasi open sets, Proc. Lond. Math. Soc. (3), 119 (2019), pp. 579-612.

[28] J. P. García Azorero and I. Peral Alonso, Existence and nonuniqueness for the p-Laplacian: nonlinear eigenvalues, Comm. Partial Differential Equations, 12 (1987), pp. 1389-1430.

[29] J. Heinonen, T. Kilpeläinen, And J. MalÝ, Connectedness in fine topologies, Ann. Acad. Sci. Fenn. Ser. A I Math., 15 (1990), pp. 107-123.

[30] J. Heinonen, T. Kilpeläinen, and O. Martio, Nonlinear potential theory of degenerate elliptic equations, Dover Publications, Inc., Mineola, NY, 2006. Unabridged republication of the 1993 original.

[31] A. Henrot, ed., Shape optimization and spectral theory, De Gruyter Open, Warsaw, 2017.

[32] A. D. IofFe, On lower semicontinuity of integral functionals. I, SIAM J. Control Optimization, 15 (1977), pp. 521-538.

[33] T. KilpeläInen and J. Malý, Supersolutions to degenerate elliptic equation on quasi open sets, Comm. Partial Differential Equations, 17 (1992), pp. 371-405.

[34] M. A. Krasnosel'skiI, Topological methods in the theory of nonlinear integral equations, Translated by A. H. Armstrong; translation edited by J. Burlak. A Pergamon Press Book, The Macmillan Co., New York, 1964. 
[35] Y. Li AND B. Ruf, A sharp Trudinger-Moser type inequality for unbounded domains in $\mathbb{R}^{n}$, Indiana Univ. Math. J., 57 (2008), pp. 451-480.

[36] D. Mazzoleni And A. Pratelli, Existence of minimizers for spectral problems, J. Math. Pures Appl. (9), 100 (2013), pp. $433-453$.

[37] P. H. Rabinowitz, Minimax methods in critical point theory with applications to differential equations, vol. 65 of CBMS Regional Conference Series in Mathematics, Published for the Conference Board of the Mathematical Sciences, Washington, DC; by the American Mathematical Society, Providence, RI, 1986.

[38] A. Szulkin, Ljusternik-Schnirelmann theory on $C^{1}$-manifolds, Ann. Inst. H. Poincaré Anal. Non Linéaire, 5 (1988), pp. 119-139.

[39] A. Szulkin And M. Willem, Eigenvalue problems with indefinite weight, Studia Math., 135 (1999), pp. $191-201$.

Dipartimento di Matematica e Fisica, Università Cattolica del Sacro Cuore, Via Trieste 17, 25121 Brescia, ITALY

Email address: marco.degiovanni@unicatt.it

Dipartimento di Matematica F. Casorati, Università di Pavia, Via Ferrata 5, 27100 Pavia, Italy

Email address: dario.mazzoleni@unipv.it 